\title{
VERIFICATION AND VALIDATION OF TMAP4
}

\author{
G. R. Longhurst \\ S. L. Harms \\ E. S. Marwil \\ B. G. Miller
}

July 8,1992

Idaho National Engineering Laboratory

P.O. Box 1625

Idaho Falls, ID 83415

Prepared for the

U.S. Department of Energy Office of Energy Research Under DOE Idaho Field Office Contract DE-AC07-76ID01570

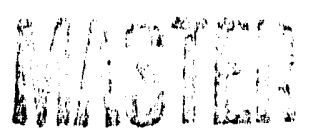




\begin{abstract}
The Tritium Migration Analysis Program MOD1/CY04 (TMAP4) was written to be used in analyzing experiments and for safety calculations that involve the injection, solution, diffusion, trapping, release, and other related processes experienced by hydrogen isotopes in materials. Because of the desire to make it suitable for analyzing safety issues, it is important that TMAP4 be certified (verified and validated) at Quality Assurance Level A. This report documents the work done to achieve that certification. The process includes assuring that the developed code meets the software requirements specified in the Software Quality Assurance Plan, verifying that the code functions in accordance with the written description and that it is self-consistent and internally correct, and validating that its computed results are in agreement with experimental data and/or known analytical solutions. Quality Level A certification for TMAP4 is specifically for implementation on an IBM PS/2 Model 70 operating under DOS 5.0. Certification for any other environment will require demonstration that all of the verification and validation tests documented here give the sarne results in the new environment.
\end{abstract}




\section{CONTENTS}

ABSTRACT $\ldots \ldots \ldots \ldots \ldots \ldots \ldots \ldots \ldots \ldots \ldots \ldots \ldots \ldots \ldots \ldots \ldots$ ii

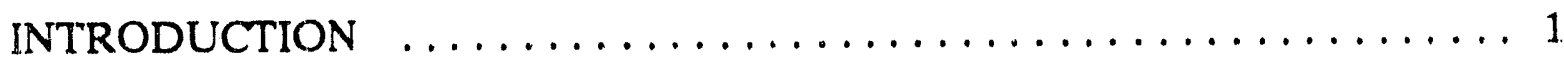

VERIFICATION $\ldots \ldots \ldots \ldots \ldots \ldots \ldots \ldots \ldots \ldots \ldots \ldots \ldots, \ldots \ldots \ldots$

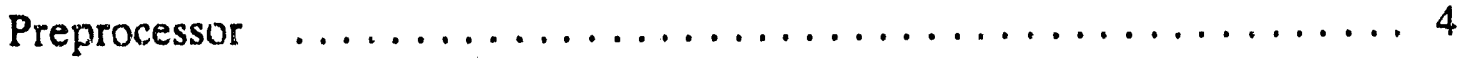

Computational Module Analysis $\ldots \ldots \ldots \ldots \ldots \ldots \ldots \ldots \ldots \ldots, \ldots \ldots \ldots$

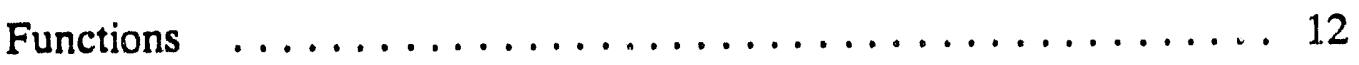

Common Block Irregularities $\ldots \ldots \ldots \ldots \ldots \ldots \ldots \ldots \ldots, \ldots \ldots \ldots$

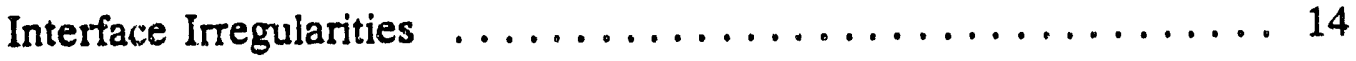

FORTRAN Extensions $\ldots \ldots \ldots \ldots \ldots \ldots \ldots \ldots \ldots \ldots \ldots \ldots$

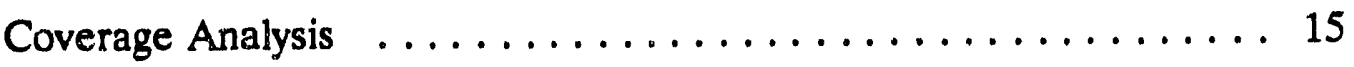

Complexity Analysis $\ldots \ldots \ldots \ldots \ldots \ldots \ldots \ldots \ldots \ldots . \ldots 19$

VALIDATION $\ldots \ldots \ldots \ldots \ldots \ldots \ldots \ldots \ldots \ldots \ldots \ldots \ldots \ldots \ldots \ldots, 22$

Comparison with Analytic Solutions $\ldots \ldots \ldots \ldots \ldots \ldots \ldots \ldots . \ldots 22$

1a) Depleting Source Problem $\ldots \ldots \ldots \ldots \ldots \ldots \ldots \ldots 22$

1b) Diffusion Problem with Constant Source Boundary Condition . 25

1c) Diffusion Problem with Partially Preloaded Slab $\ldots \ldots \ldots . .30$

1d) Permeation Problem with Trapping $\ldots \ldots \ldots \ldots \ldots \ldots \ldots, 33$

1e) Diffusion Problem with Composite Material Layers . . . . . 37

1f) Heat Sink/Source Problem $\ldots \ldots \ldots \ldots \ldots \ldots \ldots . \ldots 40$

1g) Simple Chemical Reaction Problem $\ldots \ldots \ldots \ldots \ldots \ldots, 41$

1h) System (Multiple Enclosure Volumes) Problem ......... 45

Comparison with Experiments $\ldots \ldots \ldots \ldots \ldots \ldots \ldots \ldots \ldots . \ldots 49$

2a) Ion Inplantation Experiment $\ldots \ldots \ldots \ldots \ldots \ldots \ldots \ldots 49$

2b) Material Diffusion Experiment $\ldots \ldots \ldots \ldots \ldots \ldots \ldots, \ldots$

2c) Test Cell Release Experiment $\ldots \ldots \ldots \ldots \ldots \ldots \ldots \ldots 54$

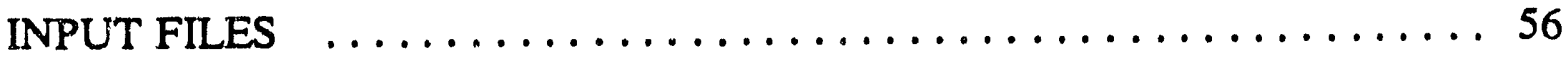

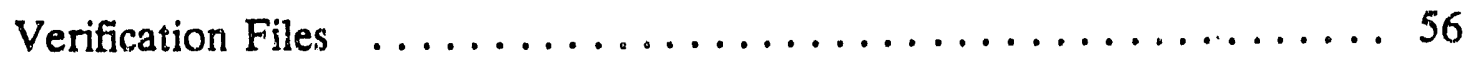

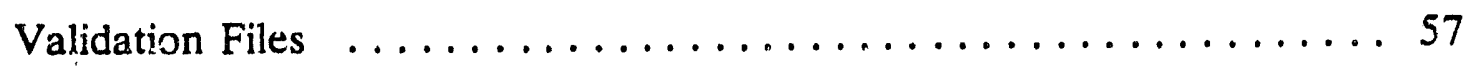




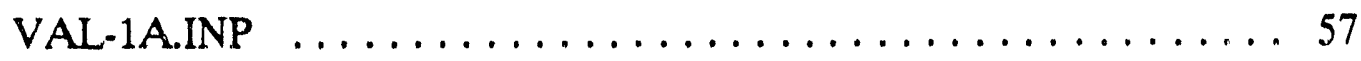

VAL-1B.INP $\ldots \ldots \ldots \ldots \ldots \ldots \ldots \ldots \ldots \ldots \ldots \ldots . \ldots \ldots$

VAL-1C.INP $\ldots \ldots \ldots \ldots \ldots \ldots \ldots \ldots \ldots \ldots \ldots \ldots \ldots \ldots \ldots \ldots$

VAL-1DA.INP $\ldots \ldots \ldots \ldots \ldots \ldots \ldots \ldots \ldots \ldots \ldots, 63$

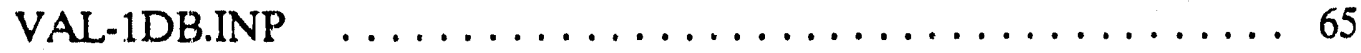

VAL-1E.INP $\ldots \ldots \ldots \ldots \ldots \ldots \ldots \ldots \ldots \ldots \ldots \ldots \ldots$

VAL-1FA.INP $\ldots \ldots \ldots \ldots \ldots \ldots \ldots \ldots \ldots \ldots \ldots \ldots$

VAL-1FB.INP $\ldots \ldots \ldots \ldots \ldots \ldots \ldots \ldots \ldots \ldots \ldots \ldots \ldots \ldots$

VAL-1GA.INP $\ldots \ldots \ldots \ldots \ldots \ldots \ldots \ldots \ldots \ldots \ldots \ldots$

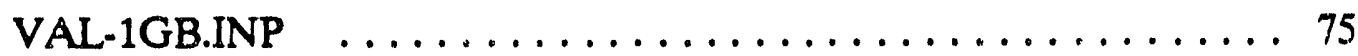

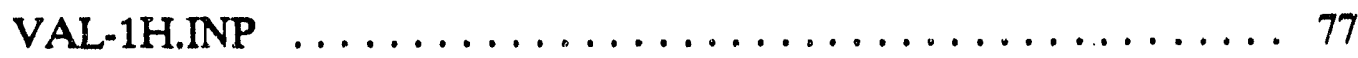

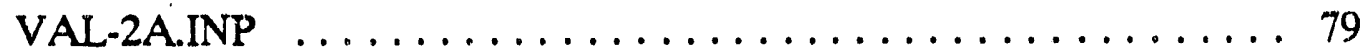

VAL-2BA.INP $\ldots \ldots \ldots \ldots \ldots \ldots \ldots \ldots \ldots \ldots \ldots, 81$

VAL-2BB.INP $\ldots \ldots \ldots \ldots \ldots \ldots \ldots \ldots \ldots \ldots \ldots \ldots$

REFERENCES $\ldots \ldots \ldots \ldots \ldots \ldots \ldots \ldots \ldots \ldots \ldots \ldots \ldots \ldots$

\section{FIGURES}

Figure 1 TMAP4 gives excellent results in comparison with the analytical solution for the depleting source problem.

Figure 2 Concentration profile at $\mathrm{t}=25 \mathrm{~s}$ for semi-infinite slab with constant source.

Figure 3 Comparison of TMAP4 calculation with the analytical solution for the flux into a semi-infinite slab with constant source.

Figure 4 Permeation history for a slab subject to effective-diffusivity limit trapping.

Figure 5 Permeation transient in a slab subject to strong-trapping. $\ldots \ldots \ldots 36$

Figure 6 Comparison of TMAP4 results with theory for concentration build-up by diffusion in a composite-layer material with a constant source on one side. 
Figure 7 TMAP4 calculations agreed well with theoretical values for both equal and unequal starting reactant concentrations.

Figure 8 There is virtually no difference between TMAP4 calculations and analytical results for the case of concentration transients in multiple chambers. .......................

Figure 9 Computed permeation transient which is essentially a duplicate of experimental data from an ion-implantation experiment. ...... 50

Figure 10 Comparison of calculated results (line) with experimental data $(+++)$ for thermal desorption test of beryllium after charging

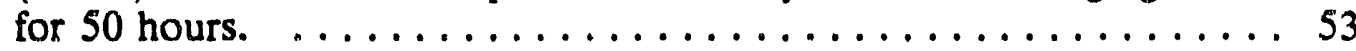

Figure 11 Comparison of TMAP4 calculational result with actual experimental data for the test cell release experiment. 55

\section{TABLES}

Table 1 Main Input Analysis $\ldots \ldots \ldots \ldots \ldots \ldots \ldots \ldots \ldots \ldots$

Table 2 Enclosure Input Analysis $\ldots \ldots \ldots \ldots \ldots \ldots \ldots \ldots \ldots$

Table 3 Thermal Segment Analysis $\ldots \ldots \ldots \ldots \ldots \ldots \ldots \ldots . \ldots 8$

Table 4 Diffusion Segment Analysis $\ldots \ldots \ldots \ldots \ldots \ldots \ldots \ldots$

Table 5 Control Input Analysis $\ldots \ldots \ldots \ldots \ldots \ldots \ldots \ldots \ldots \ldots \ldots$

Table 7 Coverage Analysis of TMAPP4 $\ldots \ldots \ldots \ldots \ldots \ldots \ldots \ldots$

Table 8 TMAPC4 Coverage Analysis $\ldots \ldots \ldots \ldots \ldots \ldots \ldots \ldots \ldots \ldots$

Table 9 Complexity Report by Subprogram for TMAPP4 $\ldots \ldots \ldots \ldots \ldots 20$

Table 10 Complexity Report by Subprogram for TMAPC4 $\ldots \ldots \ldots \ldots \ldots 21$

Table 11 Comparison of TMAP4 and Analytical Results for a

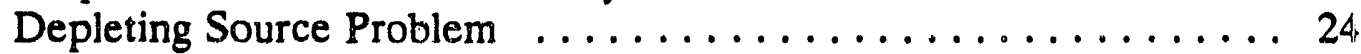


Table 12 Comparison of TMAP4 and Analytic Solution for

Diffusion Problem with Constant Source Boundary Condition:

Concentration History at $\mathrm{x}=0.2 \mathrm{~m} \ldots \ldots \ldots \ldots \ldots \ldots \ldots . \ldots \ldots$

Table 13 Comparison of TMAP4 with the Analytic Solution for

Semi-Infinite Slab with Constant Source:

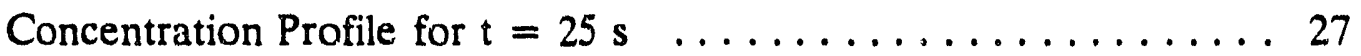

Table 14 Comparison of TMAP4 with the Analytic Solution for

Semi-Infinite Slab with Constant Source:

Flux Rate into the Surface . . . . . . . . . . . . . . . . 29

Table 15 Comparison of TMAP4 with the Analytic Solution for

Diffusion Problem with Partially Pre-loaded Slab at $\mathrm{x}=12, \mathrm{~m} \quad \ldots 31$

Table 16 Comparison of TMAP4 and Analytical Solution for

Concentration History at $\mathrm{x}=0$ in a Partially Preloaded

Semi-infinite Slab

Table 17 Comparison of TMAP4 and Analytical Solution for

Concentration History at $\mathrm{x}=10 \mathrm{~m}$ in a Partially Preloaded

Semi-infinite Slab

Table 18 Comparison of TMAP4 and Analytical Solution for

Concentration History (atom $\left./ \mathrm{m}^{3}\right)$ at $\mathrm{x}=8 \mu \mathrm{m}$ in a Composite

Material

Table 19 Comparison of TMAP4 and Analytical Solution for

Heat Sink/Source Problem with Internal Heat Generation . . . . . 40

Table 20 Comparison of TMAP4 and Analytical Results for

Simulation of a Thermal Transient by a Mass-Diffusion Transient . . 42

Table 21 Comparison of TMAP4 and Analytical Results for

Simple Chemical Reaction Problem with Equal Starting

Concentrations $\ldots \ldots \ldots \ldots \ldots \ldots \ldots \ldots \ldots \ldots \ldots, 4$,

Table 22 Comparison of TMAP4 and Analytical Results for

Simple Chemical Reaction Problem with Unequal

Starting Concentrations $\ldots \ldots \ldots \ldots \ldots \ldots \ldots \ldots . \ldots \ldots$

Table 23 Comparison of TMAP4 and Analytical Results for a

Multiple Enclosure Problem ................... 48 


\section{INTRODUCTION}

The Tritium Migration Analysis Program MOD1/CY04 (TMAP4) was developed by the Fusion Safety Program at the Idaho National Engineering Laboratory by EG\&G Idaho, Inc. as a tool for analyzing processes and systems that involve the interaction of tritium or other hydrogen isotopes with structures and enclosures that make v.p a fusion reactor system. However, it is much more versatile than that. The code accomplishes heat and mass transfer calculations in one-dimensional structures and associated zerodimensional enclosures. Specific processes encompassed are local heat generation and mass addition (by implantation, for example), diffusion, dissociation and recombination at surfaces, trapping and release from traps in structures, chemical reactions in enclosure volumes, and flows between enclosures and across enclosure-structure interfaces. A detailed description of the code is provided in the TMAP4 User's Manual. ${ }^{1}$ There are two parts to the code that are subject to verification and validation. One is the preprocessor that converts the user-written input deck into a form appropriate for analysis, including a compilable FORTRAN subprogram called TAPE7.FOR if equations are used in the input file. The other is the computational module that actually performs the calculations.

To be eligible for use in analyses supporting or serving as a basis for decision involving safety-related systems, structures, components, or services where failure could cause undue risks to employees or to the public health and safety, a computer code must be certified to meet Quality Level A. The process of achieving that certification within EG\&G Idaho is specified by Nuclear Engineering Standard Practice NE-SP-1 which $d$ lineates the requirements for preparing a Software Quality Assurance Plan. Those requirements are based on industry standards: ANSI/TEEE Standard 730-1984 for Software Quality Assurance Plans, ANSI/IEEE. Standard 830-1984 for Software Requirements Specifications, ANSI/IEEE Standard 1012-1986 for Software Verification 
and Validation Plans, and ANSI/LEEE Standard 828-1983 for Software Configuration Management Plans.

In sumnary, a Software Quality Assurance Plan (SQAP) is prepared that delineates requirements for the code itself, the verification and validation process, and configuration control. A number of tests are made on the code to assure that (1) the coding is correct and consistent with the theoretical model on which it is based, (2) the code performs in accordance with the stated characteristics in the code documentation, and (3) the results are consistent with physical observations or known solutions over the range of problems for which the certification is made. Success in reaching those objectives is determined by a Software Quality Assurance Board (SQAB). This report documents that process for TMAP4 operating on an IBM PS/2 Model 70 with DOS 5.0.

Once the code has been verified and validated, it is placed under Software Configuration Control (SCC) procedures which are under the direction of a Software Configuration Control Coordinator (SCCC). The SCCC maintains the permanent record copy and a backup copy of the code and all supporting documentation. Any changes or maintenance done on the code must conform to the requirements set forth in the SQAP and be approved by the SQAB.

The certification is done for a specific operating environment, in this case an IBM PS/2 Model 70 operating under DOS 5.0. Before certification may be granted to an changes or upgrades in the code or to the existing code in a different operating environment, the same process of verification and validation must be undertaken for the changed circumstances. Only when it has been determined that results in the new environment are equivalent to (or better if flaws are found in the original) the original code may the SQAB grant certification for that environment and/or the new configuration. 
In what follows we describe in detail the requirements and the work done to satisfy the requirements for verification and validation of TMAP4. The requirements are specified in the Software Quality Assurance Plan for TMAP4, Fusion Safety Program and Fuels and Materials Unit, June 24, 1992. The processes of verification and validation have uncovered several weaknesses in the code. These do not impact the accuracy of calculations, but do suggest improvements for future development iterations.

\section{VERIFICATION}

The following is extracted from the SQAP as a definition of the tasks required for verification of TMAP4.

The verification of the preprocessor stage is required to ensure that all applicable diagnostic error detection and processing of all user entered data performs intended and designed. This verification assessment will use one or more input models to exercise all available input options in TMAP4. It will involve extensive and systematic assessments of error diagnostic detection of each input parameter within the input deck which is composed of several input blocks. The process will impose systematic errors at each applicable parameter position within an input strearn. four types of formats will be used for each possible input parameter: 1) a hollerith format, 2) an integer number format, 3) a real (fixed decimal) number format, and 4) an exponential number format. One format will always be the correct format. for each imposed error type, the resulting error message will be assessed to assure a proper "error processed" execution termination, as well as the appropriateness of the diagnostic response(s). For each selected input deck, the results of all four error format types will be documented along with any generated diagnostic error messages related to the imposed error. The documentation will also include the original error-free input deck(s) to allow error re-construction audits.

To limit the scope of this assessment, several items will be excluded: 1) the "Title Input" block since it only performs title input processing and has no effect on the final quality level of this code, 2) repeated input parameters, such as those encountered in multiple diffusion and thermal segments and multiple table definition inputs since they utilize the same set of error checking statements [Note: Repeated segments and tables will be checked by multiple duplication of already verified segments/tables - up to the allowed limits (see Section 3.4)], and 3) the "Equation Input" block since its error assessments are performed by an external FORTRAN compiler. 
In what follows we describe in detail the requirements and the work done to satisfy the requirements for verification and validation of TMAP4. The requirements are specified in the Software Quality Assurance Plan for TMAP4, Fusion Safety Program and Fuels and Materials Unit, June 24, 1992. The processes of verification and validation have uncovered several weaknesses in the code. These do not impact the accuracy of calculations, but do suggest improvements for future development iterations.

\section{VERIFICATION}

The following is extracted from the SQAP as a definition of the tasks required for verification of TMAP4.

The verification of the preprocessor stage is required to ensure that all applicable diagnostic error detection and processing of all user entered data performs as intended and designed. This verification assessment will use one or more input models to exercise all available input options in TMAP4. It will involve extensive and systematic assessments of error diagnostic detection of each input parameter within the input deck which is composed of several input blocks. The process will impose systematic errors at each applicable parameter position within an input stream. four types of formats will be used for each possible input parameter: 1) a hollerith format, 2) an integer number format, 3) a real (fixed decimal) number format, and 4) an exponential number format. One format will always be the correct format. for each imposed error type, the resulting error message will be assessed to assure a proper "error processed" execution termination, as well as the appropriateness of the diagnostic response(s). For each selected input deck, the results of all four error format types will be documented along with any generated diagnostic error messages related to the imposed error. The documentation will also include the original error-free input deck(s) to allow error re-construction audits.

To limit the scope of this assessment, several items will be excluded: 1) the "Title Input" block since it only performs title input processing and has no effect on the final quality level of this code, 2) repeated input parameters, such as those encountered in multiple diffusion and thermal segments and multiple table definition inputs since they utilize the same set of error checking statements [Note: Repeated segments and tables will be checked by multiple duplication of already verified segments/tables - up to the allowed limits (see Section 3.4)], and 3) the "Equation Input" block since its error assessments are performed by an external FORTRAN compiler. 
The main transient execution stage of the code will be verified in two parts.

The first part will utilize several complex models (limited to the ones developed for the preprocessor validation) to show the functionality of all available options and to allow general assessments of the validity of the predicted results (i.e., no flow through "nonflow" boundary conditions, depletion of source concentrations, development of expected diffusion gradients, etc.). These input decks will be documented and described along with a qualitative description of the expected and obtained numerically predicted results.

The second part of the main transient execution stage verification will use preprocessor verified input models to provide a general assessment of the TMAP4 program structure. This process will utilize the Coverage Analysis Tools available at the INEL by the Scientific Computing Unit (\#4C20, POC: Earl Marwil or designated alternate) to identify major logic paths, to assess the amount of executable code utilized, to check subroutine utilization efficiencies, etc. The objective of this second stage is to identify (and eliminate if necessary) any unused program coding and to assure that all applicable program structure has been utilized. The documentation of this stage of the verification will again require a description and listing of the chosen input decks and must provide a summary and description of the Coverage Analysis Tools results.

Verification was accomplished in two parts. The first addressed the preprocessor module and was mainly concerned with error detection. The second exercised both the preprocessor and computational module to ensure proper operation and to apply the coverage analysis techniques available. Validation was an exercise to establish the agreement between TMAP4 results and manual analytical solutions for selected problems. We now discuss these in detail.

\section{Preprocessor}

Verification of the preprocessor module of TMAP4 is required to ensure that all applicable diagnostic eiror detection anci processing of all user-entered data perform as intended and designed. Systematic assessments of the TMAP4 input options have been performed through a process which imposes specitied errors at each applicable parameter locácion within a given input parameter stream. For each imposed error type, 
The main transient execution stage of the code will be verified in two parts. The first part will utilize several complex models (limited to the ones developed for the preprocessor validation) to show the functionality o: all available options and tc allow general assessments of the validity of the predicted results (i.e., no flow through "nonflow" boundary conditions, depletion of source concentrations, development of expected diffusion gradients, etc.). These input decks will be documented and described along with a qualitative description of the expected and obtained numerically predicted results.

The second part of the main transient execution stage verification will use preprocessor verified input models to provide a general assessment of the TMAP4 program st ucture. This process will utilize the Coverage $A_{\text {Analysis }}$ Tools available at the INEL by the Scientific Computing Unit (\#4C20, POC: Earl Marwil or designated alternate) to identify major logic paths, to assess the amount of executable code utilized, to check subroutine utilization efficiencies, etc. The objective of this second stage is to identify (and eliminate if necessary) any unused program coding and to assure that all applicable program structure has been utilized. The documentation of this stage of the verification will again require a description and listing of the chosen input decks and must provide a summary and description of the Coverage. Analysis Tools results.

Verification was accomplished in three parts. The first addressed the preprocessor module and was mainly concerned with error detection. The second exercised both the preprocessor and computational module to ensure proper operation and to apply the coverage analysis techniques available. The third was an exercise to establish the agreement between TMAP4 results and manual analytical solutions for selected problems. We now discuss these in detail.

\section{Preprocessor}

Verification of the preprocessor module of TMAP4 is required to ensure that all applicable diagnostic error detection and processing of all user-entered data perform as intended and designed. Systematic assessments of the TMAP4 input options have been performed through a process which imposes specified errors at each applicable parameter location within a given input parameter stream. For each imposed error type, 
the resulting error message was assessed to assure proper error processing, appropriateness of the error response, and execution termination.

Verification of the preprocessor included an error assessment of seven of the rine input blocks. Those included were:
1. Main Input
1. Enclosure Input
2. Thermal Segment Input
3. Diffusion Segment Input
6. Table Input
5. Controi ingat
7. Plot Input

The Title Input block was ignorew since it has no computational effect on the execution of the code. The Equation Input block was also omitted since error assessments are handled externally by the FORTRAN compiler. Assessment of the code's irlput limits as stated in Section 3.4 of the SQAP is implicit in this analysis.

Two approaches were utilized in imposing specified errors at each parameter location based upon whether the input parameter required single parameter inputs (may or may not be repeating) or multiple data values. A single parameter input is illustrated as follows:

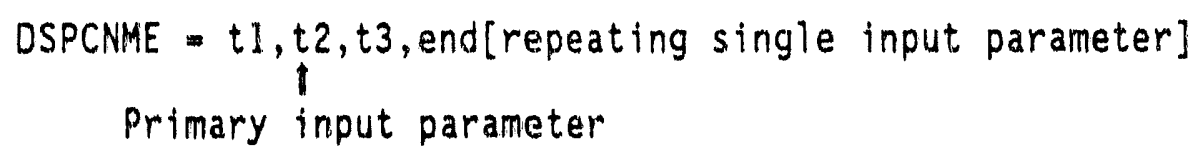

In this case, the primary input parameter is varied between a real, and integer, an hollerith, or an exponential format. Only one parameter is varied as the resulting error message is common to all of them.

A multiple data input parameter differs from the single input as shown in the following example: 


\section{Secondary Input Parameter \\ $\downarrow$ \\ HTRBCL $=$ CONVEC, const, 1.5, ENCL, 1 , end \\ Primary Input Parameter}

The primary input may take the form of a repeating data value, a constant qualifier with a single data value (as shown above), or an equation or table identifier with a single data value, while the secondary input is typically a single parameter of a specified format. For this type of input stream, only the primary input parameter was formally assessect. The secondary input was varied from its specified format to assure proper error detection and termination, but formal presentation of this data is not presented. The results for the input blocks listed previously are presented in Tables 1 through 6 . Results for Table Input are not presented in tabular form because this section was only checked for consistency with the stated format in the manual. This input block was found to behave as stated in the TMAP4 User's Manual.

Table 1 Main InPUt Analysis

\begin{tabular}{|c|c|c|c|c|}
\hline Main & Real & Integer. & Hollerith & Exponential \\
\hline DSPCNME & D & $\square$ & 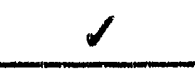 & $\square$ \\
\hline ESPCNME & $\square$ & $\square$ & $\checkmark$ & $\square$ \\
\hline SEGNDS & 1 & $\checkmark$ & ${ }^{1}$ & $2^{1}$ \\
\hline NBRENCL & $\mathbf{B}^{2}$ & $\checkmark$ & $\Delta^{2}$ & $8^{2}$ \\
\hline LINKSEGS & $\mathrm{B}^{3}$ & $d$ & $0^{3}$ & $\mathbf{8}^{3}$ \\
\hline
\end{tabular}

Key: Results are as expected

Error message received; see \# below for error message.

口Alphabetic format is indicated in manual - no message returned.

\section{Error Messages:}

$1^{\prime \prime}$...nbr. of seg. nodes at word -2- is not an integer value."

2 "...integer value expected after + nbrencl.$+ "$

$3^{\prime \prime}$...integer segment nbr. expected." 


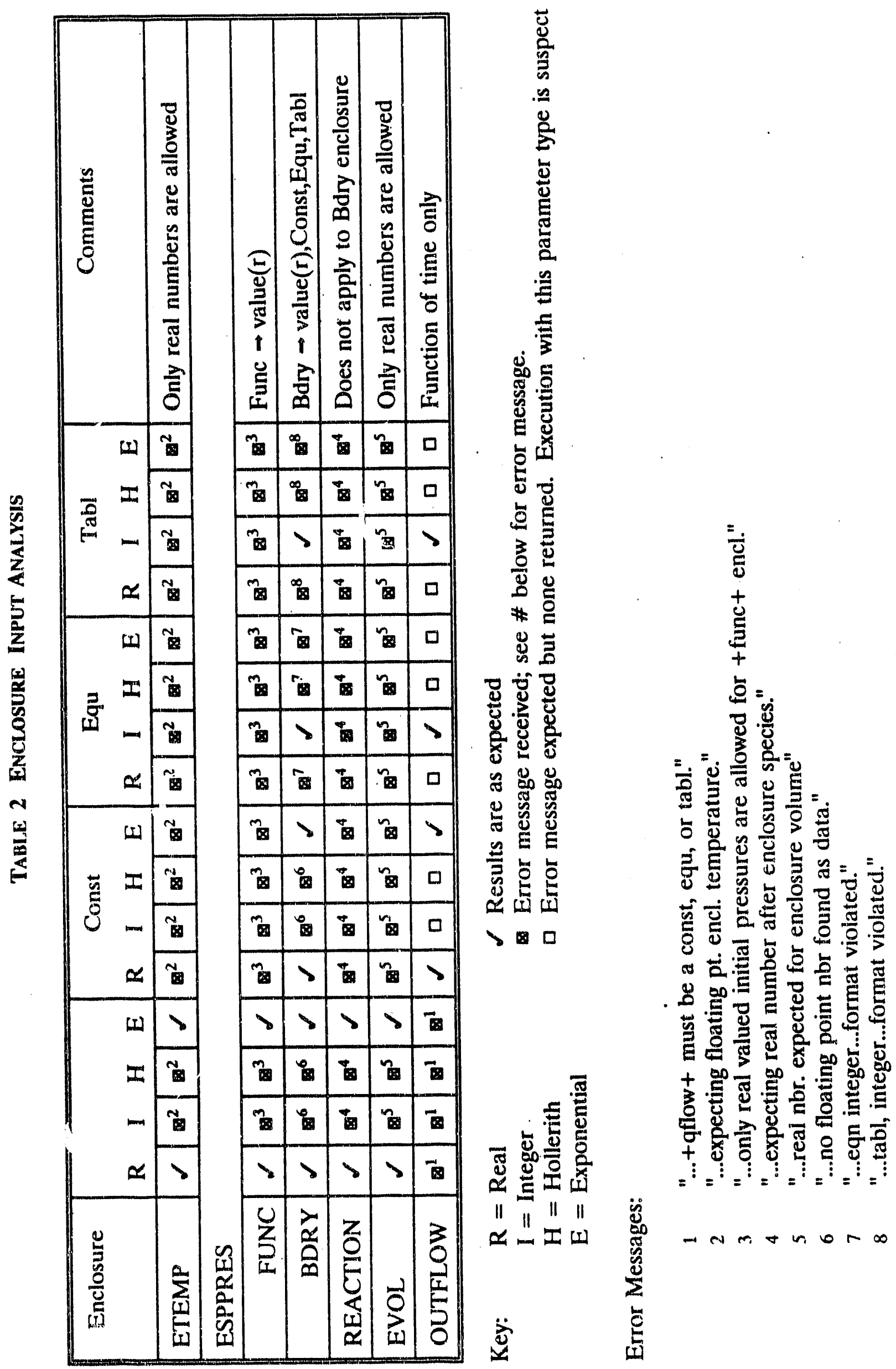




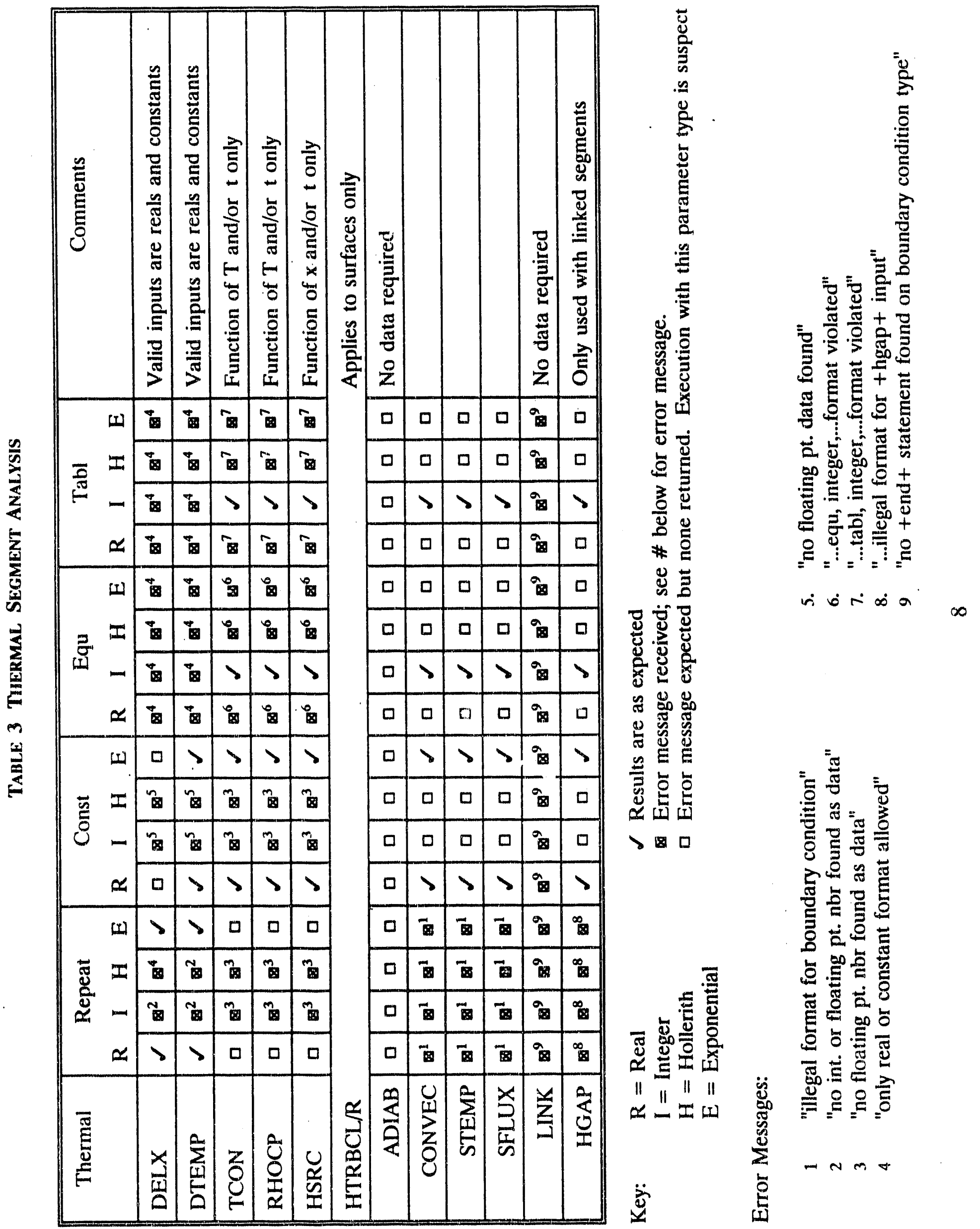




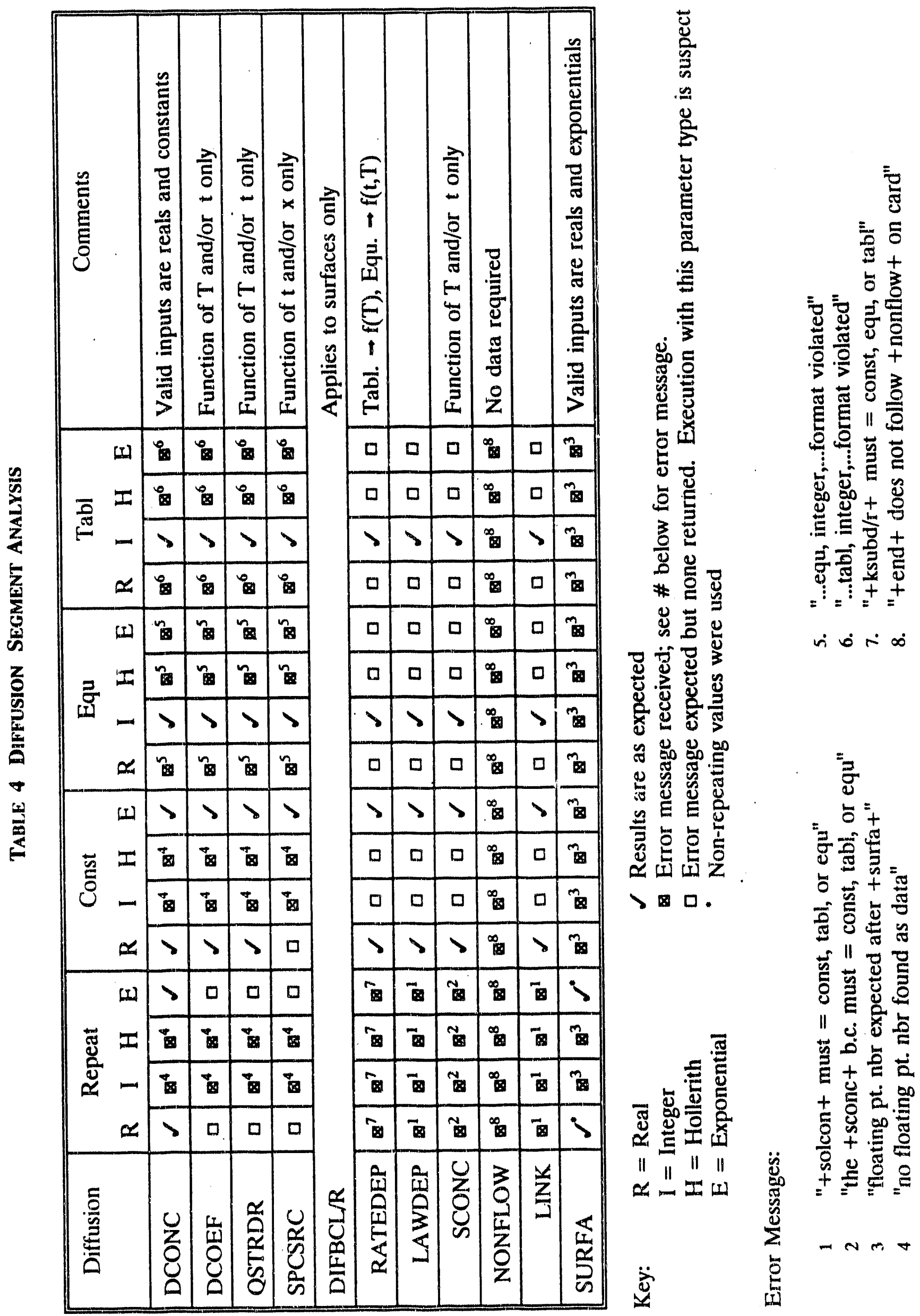


TABLE 5 CONTROL INPUT ANALYSIS

\begin{tabular}{|c|c|c|c|c|}
\hline Main & Real & Integer & Hollerith & Exponential \\
\hline TIME & $\checkmark$ & $\square$ & $\square$ & $\checkmark$ \\
\hline TSTEP & $\checkmark$ & $\square$ & $\square$ & $\checkmark$ \\
\hline TIMEND & $\checkmark$ & $\square$ & $\square$ & $\checkmark$ \\
\hline NPRINT & $\square$ & $\checkmark$ & $\square$ & $\square$ \\
\hline ITERMX & $\square$ & $\checkmark$ & $\square$ & $\square$ \\
\hline DELCMX & $\checkmark$ & $\square$ & $\square$ & $\checkmark$ \\
\hline
\end{tabular}

Key: $\quad \checkmark \quad$ Results are as expected

- Error message expected but none returned. Execution with this parameter type is therefore suspect.

TAble 6 Plot InPUT ANAlysis

\begin{tabular}{|c|c|c|c|c|}
\hline Main & Real & Integer & Hollerith & Exponential \\
\hline NPLOT & $\square$ & $\checkmark$ & $\square$ & $\square$ \\
\hline PLOTSEG & $\square$ & $\checkmark$ & $\mathbf{a}^{1}$ & $\square$ \\
\hline PLOTENCL & $\square$ & $\checkmark$ & $\mathbf{8}^{2}$ & $\square$ \\
\hline DNAME & (2) & (1) & $(1)$ & (2) \\
\hline ENAME & (1) & () & 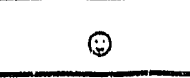 & () \\
\hline DPLOT & $\checkmark$ & $\mathcal{L}$ & $\checkmark$ & $\downarrow$ \\
\hline EPLOT & $\checkmark$ & $\checkmark$ & $\checkmark$ & $\checkmark$ \\
\hline
\end{tabular}

Key: $\quad \checkmark \quad$ Results are as expected

Error message received; see \# below for error message.

- Error message expected but none returned. Execution with this parameter type is therefore suspect.

(1) Alphabetic input specified. Execution will proceed if D/ESPCNME and D/EPLOT are consistent.

Error Messages:

1 "...expecting integer for diffusion segment."

2 "...expecting integer for enclosure number." 
Additional input restrictions observed were that a "reaction array out-of-bounds" error will result when greater than 20 reactions are attempted. Also, it was confirmed that the "end" statement must immediately follow a delimiter such as a comma or a carriage return. It may not appear on a line by itself unless it begins in column 1.

There were numerous instances in the tables preceding where error messages were expected for a given data type, but none were returned. Execution of the code with these erroneous inputs needs to be performed to assess the effect these parameters impose. Additionally, error checking routines will need to be included or modified to flag these invalid data parameters. Both issues will be addressed in the next code revision and release. Correct input data type results in correct results, and we have elected not tc make changes suggested by $\square$ in the tables at this time.

\section{Computational Module Analysis}

The following analysis was provided by the Scientific Computing Unit of EG\&G Idaho, Inc. It represents an independent assessment of the quality of the code from the perspective of specialists in scientific computing who were not previously acquainted with it. Some specific comments they present will be replied to in following sections.

The TMAP4 program consists of a preprocessor program, TMAPP4, and a computational processor, TMAPC4. Thirty-seven sample problems were supplied along with the source code. All files were uploaded from a Macintosh PC to the INEL Cray X/MP-216 for assessment and analysis. Each program was analyzed using the CRAFT (Cross Reference Analysis of FORTRAN) tool, FORWARN, the FORTRAN 77 analyzer, and PC-Metric. These tools provide static analysis, coverage analysis, and complexity analysis. 


\section{Functions}

TMAPP 4 contains one function, nextcrd, with an alternate entry point, incivi: One function, wrtcrd, is not called by any routine in TMAPP4. ${ }^{\text {a }}$

There were no alternate entry points in TMAPC4 and no unreferenced functions.

\section{Common Block Irregularities}

The common blocks used in both codes are generally consistent. A checkpoint and restart capability of the code uses equivalencing as a technique to write and read data from and to certain common blocks. This technique contributes to making the code less readable and more difficult to trace the use of variables used in those common blocks. The equivalence of a local array name (of length 1) to an array in common (of length greater than 1) generates a warning message. The arrays should be of the same size to avoid potentially introducing defects during code maintenance..

In the program TMAPP4, the following definition irregularities were observed: The array cardc in common block card 2 is undefined and used. The variable iblank in common block cmno is defined but unused. The variable $n r a$ in common block cmnOa is defined but unused. There are a number of instances where a common block is declared in a module but none of its elements are used or modified. These could be removed from the code to reduce clutter and improve readability.

Since data from TMAPP4 is written to disk and read by program TMAPC4, some of the following require additional analysis to determine whether they contribute to program clutter or are related to the equivalencyg techniques cited above. The arrays idseg and ithrm in common block connect are apparently defined but unused. The array iqdot in common block instr is also apparently defined but unused. Variables and arrays icouple, ncyc, itermx, nprint, nloop, itera, nplotd, nplote,named, namee, and iplot in common block integ are apparently defined but unused. Arrays and variables asurf, srcse,

\footnotetext{
The alternate entry point is accessed while reading and processing the data statements in the input deck and is particularly useful when an input statement extends beyond one line. The subroutine wrtcrd, was included as a means of conveniently displaying the values read and processed by the preprocessor. It is a utility for code improvement and maintenance and should not be accessed during user operation of the code. It could be removed with no consequence to code performance, but is retained for future convenience.
} 
vole, qdot, cetrpi, and dennum in common block names are apparently defined but unused.

In TMAPC4 there are a number of entities in common with potential definition irregularities (undefined and unused, defined and unused, or used but undefined). Further analysis would be necessary to determine whether these are defined when reading the binary input data file generated by . TMAPP4. There are a number of instances where a common block is declared in a module but none of its elements are used or modified. These could be removed from the code to reduce clutter and improve readability.

In some blocks, an implicit equivalence of two variable names sharing the same memory location occurs where the names differ by one character and one variable name is used in only one module. These include: icbe and ipbe in block instr, ncbe and npbe in instr, and itera and iterct in block integ.

\section{Interface Irregularities}

In both TMAPP4 and TMAPC4 there are local variables which are apparently defined but unused. A further inspection is required to determine if these are actually used or whether they contribute to program clutter.

In addition, in TMAPP4 routines dimchk, instr2, instr3, and relnbr have an argument which is a fixed length character variable. The calling routines use a character constant. This situation frequently results in a length conflict between the character constant and the character variable. (Most compilers simply truncate or pad the constant to the appropriate length.) An easy change to avoid this problem is to declare the character variable to have variable length in the called routines.

\section{FORTRAN Extensions}

FORTRAN 77 requires that entity names be no longer than 6 characters. Some entity names in TMAPP4 and TMAPC4 are 7 characters long. This is an extension to the language which is recognized by most compilers. FORTRAN 90 allows up to 31 characters in a name. With the need for more meaningful naming conventions, long entity names should not be shortened to conform to the standard.

The TMAPP4 and TMAPC4 programs are written using lower case characters. This is an extension to the FORTRAN 77 standard.

There is extensive use of the REAL*8 and INTEGER* 4 type declarations. The use of the integer constant in this context is nonstandard. 
In several places a character array element is replaced by a substring of the same element concatenated with another character variable. This is nonstandard due to the potential overlap in a character assignment statement.

\section{Coverage Analysis}

Thirty-seven sample problems were supplied. A coverage analysis shows that these problems yield an $80 \%$ segment coverage of TMAPP4 and an $87 \%$ segment coverage of TMAPC4. Tables 7 and 8 show the percent coverage for each routine.

\section{Complexity Analysis}

The complexity measures for both codes indicate very few block IF's and many unconditional GO TO statements. The code was probably developed after the FORTRAN 77 standard was established, but using a style more suited to FORTRAN 66. In addition, the codes have a good ratio of nonblank comments to source code.

Some key metrics are McCabe's cyclomatic complexity, the number of lines of source code, a density of McCabe's cyclomatic complexity per 100 lines of source code, and the number of unconditional GO TO statements. Generally, the routines with the highest complexity are those most likely to have defects. A software maintenance program should focus on those routines with the highest measures.

Details of the complexity analysis appear in Tables 9 and 10 for TMAPP4 and TMAPC4, respectively.

\section{Developer Comments}

A careful review of the "defined but not used" and "used but not defined" diagnostics revealed that with three exceptions, all of the variables listed in the above report are appropriately defined and used. Prima facie eivdence for that being the case is that the code executes and gives good results. That would not happen if variables were used without being defined. They are defined in TMAPP4 by "data" statements or read in from the "tmapinp" file and passed to TMAPC4 through the "tape1" file where they are restored to the common blocks for use. Exceptions are the variable names 
cardc, iblank, and nra which are defined in TMAPP4 but appear in no other context. Hence, they appear to be excess baggage that could be removed. 


\section{TABLE 7 COVERAge ANALYSIS OF TMAPP4}

0

BANNER

CARDCV

CARDRD

CONTROL

DIFF

DIFFBC

DIMCHK

ENCLOSE

ERRCHK

ERRCVRY

FLOW

GENFLOW

GENREAC

HSOURCE

INCIW

INSTR2

INSTR3

LOAD

LOX

MAINP

PLOT

REACTIN

READE

READRS

READT

REFORM

RELNBR

SPECDEP

SPECPFS

STORE

THERM

THERMBC

TITLE

TMAPP4

TRAPIN

WRTBLKS

WRTCRD
0.20

0.40

0.60

0.80

1.00

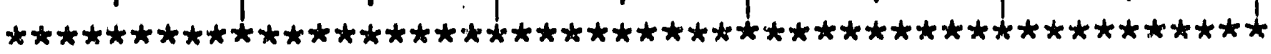

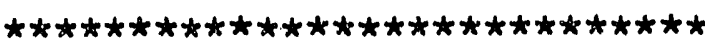

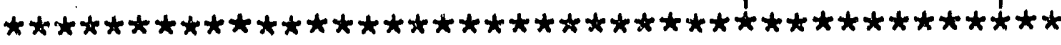

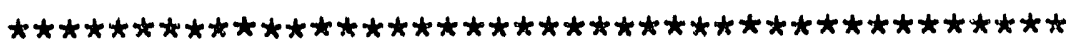

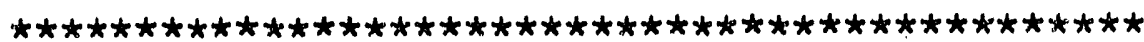

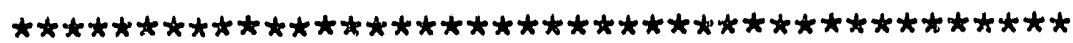

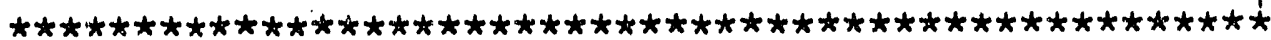

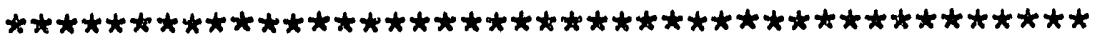

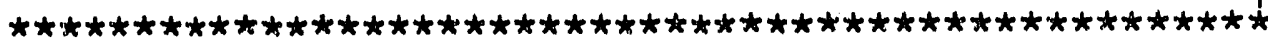

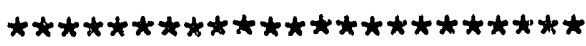

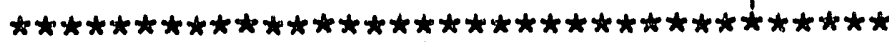

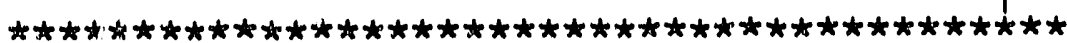

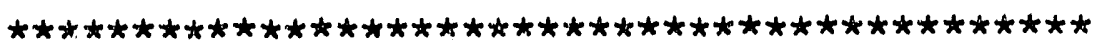

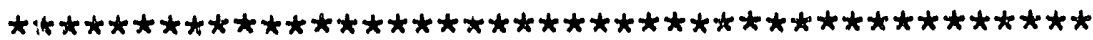

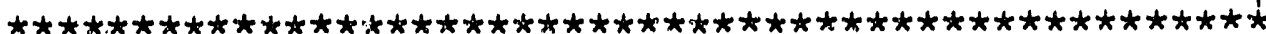

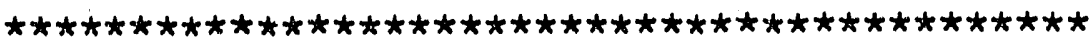

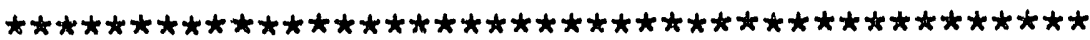

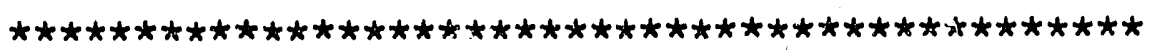

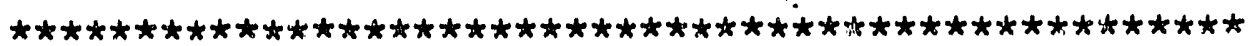

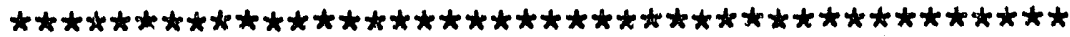

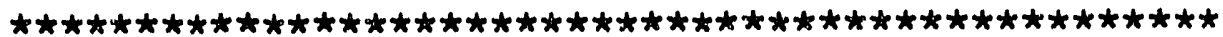

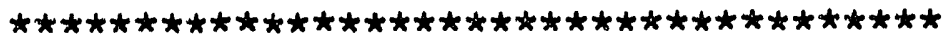

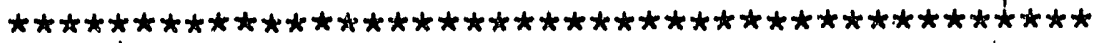

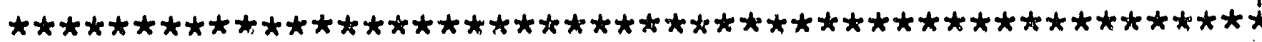

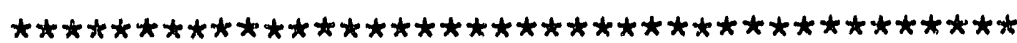

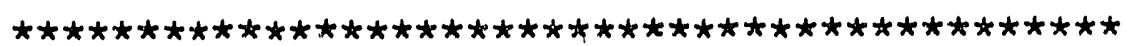

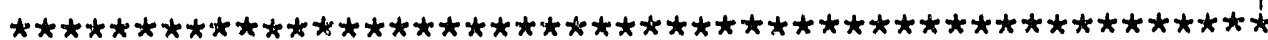

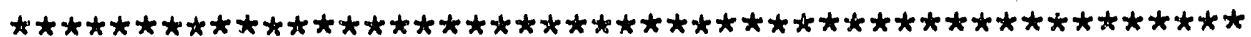

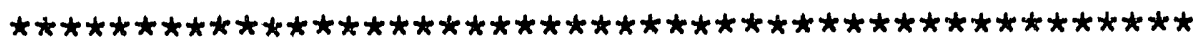

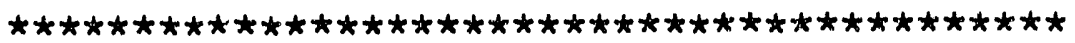

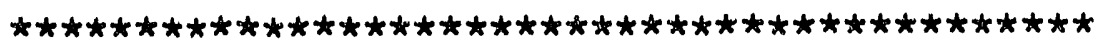

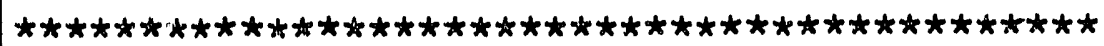

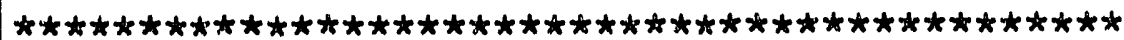

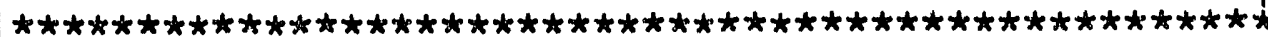

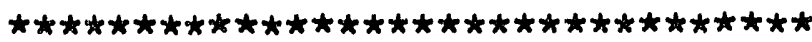

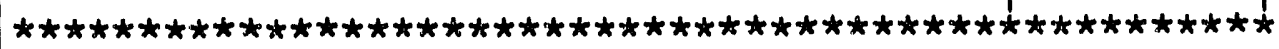

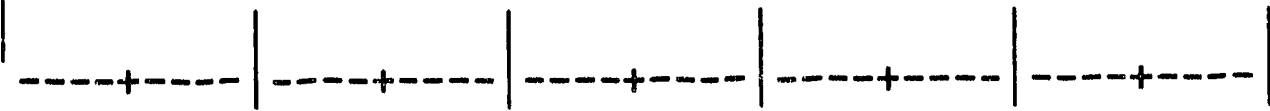




\begin{tabular}{|c|c|c|c|c|c|}
\hline & coverage $=0$ & WRTCRD & & . & \\
\hline 0.40 & $<=$ coverage $<0.60$ & CARDCV & ERRCVRY & & \\
\hline 0.60 & $<=$ coverage $<0.80$ & FLOW & REACTIN & TRAPIN & \\
\hline 0.80 & $<=$ coverage $<0.85$ & $\begin{array}{l}\text { CARDRD } \\
\text { MAINP }\end{array}$ & $\begin{array}{l}\text { CONTROL } \\
\text { READT }\end{array}$ & $\begin{array}{l}\text { DIFFBC } \\
\text { STORE }\end{array}$ & GENFLOW \\
\hline 0.85 & $<=$ coverage $<0.90$ & $\begin{array}{l}\text { ENCLOSE } \\
\text { INSTR3 } \\
\text { THERMBC }\end{array}$ & $\begin{array}{l}\text { GENREAC } \\
\text { READE } \\
\text { TITLE }\end{array}$ & $\begin{array}{l}\text { HSOURCE } \\
\text { REFORM }\end{array}$ & $\begin{array}{l}\text { INSTR2 } \\
\text { THERM }\end{array}$ \\
\hline 0.90 & $<$ coverage $<0.95$ & DIFF & LOAD & SPECPFS & \\
\hline 0.95 & $<=$ coverage $<1.00$ & PLOT & SPECDEP & & \\
\hline & coverage $=1.00$ & $\begin{array}{l}\text { BANNER } \\
\text { LOX } \\
\text { TMAPP4 }\end{array}$ & $\begin{array}{l}\text { DIMCHK } \\
\text { READRS } \\
\text { WRTBLKS }\end{array}$ & $\begin{array}{l}\text { ERRCHK } \\
\text { RELNBR }\end{array}$ & INCIW \\
\hline
\end{tabular}




\section{TABLE 8 TMAPC4 COVERAGE ANALYSIS}

D1DEG1

DIFFUSE

ENCLOSE

INCWRD

ITER

OUTPUT

PLOT

READBLK

SIMQ

TABLKP

THERM

TMAPC

TRIDAG

UPDSHRC

UPDMDCO

UPDPRES

UPDQDOT

UPDQSTR

UPDRHCP

UPDSSRC

UPDTCON

UPDTRPR

UPDTRPT

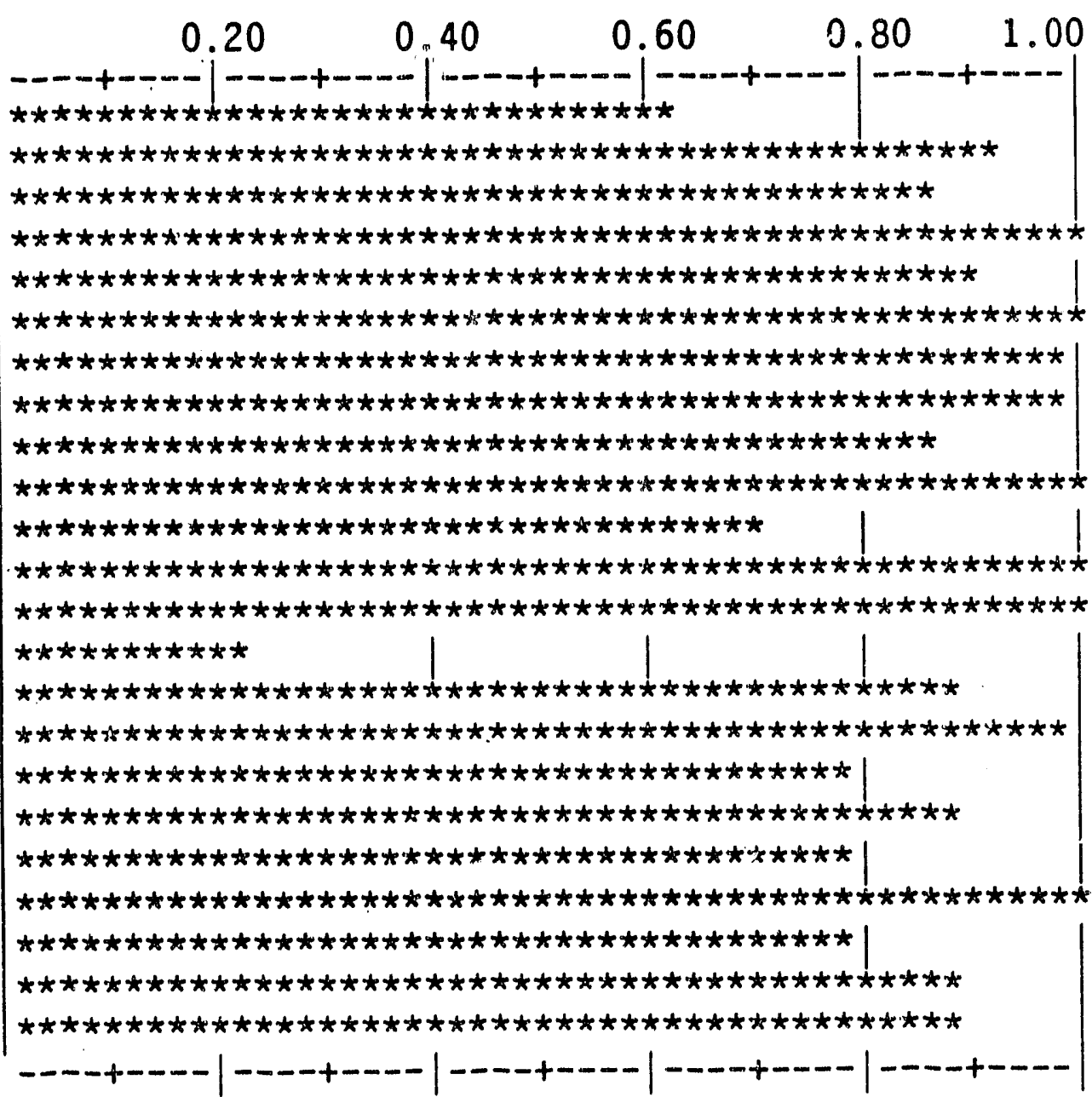


$0.20<$ coverage $<0.40$ UPDHSRC

$0.60<=$ coverage $<0.80$ DIDEGI THETCON THERM UPDQDOT UPCIRHCP

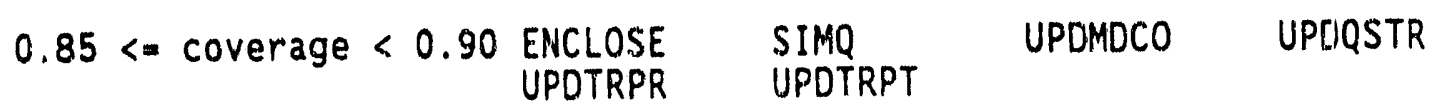

$0.90<$ = coverage $<0.95$ DIFFUSE ITER

0.95 \& coverage < 1.00 PLOT READBLK

$\begin{array}{lllll}\text { coverage }=1.00 & \begin{array}{l}\text { INCWRD } \\ \text { TRIDAG }\end{array} & \begin{array}{l}\text { OUTPUT } \\ \text { UPDPRES }\end{array} & \begin{array}{l}\text { TABLKP } \\ \text { UPDSSRC }\end{array} & \text { TMAPC }\end{array}$

Cumulative coverage $=0.087$ 
8.

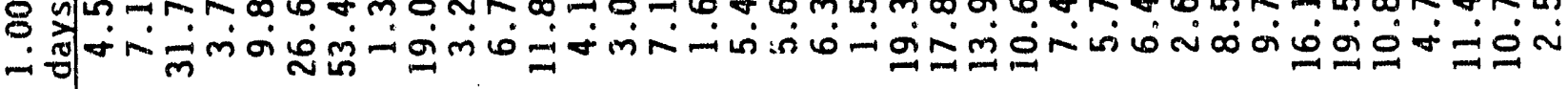

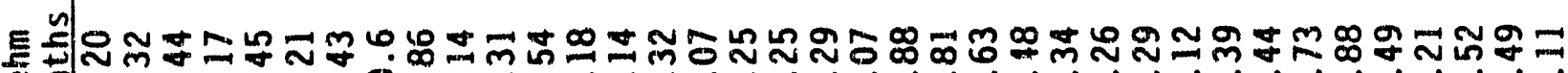

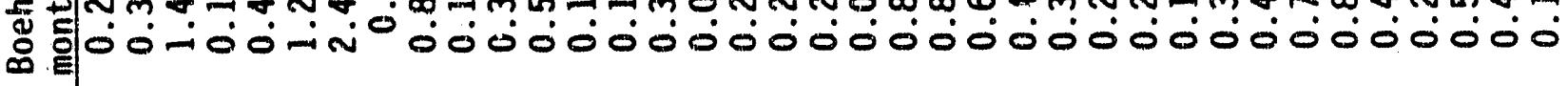
n n - 0 -

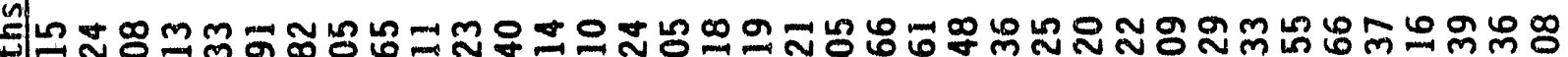

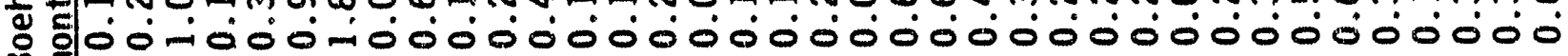

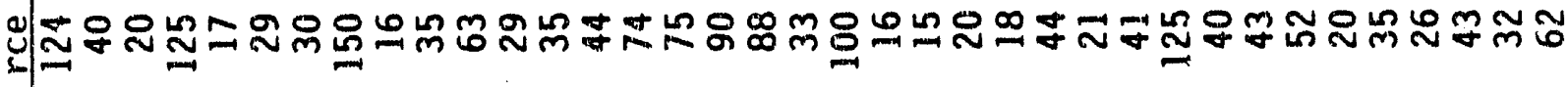

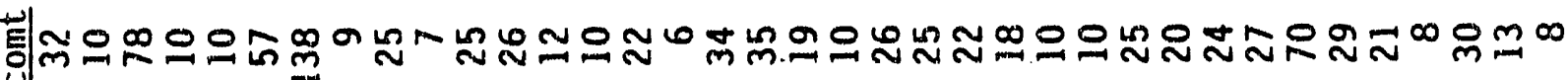
$\underline{y}$

8

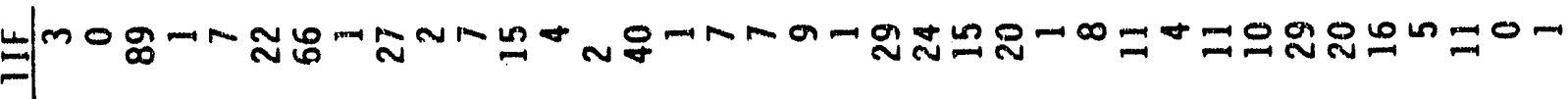

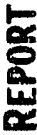

$=0000010000000000000000000000000000000$

잉ㅇㅇㅇㅁㅛ

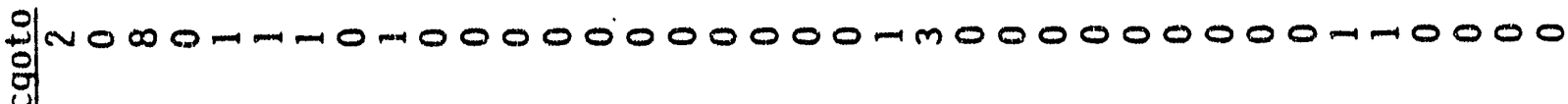

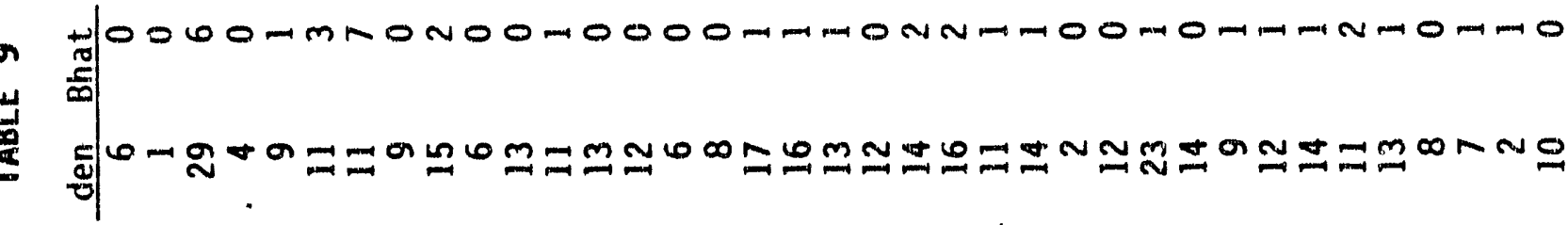

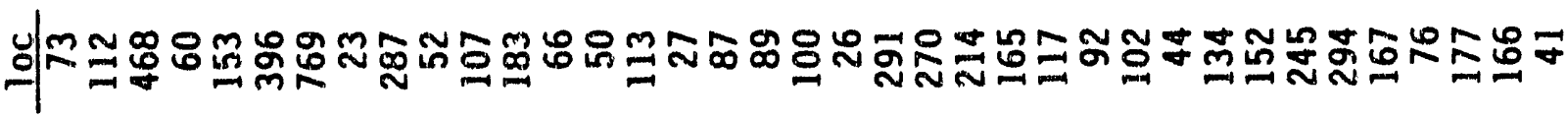

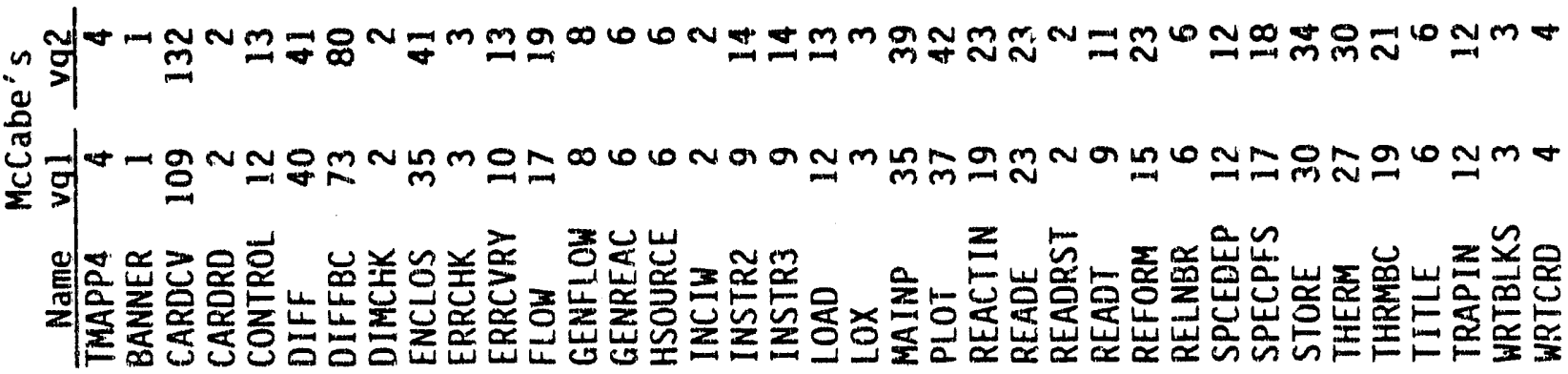


8 ㄴำm nom no

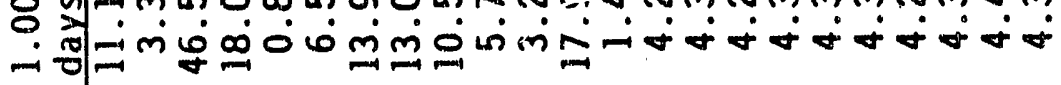

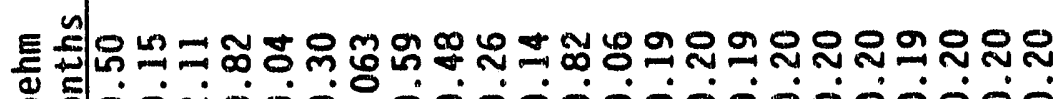

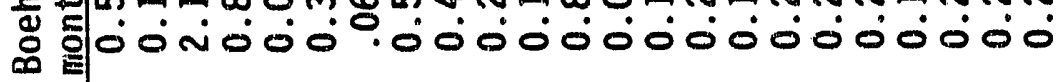
10.

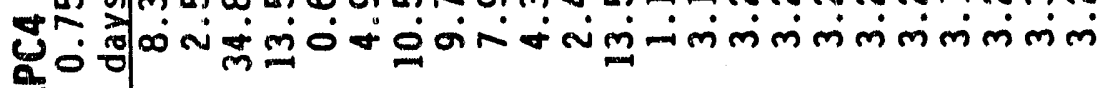

틀 今.

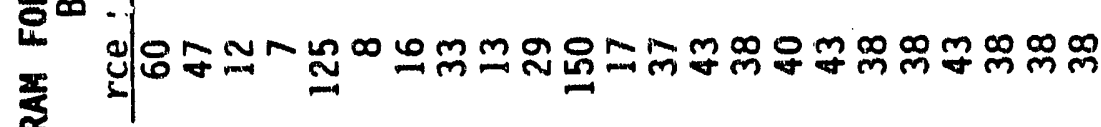

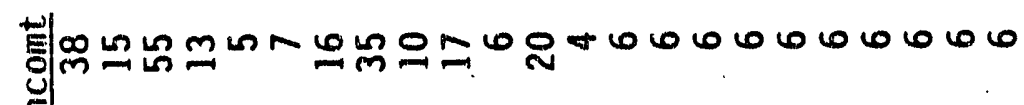

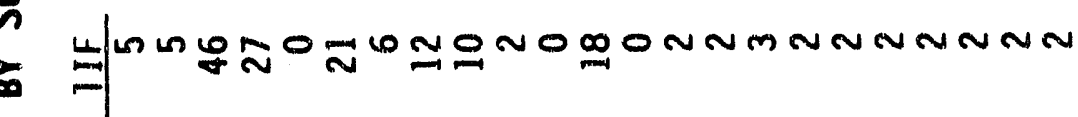
능 400000000000000000000000

E 윰 홍 웡

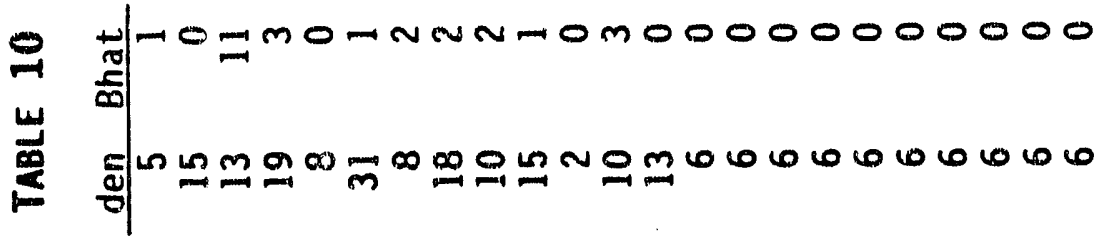

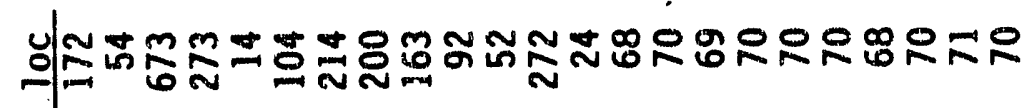

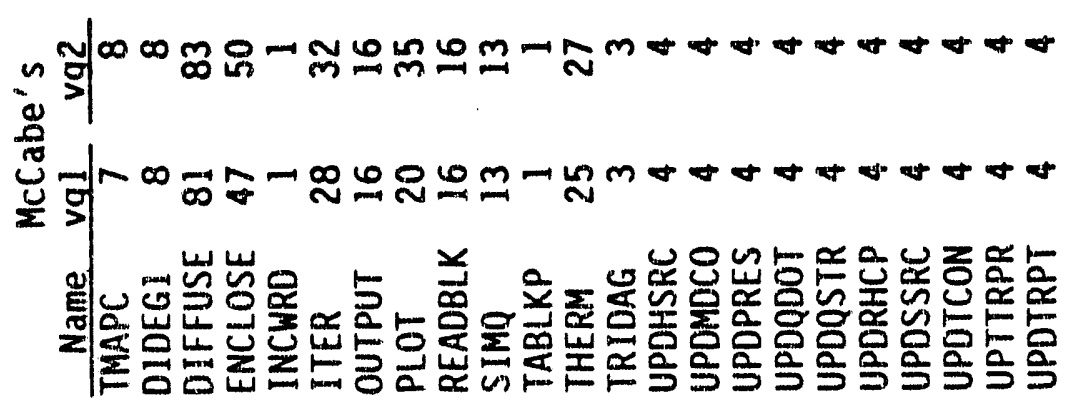

4 


\section{VALIDATION}

The validation assessment of the TMAP4 code is presented in this section. It was done in two parts as outlined in the SQAP. First, comparisons of selected numerical models to analytical solutions were generated. Then comparisons with three sets of experimental results were made. The validation assessment includes descriptions of the input deck along with a copy of the deck on the supplementary disk. Output files are also provided on the supplementary disk, and a comparison is provided here of the calculated results with both theoretical and experimental results.

\section{Comparison with Analytic Solutions}

The specific problems to be examined under this phase of validation are listed in the TMAP4 SQAP. The SQAP identified eight such problems for comparison. Filenames for the input files used in these problems are given in parentheses after the problem subheading.

\section{1a) Depleting Source Problem ${ }^{2}$ (val-1a.inp)}

This model consists of an enclosure containing a finite concentration of atoms which are allowed to diffuse into a $\mathrm{SiC}$ layer over time. No solubility or trapping effects are included. The fractional release from the outside of the shell in a depleting source model in a slab geometry is given by:

$$
F R=1-\sum_{n=1}^{\infty} \frac{2 L \sec \alpha_{n} \exp \left(-\alpha_{n}^{2} \frac{D T}{l^{2}}\right)}{L(L+1)+\alpha_{n}^{2}}
$$

where

$$
L=\frac{l A}{V \phi}
$$




$$
\phi=\frac{\text { source concentration }}{\text { layer concentration }}
$$

where the layer concentration is that at the interface with the source ( $\phi$ is constant in time),

$$
\begin{aligned}
& A=\text { surface area } \\
& V=\text { source volume } \\
& l=\text { layer thickness }
\end{aligned}
$$

and the $\alpha_{n}$ are the roots of

$$
\alpha_{n}=\frac{L}{\tan \alpha_{n}}
$$

The results of TMAP4's calculated release are shown in Table 11 along with the analytical solution. Column 4 of the table presents the variation of TMAP4's calculated results on the IBM PS $2 / 70$ compared with the analytical solution expressed as:

$$
\text { Variation }=\frac{\text { TMAP4 } \cdot \text { Value }- \text { Analytical Value }}{\text { Analytical value }}
$$

Except for the early times (i.e., 0 - 10 seconds), agreement between TMAP4 and the analytical solution are generally within $1 \%$ with TMAP4 overpredicting the fractional release. At later times, TMAP4 slightly underpredicts. The analytical solution was obtained by discarding series terms with a contribution of less than 1.0E-10 using Lotus $123^{\mathrm{m}}$ release 3.0 on and IBM PS 2/70. Table 11 presents the initial 45 seconds of a 140 second analysis. Beyond 45 seconds, the variation between the two results remains less than $1 \%$. Figure 1 shows the comparison in graphic format. 
TABle 11 COMParison OF TMAP4 AND ANALYTICAL RESUlts fOR A Depleting Source Problem

\begin{tabular}{|c|c|c|c|}
\hline Time (s) & TMAP4 & Theory & Variation \\
\hline 0 & 0 & 0 & 0 \\
\hline 1 & 0.000644 & $6.0 \mathrm{E}-07$ & 1081.268 \\
\hline 2 & 0.002831 & 0.000262 & 9.806189 \\
\hline 3 & 0.007223 & 0.002399 & 2.010316 \\
\hline 4 & 0.014077 & 0.007873 & 0.7881 \\
\hline 5 & 0.023316 & 0.016781 & 0.389429 \\
\hline 6 & 0.034657 & 0.028554 & 0.213738 \\
\hline 7 & 0.047736 & 0.042499 & 0.12323 \\
\hline 8 & 0.058001 & 0.06218 & 0.072051 \\
\hline 9 & 0.077649 & 0.074568 & 0.041318 \\
\hline 10 & 0.093853 & 0.091823 & 0.022112 \\
\hline 11 & 0.110548 & 0.109482 & 0.009737 \\
\hline 12 & 0.127545 & 0.127337 & 0.001637 \\
\hline 13 & 0.144693 & 0.145233 & -0.00372 \\
\hline 14 & 0.161873 & 0.163059 & -0.00727 \\
\hline 15 & 0.17899 & 0.180733 & -0.00964 \\
\hline 16 & 0.195987 & 0.198197 & -0.01115 \\
\hline 17 & 0.212795 & 0.21541 & -0.01214 \\
\hline 18 & 0.22939 & 0.232343 & -0.01271 \\
\hline 19 & 0.245738 & 0.248977 & -0.01301 \\
\hline 20 & 0.261816 & 0.265301 & -0.01314 \\
\hline 21 & 0.27761 & 0.281307 & -0.01314 \\
\hline 22 & 0.293121 & 0.296991 & -0.01303 \\
\hline 23 & 0.30833 & 0.312353 & -0.01288 \\
\hline 24 & 0.323243 & 0.327395 & -0.01268 \\
\hline 25 & 0.337859 & 0.342119 & -0.01245 \\
\hline 26 & 0.352174 & 0.356529 & -0.01222 \\
\hline 27 & 0.366192 & 0.37063 & -0.01197 \\
\hline 28 & 0.379914 & 0.384426 & -0.01173 \\
\hline 29 & 0.393354 & $0.397923^{\prime}$ & -0.01148 \\
\hline 30 & 0.406503 & 0.411126 & -0.01125 \\
\hline 31 & 0.419375 & 0.424042 & -0.01101 \\
\hline 32 & 0.43197 & 0.436677 & -0.01078 \\
\hline 33 & 0.444295 & 0.449035 & -0.01056 \\
\hline 34 & 0.456355 & 0.461122 & -0.01034 \\
\hline 35 & 0.46815 & 0.472945 & -0.01014 \\
\hline 36 & 0.4797 & 0.484509 & -0.00993 \\
\hline 37 & 0.490991 & 0.49582 & -0.00974 \\
\hline 38 & 0.502044 & 0.506883 & -0.00955 \\
\hline 39 & 0.512857 & 0.517703 & -0.00936 \\
\hline 40 & 0.52343 & 0.528286 & -0.00919 \\
\hline 41 & 0.533777 & 0.538637 & -0.00902 \\
\hline 42 & 0.543904 & 0.54876 & -0.00885 \\
\hline 43 & 0.55381 & 0.558662 & -0.00869 \\
\hline 44 & 0.563495 & 0.568346 & -0.00854 \\
\hline 45 & 0.572973 & 0.577818 & -0.00838 \\
\hline
\end{tabular}




\section{Depleting Source Problem}

SQAP Reference 4.2.1.a

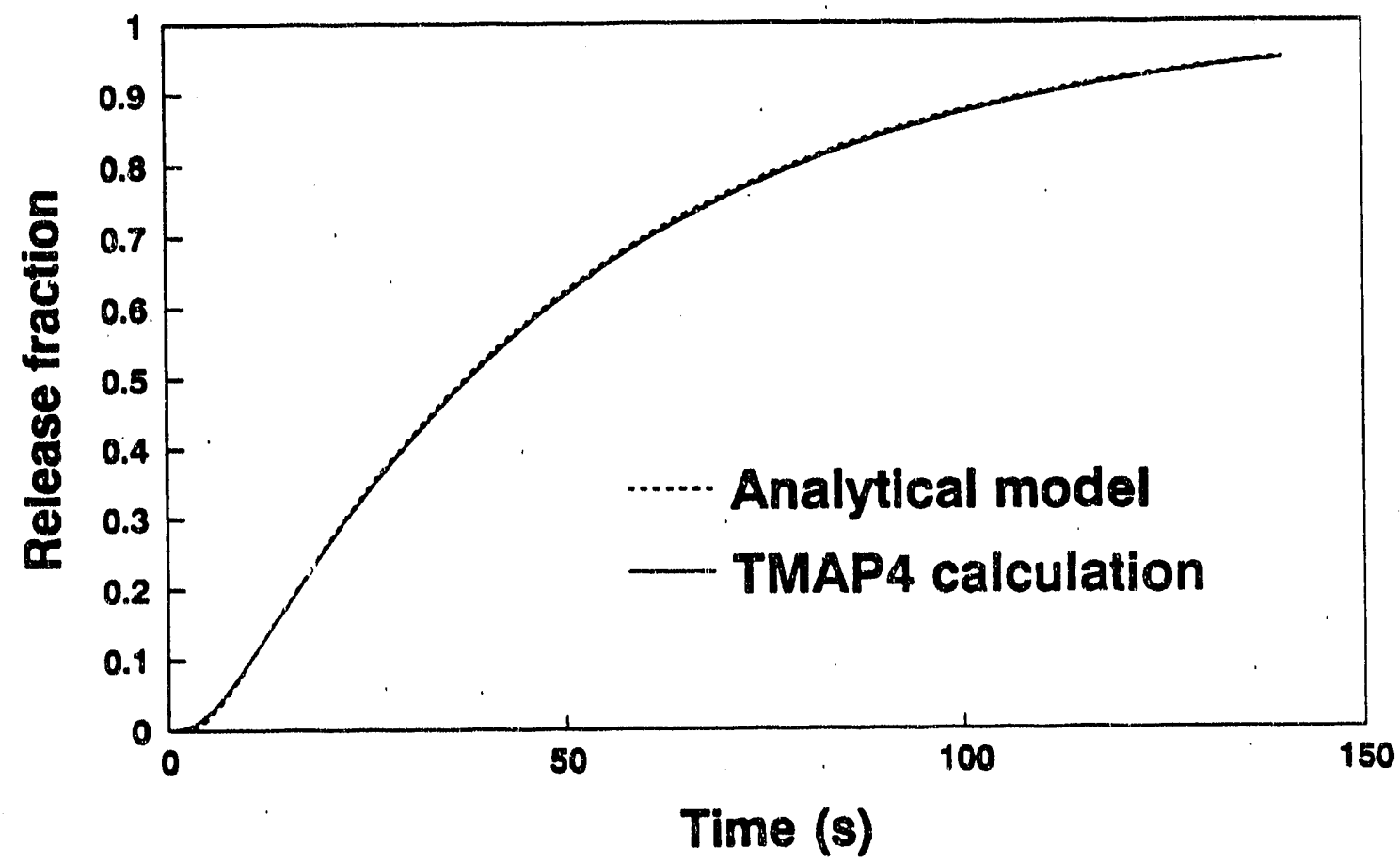

Figure 1. TMAP4 gives excellent results in comparison with the analytical solution for the depleting source problem.

1b) Diffusion Problem with Constant Source Boundary Condition ${ }^{2}$ (val-1b.inp)

Diffusion of tritium through a semi-infinite $\mathrm{SiC}$ layer is modelled with a constant source located on one boundary. No solubility or trapping is included. The concentration as a function of time and position is given by

$$
C=C_{0} \operatorname{erfc}\left(\frac{x}{2 \sqrt{D t}}\right)
$$

Comparison of the TMAP4 results on an IBM PS $2 / 70$ and the analytical solution developed using Lotus $123^{\mathrm{m}}$ Version 3.0, taking 26 terms in the series solution for erfc $(x)$, is given in Table 12 as a function of time at $\mathrm{x}=0.2 \mathrm{~mm}$. For simplicity, both the diffusion :oefficient and the initial concentration were set to unity. Agreement between 
the code predictions and Equation (2) is very good with TMAP4 overpredicting the concentration by less than $1 \%$ for times greater than one second.

Table 12 Comparison of TMAP4 and ANalytic Solution for Diffusion Problem With Constant Source Boundary Condition: CONCENTRATION History AT $\mathrm{X}=0.2 \mathrm{M}$

\begin{tabular}{|c|c|c|c|}
\hline Time & TMAP4 & Theory & Variation \\
\hline 0 & 0 & 0 & 0 \\
\hline 1 & 0.85969 & 0.887537 & -0.03138 \\
\hline 2 & 0.92516 & 0.920344 & 0.005232 \\
\hline 3 & 0.94379 & 0.934925 & 0.009482 \\
\hline 4 & 0.95315 & 0.943628 & 0.010091 \\
\hline 5 & 0.95901 & 0.949571 & 0.00994 \\
\hline 6 & 0.9631 .2 & 0.95396 & 0.009602 \\
\hline 7 & 0.96622 & 0.957372 & 0.009242 \\
\hline 8 & 0.96866 & 0.960122 & 0.008892 \\
\hline 9 & 0.97067 & 0.962401 & 0.008592 \\
\hline 10 & 0.97235 & 0.964329 & 0.008317 \\
\hline 11 & 0.97379 & 0.965988 & 0.008076 \\
\hline 12 & 0.97506 & 0.967436 & 0.007881 \\
\hline 13 & 0.97618 & 0.968712 & 0.007709 \\
\hline 14 & 0.97717 & 0.96985 & 0.007548 \\
\hline 15 & 0.97808 & 0.970872 & 0.007424 \\
\hline 16 & 0.97889 & 0.971796 & $(1.007299$ \\
\hline 17 & 0.97964 & 0.972638 & 0.007199 \\
\hline 18 & 0.98032 & 0.973409 & 0.0071 \\
\hline 19 & 0.98095 & 0.974118 & 0.007014 \\
\hline 20 & 0.98153 & 0.974773 & 0.006932 \\
\hline 21 & 0.98207 & 0.975381 & 0.006858 \\
\hline 22 & 0.98257 & 0.975947 & 0.006787 \\
\hline 23 & 0.98303 & 0.976475 & 0.006713 \\
\hline 24 & 0.98345 & 0.97697 & 0.006632 \\
\hline 25 & 0.98385 & 0.977435 & 0.006563 \\
\hline 26 & · $\quad 0.98422$ & 0.977874 & 0.00649 \\
\hline 27 & 0.98457 & 0.978287 & 0.006422 \\
\hline 28 & 0.98489 & 0.978678 & 0.006347 \\
\hline 29 & 0.9852 & 0.979049 & 0.006283 \\
\hline 30 & 0.98548 & 0.979401 & 0.006207 \\
\hline
\end{tabular}


As a second check, the concentration as a function of position at a give time, $\mathrm{t}=25 \mathrm{~s}$, from TMAP4 was compared with Equation (2). Results are given in Table 13. Here the variation is seen to be smaller for small distances, $\mathbf{x}$, from the surface but it increases as $\mathrm{x}$ increases. This is shown graphically in Figure 2.

TABle 13 COMParison of TMAP4 With the ANALYTIC Solution for SEMI-INFINITE SLAB WITH CONSTANT SOURCE:

Concentration Profile for $\mathrm{T}=25 \mathrm{~S}$

\begin{tabular}{llll}
\hline $\mathbf{x}(\mathrm{m})$ & TMAP4 & Theory & Variation \\
0 & 1 & 1 & 0 \\
0.05 & 0.99462 & 0.994358 & 0.000263 \\
0.15 & 0.98385 & 0.983076 & 0.000788 \\
0.25 & 0.97309 & 0.971796 & 0.001331 \\
0.35 & 0.96234 & 0.960523 & 0.001892 \\
0.45 & 0.95159 & 0.949257 & 0.002458 \\
0.55 & 0.94086 & 0.938002 & 0.003047 \\
0.65 & 0.93014 & 0.926759 & 0.003649 \\
0.75 & 0.91944 & 0.91553 & 0.004271 \\
0.85 & 0.90876 & 0.904318 & 0.004912 \\
0.95 & 0.8981 & 0.893126 & 0.00557 \\
1.05 & 0.88747 & 0.881954 & 0.006254 \\
1.15 & 0.87686 & 0.870806 & 0.006952 \\
1.25 & 0.86629 & 0.859684 & 0.007684 \\
1.35 & 0.85574 & 0.848589 & 0.008427 \\
1.45 & 0.84523 & 0.837524 & 0.0092 \\
1.55 & 0.83476 & 0.826492 & 0.010004 \\
1.65 & 0.82433 & 0.815493 & 0.010836 \\
1.75 & 0.81394 & 0.804531 & 0.011695 \\
1.85 & 0.8036 & 0.793607 & 0.012592 \\
1.95 & 0.7933 & 0.782723 & 0.013512 \\
& & & \\
\hline
\end{tabular}




\section{Semi-infinite Constant Source Model SQAP Reference 4.2.1.b}

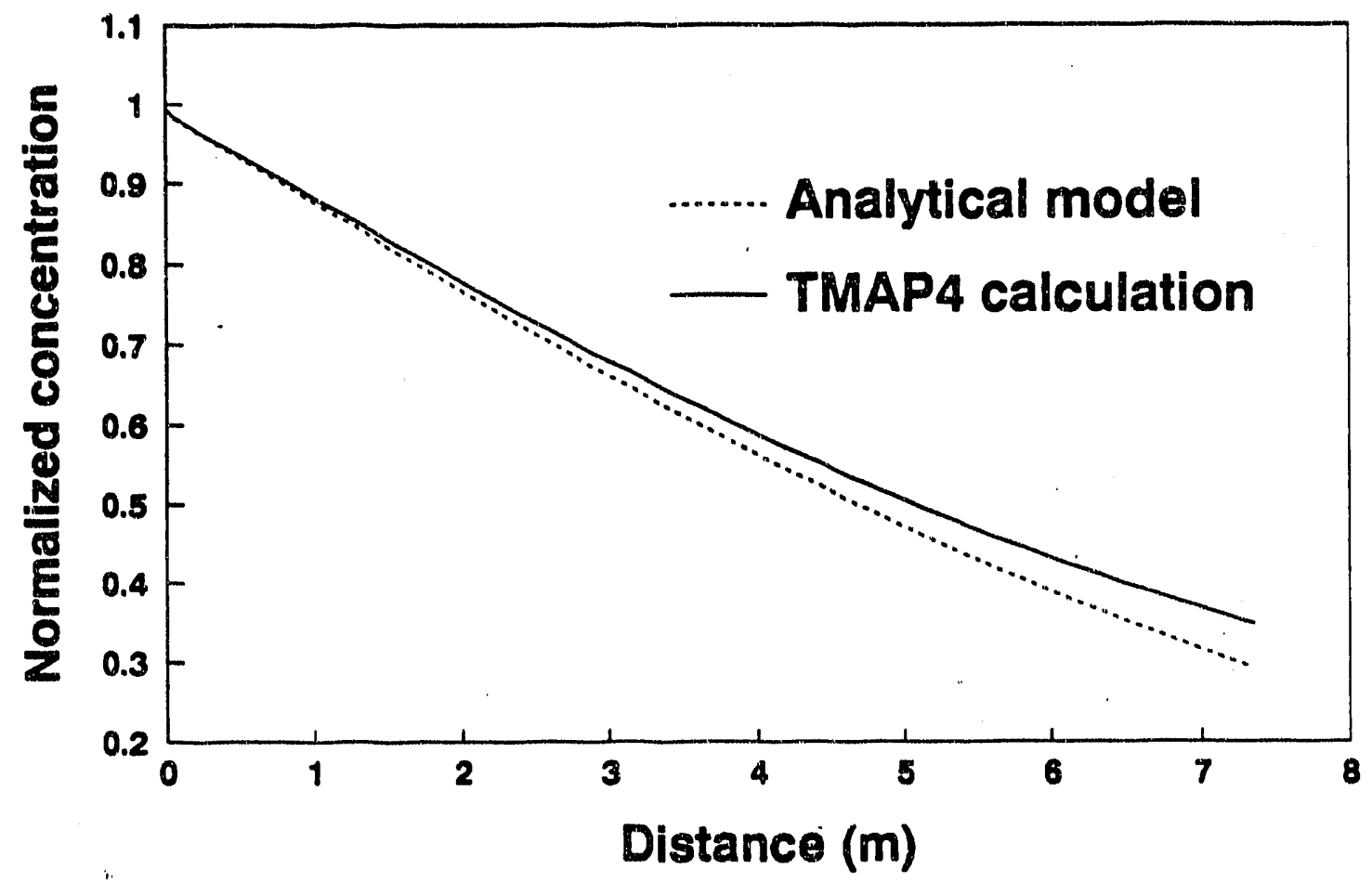

Figure 2. Concentration profile at $t=25 \mathrm{~s}$ for semi-infinite slab with constant source.

Finally, the diffusive flux, was compared with the analytic solution where the flux is proportional to the derivative of the concentration with respect to $\mathrm{x}$ and is given by

$$
J=C_{0} \sqrt{\frac{D}{\pi t}} \exp \left(\frac{x}{2 \sqrt{D t}}\right)
$$

The flux as given by Equation (3) is compared with values calculated by TMAP4 in Table 14. The diffusivity, $D$, and the initial concentration, $C_{o}$, were both taken as unity, and the distance, $x$, was taken as zero in this comparison. TMAP4 initially overpredicts because of the finite time step ( 1 second in this case). That may be reduced by making the time step smaller. Results are shown graphically in Figure 3. 
TABle 14 Comparison of TMAP4 With THE ANAlytic Solution For SEMI-INFINITE SLAB WITH Constant SourCE:

FLUX RATE INTO THE SURfaCE

\begin{tabular}{|c|c|c|c|}
\hline Time (s) & TMAP4 & Theory & Variance \\
\hline 0 & 1 & 1 & 0 \\
\hline 1 & 0.99875 & 0.56419 & 0.770238 \\
\hline 2 & 0.50062 & 0.398942 & 0.254868 \\
\hline 3 & 0.37514 & 0.325735 & 0.151672 \\
\hline 4 & 0.31254 & 0.282095 & 0.107925 \\
\hline 5 & 0.27339 & 0.252313 & 0.083534 \\
\hline 6 & 0.24594 & 0.230329 & 0.067775 \\
\hline 7 & 0.22528 & 0.213244 & 0.056444 \\
\hline 8 & 0.20896 & 0.199471 & 0.04757 \\
\hline 9 & 0.19561 & 0.188063 & 0.040129 \\
\hline 10 & 0.18438 & 0.178412 & 0.033448 \\
\hline 11 & 0.17473 & 0.17011 & 0.027162 \\
\hline 12 & 0.16631 & 0.162868 & 0.02113 \\
\hline 13 & 0.15886 & 0.156478 & 0.015222 \\
\hline 14 & 0.15219 & 0.150786 & 0.009311 \\
\hline 15 & 0.14618 & 0.145673 & 0.00348 \\
\hline 16 & 0.14073 & 0.141047 & -0.00225 \\
\hline 17 & 0.13575 & 0.136836 & -0.00794 \\
\hline 18 & 0.13119 & 0.132981 & -0.01347 \\
\hline 19 & 0.12699 & 0.129434 & -0.01888 \\
\hline 20 & 0.12313 & 0.126157 & -0.02399 \\
\hline 21 & 0.11955 & 0.123116 & -0.02897 \\
\hline 22 & 0.11624 & 0.120286 & -0.03363 \\
\hline 23 & 0.11317 & 0.117642 & -0.03801 \\
\hline 24 & 0.11031 & 0.115165 & -0.04215 \\
\hline 25 & 0.10766 & 0.112838 & -0.04589 \\
\hline 26 & 0.10518 & 0.110647 & -0.04941 \\
\hline 27 & 0.10287 & 0.108578 & -0.05257 \\
\hline 28 & 0.10071 & 0.106622 & -0.05545 \\
\hline 29 & 0.098693 & 0.104767 & -0.05798 \\
\hline 30 & 0.096801 & 0.103006 & -0.06024 \\
\hline 31 & 0.095027 & 0.101331 & -0.06222 \\
\hline 32 & 0.09336 & 0.099736 & -0.06392 \\
\hline 33 & 0.091792 & 0.098213 & -0.06538 \\
\hline 34 & 0.090316 & 0.096758 & -0.06658 \\
\hline 35 & 0.088924 & 0.095365 & -0.06754 \\
\hline 36 & 0.08761 & 0.094032 & -0.06829 \\
\hline 37 & 0.086368 & 0.092752 & -0.06883 \\
\hline 38 & 0.085191 & 0.091524 & -0.06919 \\
\hline 39 & 0.084076 & 0.090343 & -0.06937 \\
\hline 40 & 0.083017 & 0.089206 & -0.06938 \\
\hline
\end{tabular}




\section{Semi-infinite Constant Source Model}

SQAP Reference 4.2.1.b

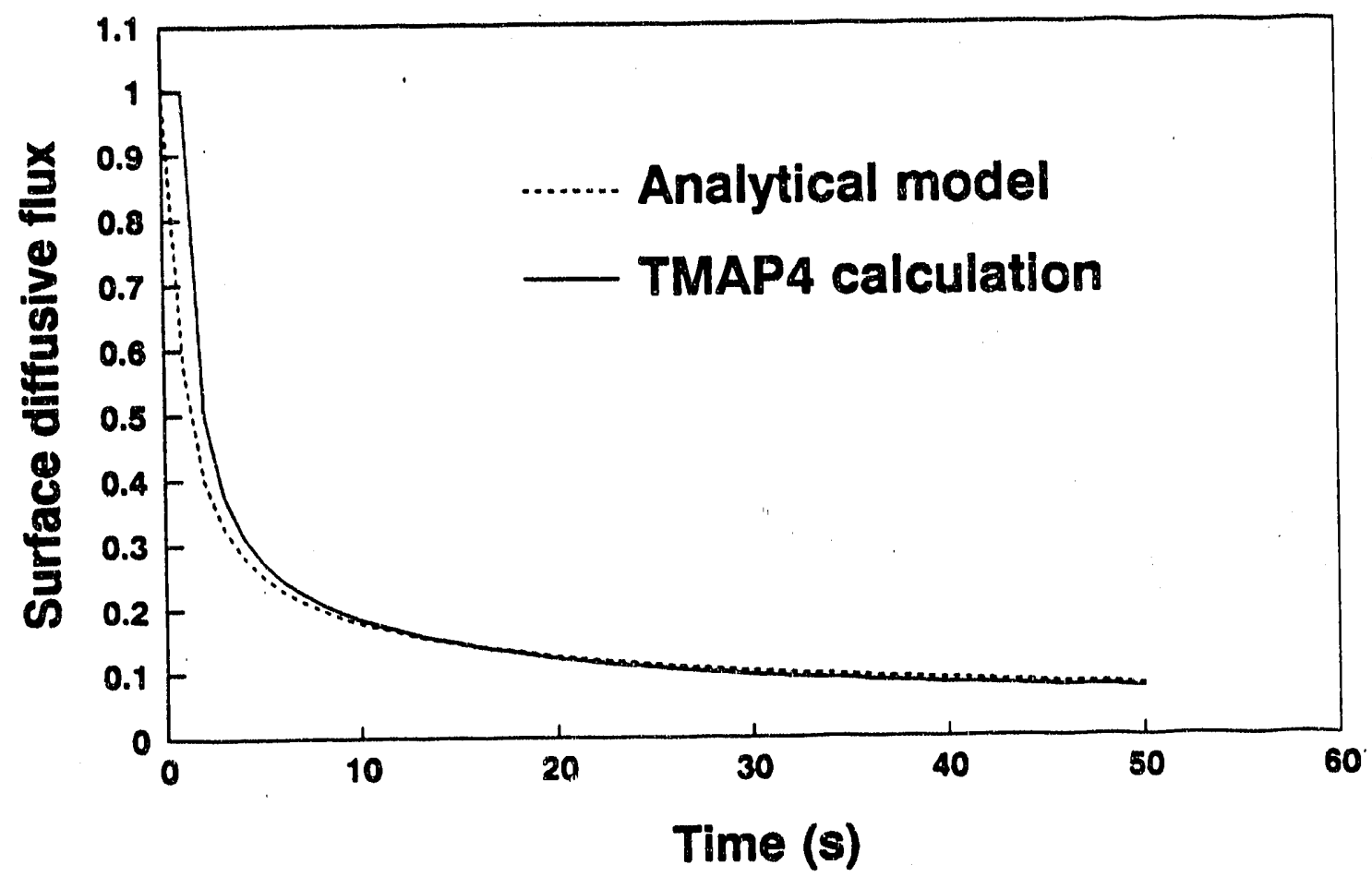

Figure 3. Comparison of TMAP4 calculation with the analytical solution for the flux into a semi-infinite slab with constant source.

1c) Diffusion Problem with Partially Preloaded Slab ${ }^{2}$ (val-1c.inp)

Diffusion of tritium through a semi-infinite SiC layer is modelled with an initial loading of 1 atom $/ \mathrm{m}^{3}$ in the first $10 \mathrm{~m}$ of a $2275-\mathrm{m}$ slab. Solubility is unity and no trapping is included. The analytical solution is given by

$$
C=\frac{C_{0}}{2}\left[\operatorname{eff}\left(\frac{h-x}{2} \sqrt{D t}\right)+\operatorname{erf}\left(\frac{h+x}{2 \sqrt{D t}}\right)\right]
$$

where $h$ is the thickness of the pre-loaded portion of the layer. The results for the concentration are shown in Table 15 as a function of time at $\mathrm{x}=12 \mathrm{~m}$. Note that this is 
obtained by taking the average of mobile species concentration values at $x=11.5 \mathrm{~m}$ (node 12) and $x=12.5 \mathrm{~m}$ (node 13 ) from the problem *.out file.

TABle 15 Comparison of TMAP4 WITH the ANALYTIC SOlUtion for Diffusion Problem with Partially Pre-loaded Slab at $x=12 \mathrm{M}$

\begin{tabular}{cllc}
\hline Time $(\mathrm{s})$ & TMAP4 & Theory & Variance \\
0 & 0.000000 & 0.000000 & 0.0000 \\
5 & 0.261690 & 0.263545 & -0.0070 \\
10 & 0.326515 & 0.327360 & -0.0026 \\
15 & 0.356960 & 0.357500 & -0.0015 \\
20 & 0.375270 & 0.375663 & -0.0010 \\
25 & 0.387370 & 0.387717 & -0.0009 \\
30 & 0.395545 & 0.395872 & -0.0008 \\
35 & 0.400950 & 0.401260 & -0.0008 \\
40 & 0.404285 & 0.404578 & -0.0007 \\
45 & 0.406045 & 0.406317 & -0.0007 \\
50 & 0.406585 & 0.406837 & -0.0006 \\
55 & 0.406185 & 0.406414 & -0.0006 \\
60 & 0.405060 & 0.405261 & -0.0005 \\
65 & 0.403360 & 0.403545 & -0.0005 \\
70 & 0.401235 & 0.401397 & -0.0004 \\
75 & 0.398775 & 0.398917 & -0.0004 \\
80 & 0.396065 & 0.396188 & -0.0003 \\
85 & 0.393160 & 0.393274 & -0.0003 \\
90 & 0.390125 & 0.390224 & -0.0003 \\
95 & 0.386995 & 0.387079 & -0.0002 \\
100 & 0.383800 & 0.383871 & -0.0002 \\
& & & \\
\hline
\end{tabular}

The maximum variation is seen to occur at the initiation of the analysis with TMAP4 underpredicting the concentration by about $0.7 \%$. However, within a short time this difference approaches zero, and the calculation is correct to three significant figures. Error functions were calculated using Lotus $123^{\text {m }}$ and including 26 terms in the series solution for arguments less than 3.0.

At the surface $(x=0)$ the concentration is given by 


$$
C=C_{0} e r f\left(\frac{h}{2 \sqrt{D t}}\right)
$$

while at $x=h$ its value is described by

$$
C=\frac{C_{0}}{2} \operatorname{erf}\left(\frac{h}{\sqrt{D t}}\right)
$$

Tables 16 and 17 list the analytical solutions for Equations (5) and (6) along with their TMAP4-calculated counterparts. Differences between the analytical solutions and the calculated values are virtually non-existent over the length of time being analyzed. Again TMAP4 was run on an IBM PS 2/70. The theory was cvaluated as a series in Lotus $123^{\mathrm{m}}$ discarding terms of order $1.0 \mathrm{E}-14$ and smaller.

TABle 16 Comparison of TMAP4 and ANalytical SOlution FOR Concentration history at $X=0$ in a Partially Preloaded Semi-infinite Slab

\begin{tabular}{rlll}
\hline Time $(\mathrm{s})$ & TMAP4 & Theory & $\begin{array}{c}\text { Variance } \\
0\end{array}$ \\
0 & 1 & 1 & 0.000000 \\
5 & 0.99752 & 0.998435 & -0.000916 \\
10 & 0.9731 & 0.974653 & -0.001593 \\
15 & 0.93114 & 0.932111 & -0.001042 \\
20 & 0.88569 & 0.886154 & -0.000523 \\
25 & 0.84255 & 0.842701 & -0.000179 \\
30 & 0.80333 & 0.803294 & 0.000044 \\
35 & 0.76814 & 0.768002 & 0.000179 \\
40 & 0.73664 & 0.736448 & 0.000261 \\
45 & 0.70839 & 0.708159 & 0.000326 \\
50 & 0.68293 & 0.682689 & 0.000352 \\
55 & 0.65989 & 0.659644 & 0.000373 \\
60 & 0.63893 & 0.63869 & 0.000376 \\
65 & 0.61978 & 0.619545 & 0.000380 \\
70 & 0.60221 & 0.601975 & 0.000390 \\
75 & 0.58601 & 0.585784 & 0.000386 \\
80 & 0.57102 & 0.570805 & 0.000377 \\
85 & 0.55711 & 0.556898 & 0.000381 \\
90 & 0.54414 & 0.543943 & 0.000361 \\
95 & 0.53203 & 0.53184 & 0.000357 \\
100 & 0.52068 & 0.5205 & 0.000346 \\
& & & \\
\hline
\end{tabular}


TABle 17 COMParison OF TMAP4 ANd ANALYTICAL SOLUTION FOR Concentration history at $\mathrm{X}=10 \mathrm{M}$ in a Partially Preloaded Semi-|infinite Slab

\begin{tabular}{cllr}
\hline Time $(s)$ & TMAP4 & Theory & \multicolumn{1}{c}{ Variance } \\
0 & 0.5 & 0.500000 & 0.000000 \\
5 & 0.5 & 0.500000 & 0.000000 \\
10 & 0.49999 & 0.500000 & -0.000020 \\
15 & 0.49982 & 0.499870 & -0.000099 \\
20 & 0.4991 & 0.499217 & -0.000235 \\
25 & 0.49749 & 0.497661 & -0.000344 \\
30 & 0.49489 & 0.495088 & -0.000401 \\
35 & 0.49138 & 0.491586 & -0.000420 \\
40 & 0.48713 & 0.487326 & -0.000403 \\
45 & 0.48231 & 0.482493 & -0.000378 \\
50 & 0.477085 & 0.477250 & -0.000345 \\
55 & 0.471595 & 0.471735 & -0.000296 \\
60 & 0.465935 & 0.466055 & -0.000258 \\
65 & 0.460195 & 0.460295 & -0.000217 \\
70 & 0.45443 & 0.454516 & -0.000188 \\
75 & 0.448695 & 0.448765 & -0.000155 \\
80 & 0.44302 & 0.443077 & -0.000128 \\
85 & 0.43743 & 0.437477 & -0.000106 \\
90 & 0.43195 & 0.431981 & -0.000073 \\
95 & 0.426575 & 0.426603 & -0.000067 \\
100 & 0.42133 & 0.421350 & -0.000048 \\
\hline
\end{tabular}

1d) Permeation Problem with Trapping ${ }^{2}$ (val-1da.inp, val-1db.inp)

This validation problem models permeation through a membrane with a constant source in which traps are operative. The breakthrough time may have one of two limiting values depending on whether the trapping is in the effective diffusivity or strongtrapping regime. ${ }^{3}$ A trapping parameter is defined by

$$
\zeta=\frac{\lambda^{3} v}{\rho D_{0}} \exp \left(\frac{E_{d}-e}{k T}\right)+\frac{c}{\rho}
$$

where

$$
\begin{aligned}
& \lambda=\text { lattice parameter } \\
& \nu=\text { Debye frequency }\left(\approx 10^{13} \mathrm{~s}^{-1}\right)
\end{aligned}
$$


$\rho=$ trapping site fraction

$D_{\mathrm{o}}=$ diffusivity pre-exponential

$E_{\mathrm{d}}=$ diffusion activation energy

$\epsilon=$ trap energy

$k=$ Boltzmann's constant

$T=$ temperature

$c=$ dissolved gas atom fraction.

The descriminant for which regime is dominant is the ratio of $\zeta$ to $c / \rho$. If $\zeta \gg c / \rho$ then the effective diffusivity regime applies, and the permeation transient is identical to the standard diffusion transient but with the diffusivity replaced by an effective diffusivity,

$$
D_{\text {eff }}=\frac{D}{1+\frac{1}{\zeta}} \text {. }
$$

In this limit, the breakthrough time, defined as the intersection of the steepest tangent to the diffusion transient with the time axis, will be

$$
T_{b_{e}}=\frac{l^{2}}{2 \pi^{2} D_{e f f}} .
$$

where $l$ is the thickness of the slab and $D$ is the diffusivity of the gas through the material. The permeation transient is then given by

$$
J_{p}=\frac{c_{0} D}{l}\left\{1+2 \sum_{m=1}^{\dot{L}}\left[(-1)^{m} \exp \left(-2 m^{2} \frac{t}{\tau_{b_{b}}}\right)\right]\right\}
$$

where $\tau_{b}$ is as defined in Equation (9).

In the deep-trapping limit, $\zeta \varangle c / \rho$, and no permeation occurs until essentially all the traps have been filled. Then permeation rapidly turns on to its steady state value. The breakthrough time is given by 


$$
\tau_{b_{d}}=\frac{l^{2} \rho}{2 c_{o} D}
$$

where $c_{0}$ is the steady dissolved gas concentration at the upstream $(\mathrm{x}=0)$ side.

We exercised TMAP4 in both these limits with an upstream-side starting concentration of 0.0001 atom fraction, a diffusivity of $1 \mathrm{~m}^{2} / \mathrm{s}$, a trapping site fraction of $0.1, \lambda^{2}=10^{-15} \mathrm{~m}^{2}$, and a temperature of $1000 \mathrm{~K}$. For the effective diffusivity limit, we selected $\epsilon / k=100 \mathrm{~K}$ to give $\zeta=90.48 \mathrm{c} / \rho$. For the deep trapping limit we took $\epsilon / k=$ $10000 \mathrm{~K}$ to give $\zeta=0.04533 \mathrm{c} / \mathrm{p}$. Aside from the coarser convergence limit required to run the strong-trapping model, there were no other differences in the input files. The results are presented in Figures 4 and 5. 


\section{Effective-Diffusivity Trapping in a Slab}

SQAP Reference 4.2.1.d

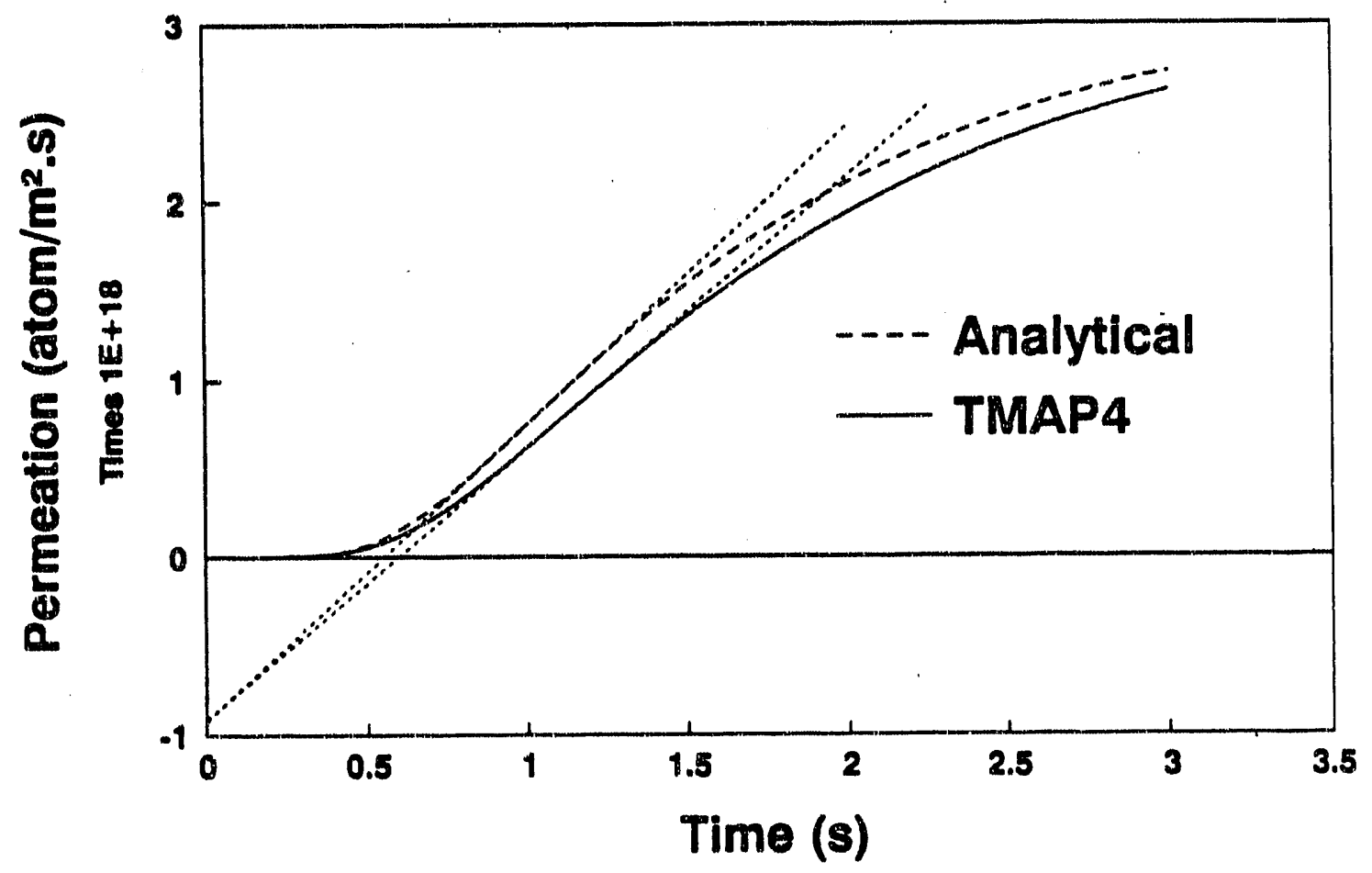

Figure 4. Permeation history for a slab subject to effective-diffusivity limit trapping.

Notice that in neither case is the behavior "ideal" in the sense that the calculated transient differs somewhat from the theoretical one for the limiting case. Such deviation is to be expected because in neither case was the actual problem ideal. In Figure 4 for the effective-diffusivity behavior, the breakthrough time in the calculation is 0.5917 seconds as compared with a theoretical value of 0.5599 seconds. However, the effects of trapping are clearly evident because the breakthrough time in the absence of trapping would have been 0.0506 seconds for the same parameters otherwise. In Figure 5 note that the permeation turns on much more abruptly than in Figure 4, and it agrees quite well with the breakthrough time of 500 seconds calculated by Equation (11). There is some diffusive permeation before the theoretical breakthrough time and it takes a while 


\section{Strong Trapping in a Slab SQAP Reference 4.2.1.d}

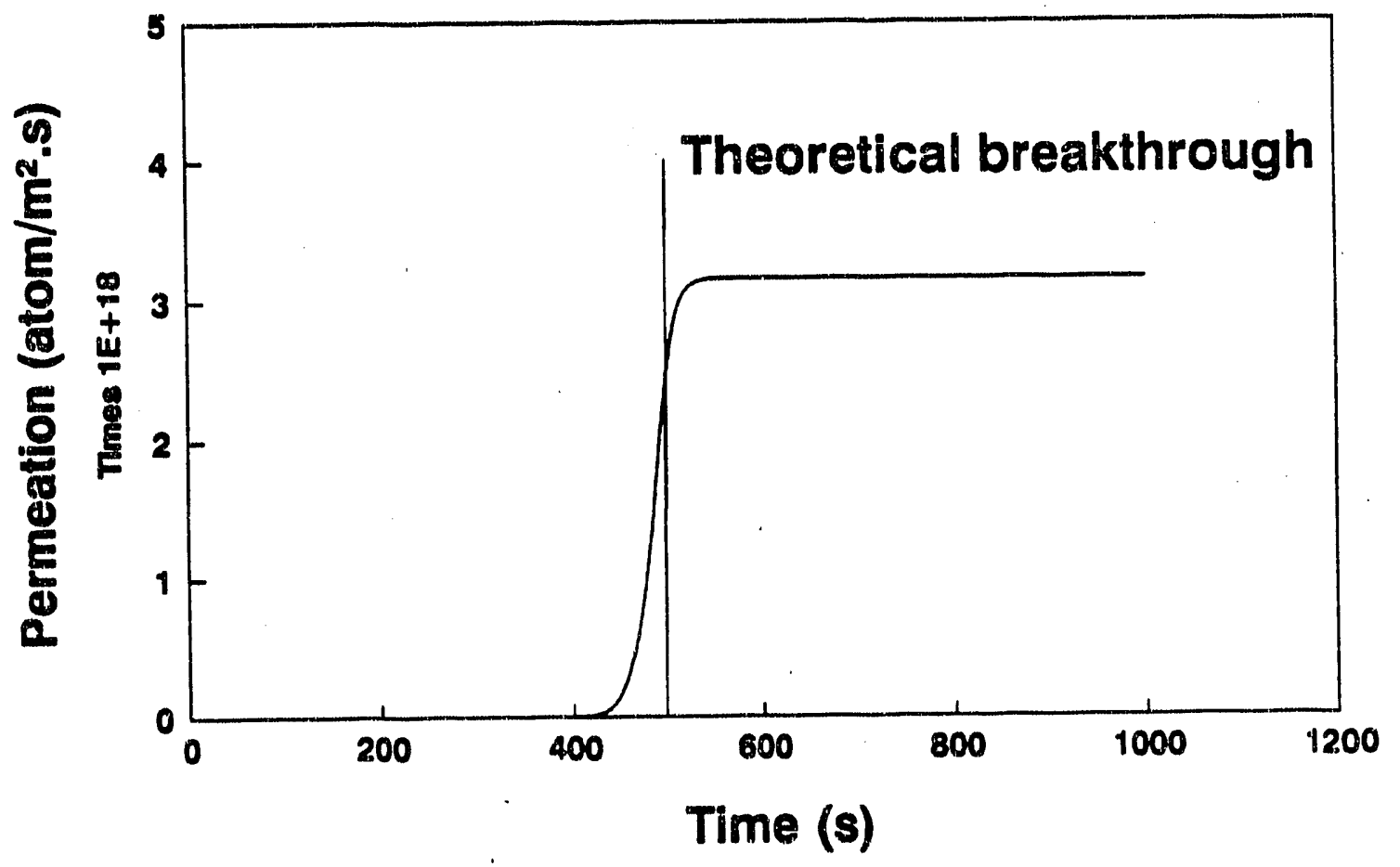

Figure 5. Permeation transient in a slab subject to strong-trapping.

to saturate after breakthrough. However, the general characteristics in both cases are in good agreement with the theory.

1e) Diffusion Problem with Composite Material Layers ${ }^{2}$ (val-1e.inp)

A composite layer of $\mathrm{PyC}$ and $\mathrm{SiC}$ is modelled with a constant concentration boundary condition on the surface of the PyC. The concentration in the second layer (SiC) of this composite can be found from the following expression 


$$
\begin{aligned}
C=C_{o} \frac{D_{A y C}(a-x)}{a D_{P Y C}+l D_{S i C}} & \\
& -2 C_{o} \sum_{n=1}^{\infty} \frac{\sin \left(l \beta_{n}\right) \sin \left(k a \beta_{n}\right) \sin \left[k(a-x) \beta_{n}\right]}{\beta_{n}\left[l \sin ^{2}\left(k a \beta_{n}\right)+\sigma k a \sin ^{2}\left(l \beta_{n}\right)\right]} \exp \left(-D_{P y C} \beta_{n}^{2} t\right)
\end{aligned}
$$

where

$$
\begin{aligned}
& a=\text { thickness of PyC } \\
& l=\text { thickness of } \mathrm{SiC} \\
& k=\sqrt{\frac{D_{P y C}}{D_{S i C}}} \\
& \sigma=\frac{1}{k}
\end{aligned}
$$

and $\beta_{\mathrm{n}}$ are the roots of

$$
\cot (\beta l)+\sigma \cot (k \beta a)=0 \text {. }
$$

The TMAP4 calculated concentration for $\mathrm{x}=8 \mu \mathrm{m}$ is compared to the solution given by Equation (12) for a case where $a=33 \mu \mathrm{m}, l=63 \mu \mathrm{m}$ in Table 18. The agreement is very good, although TMAP4 underpredicts slightly at short times. The agreement is better than $0.1 \%$ over most of the interval of comparison and is better than $5 \%$ everywhere. The same comparison is made graphically in Figure 6. 
TABle 18 Comparison of TMAP4 and ANalytical Solution for CONCENTRATION History (ATOM/M $\left.\mathrm{M}^{3}\right)$ at $\mathrm{X}=8$ MICROMETERS IN A COMPOSITE MATERIAL

$\begin{array}{clll}\text { Time }(\mathrm{s}) & \text { TMPA4 } & \text { Theory } & \text { Variance } \\ 0 & 0 & 0 & 0 \\ 5 & 1.80950 \mathrm{e}+25 & 1.89284 \mathrm{e}+25 & -0.04403 \\ 10 & 2.14600 \mathrm{e}+25 & 2.18703 \mathrm{e}+25 & -0.01876 \\ 15 & 2.25380 \mathrm{e}+25 & 2.27425 \mathrm{e}+25 & -0.00899 \\ 20 & 2.29160 \mathrm{e}+25 & 2.30083 \mathrm{e}+25 & -0.00401 \\ 25 & 2.30510 \mathrm{e}+25 & 2.30894 \mathrm{e}+25 & -0.00166 \\ 30 & 2.30990 \mathrm{e}+25 & 2.31142 \mathrm{e}+25 & -0.00066 \\ 35 & 2.31160 \mathrm{e}+25 & 2.31217 \mathrm{e}+25 & -0.00025 \\ 40 & 2.31220 \mathrm{e}+25 & 2.31240 \mathrm{e}+25 & -0.00009 \\ 45 & 2.31240 \mathrm{e}+25 & 2.31247 \mathrm{e}+25 & -0.00003 \\ 50 & 2.31250 \mathrm{e}+25 & 2.31249 \mathrm{e}+25 & 0.00000\end{array}$

\section{Diffusion in Composite Material Layers SQAP Reference 4.2.1.e}

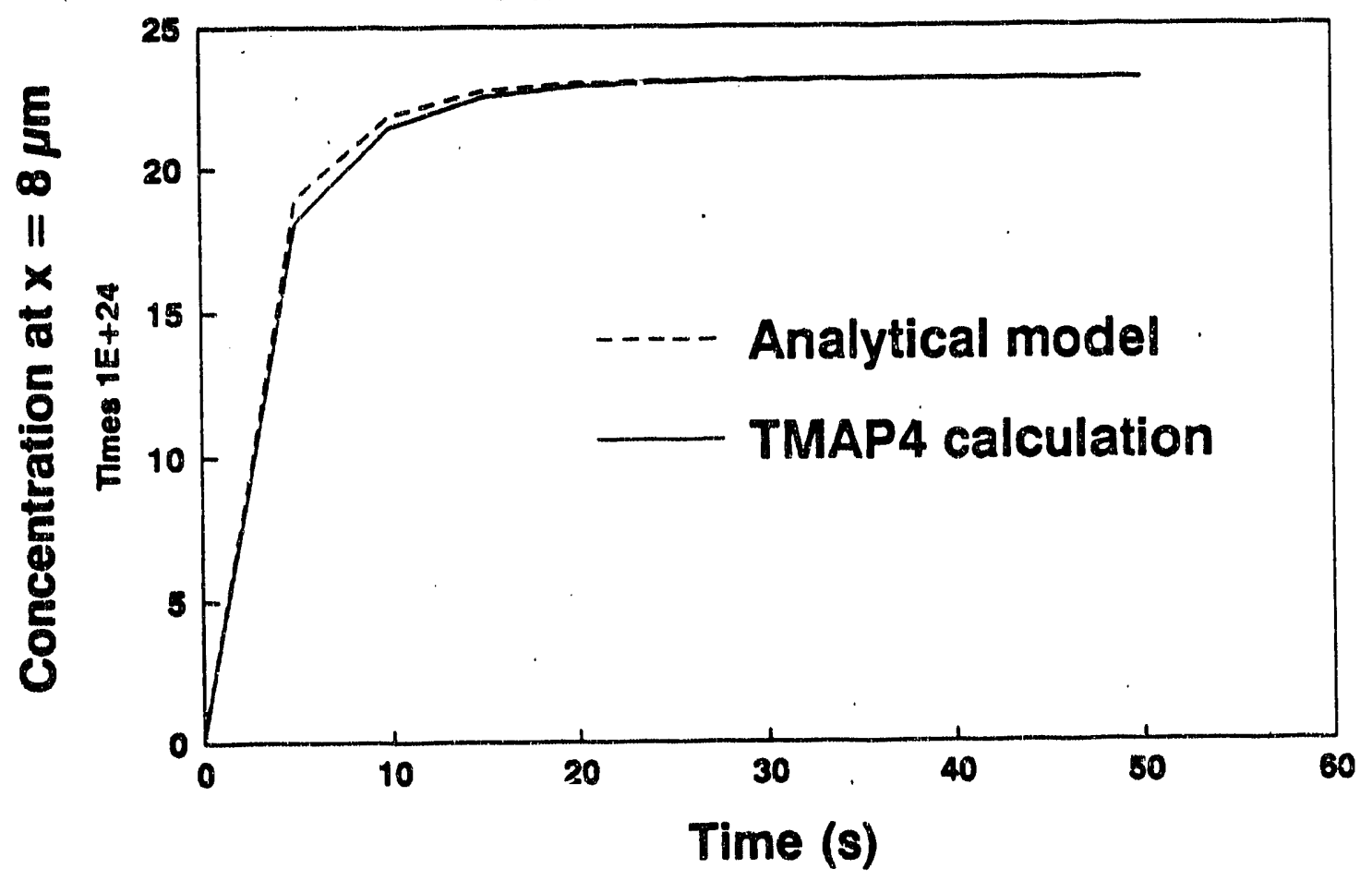

Figure 6. Comparison of TMAP4 results with theory for concentration build-up by diffusion in a composite-layer material with a constant source on one side. 
1f) Heat Sink/Source Problem (val-1fa.inp, val-1fb.inp)

The heat transfer calculation capability of the TMAP4 code is validated using two cases. The first case examines TMAP4's thermal capabilities by modelling heat conduction in a slab having internal heat generation. An adiabatic surface is applied to one side of the layer while a constant temperature is imposed on the other surface. The analytical solution for this case is given by

$$
T=T_{s}+\frac{Q L^{2}}{2 k}\left(1-\frac{x^{2}}{L^{2}}\right)
$$

where

$Q=$ internal heat generation rate

$L=$ thickness of the layer

$\boldsymbol{x}=$ thermal conductivity

$T_{\mathrm{s}}=$ imposed surface temperature.

Table 19 compares the calculated temperature as a function of position between TMAP4 results and Equation (13) for $Q=1.0 \times 10^{4} \mathrm{~W} / \mathrm{m}^{3}, L=1.6 \mathrm{~m}, \mathrm{x}=10 \mathrm{~W} / \mathrm{m} \cdot \mathrm{K}$, and $T_{\mathrm{s}}=300 \mathrm{~K}$. We also use $\rho \mathrm{C}_{\mathrm{p}}=1 \mathrm{~J} / \mathrm{m}^{3} \cdot \mathrm{K}$, for a thermal time constant of $26 \mathrm{~ms}$, and

TABle 19 COMPARISON OF TMAP4 AND ANALYTICAL SOLUTION FOR Heat Sink/Source Problem with Internal heat Generation

\begin{tabular}{cccl}
\hline Position (m) & TMAP4 & Theory & Variance \\
0 & 1580 & 1580 & 0 \\
0.1 & 1580 & 1575 & 0.003175 \\
0.3 & 1540 & 1535 & 0.003257 \\
0.5 & 1460 & 1455 & 0.003436 \\
0.7 & 1340 & 1335 & 0.003745 \\
0.9 & 1180 & 1175 & 0.004255 \\
1.1 & 980 & 975 & 0.005128 \\
1.3 & 740 & 735 & 0.006803 \\
1.5 & 460 & 455 & 0.010989 \\
1.6 & 300 & 300 & 0 \\
\hline
\end{tabular}


an elapsed time of 50 seconds. At that time steady state has been reached. As may be seen in Table 19, the agreement is excellent.

In a second case an effective mass diffusivity is used to simulate a thermal transient. In that instance temperature is held constant, but the mass-diffusion transient is taken as an analog for the thermal transient. The same geometry is assumed, except that the thickness, $L$, is only $0.375 \mathrm{~m}$. The general equation is

$$
T(x, t)=T_{0}+\left(T_{1}-T_{0}\right)\left[1-\frac{2}{\pi} \sum_{m=0}^{\infty} \frac{(-1)^{m}}{\left(m+\frac{1}{2}\right)} \cos \left(\gamma_{m} x\right) \exp \left(-\alpha \gamma_{m}^{2} t\right)\right]
$$

where

$$
\gamma_{m}=\left(m+\frac{1}{2}\right) \frac{\pi}{L}
$$

and

$$
\alpha=\frac{k}{\rho C_{p}}
$$

is the thermal diffusivity. By substituting temperatures for concentrations and the thermal diffusivity for the mass diffusivity, we complete the analog solution. Here, $\alpha=1.29035 \times 10^{-3} \mathrm{~m}^{2} / \mathrm{s}, T_{\mathrm{o}}=300 \mathrm{~K}$, and $T_{1}=373 \mathrm{~K}$. Table 20 compares the TMAP4 results at $x=0$ with those of Equation (14). Again, the agreement is very good.

1g) Simple Chemical Reaction Problem (val-1ga.inp, val-1gb.inp)

A simple time-dependent chemical reaction given by

$$
A+B \rightarrow A B
$$

is modelled in a functional enclosure. The reaction rate, $R_{\mathrm{c}}$, is positive if the species $A B$ is being produced in the reaction and negative if it is being consumed. The forward rate coefficient, $K_{\mathrm{R}}$, for the reaction has no spatial or time dependence. The reaction rate is 
TABle 20 Comparison on tMaP4 and ANalytical Results fOR Simulation of a Thermal Transient by a Mass-Diffusion Transient

\begin{tabular}{clll}
\hline Time $(s)$ & TMAP4 & Theory & Variance \\
0 & 300 & 300 & 0 \\
10 & 305.4 & 302.8584 & 0.008392 \\
20 & 314.25 & 314.427 & -0.00056 \\
30 & 323.69 & 325.9433 & -0.00691 \\
40 & 332.28 & 335.4314 & -0.0094 \\
50 & 339.62 & 343.037 & -0.00996 \\
60 & 345.72 & 349.1069 & -0.0097 \\
70 & 350.73 & 353.9476 & -0.00909 \\
80 & 354.83 & 357.8077 & -0.00832 \\
90 & 358.18 & 360.8857 & -0.0075 \\
100 & 360.92 & 363.3401 & -0.00666 \\
110 & 363.15 & 365.2973 & -0.00588 \\
120 & 364.96 & 366.8579 & -0.00517 \\
130 & 366.45 & 368.1023 & -0.00449 \\
140 & 367.65 & 369.0946 & -0.00391 \\
150 & 368.64 & 369.8858 & -0.00337 \\
160 & 369.45 & 370.5168 & -0.00288 \\
170 & 370.1 & 371.0199 & -0.00248 \\
180 & 370.64 & 371.4211 & -0.0021 \\
\hline
\end{tabular}

$$
R_{c}=K_{r} C_{A} C_{B}
$$

The analytical solution for the concentration of species $A B$ is the function

$$
C_{A B}=C_{B_{0}} \frac{1-\exp \left[K_{R} t\left(C_{B_{0}}-C_{A_{0}}\right)\right]}{1-\frac{C_{B_{0}}}{C_{A_{0}}} \exp \left[K_{R} t\left(C_{B_{0}}-C_{A_{0}}\right)\right]}
$$

where

$$
\begin{aligned}
& C_{A B}=\text { concentration of species } A B \\
& C_{A_{0}}=\text { initial concentration of species } A \\
& C_{B_{0}}=\text { initial concentiation of species } B
\end{aligned}
$$

In the special case when $C_{A_{0}}$ and $C_{B_{0}}$ are equal, this becomes 


$$
C_{A B}=C_{A_{0}}-\frac{1}{\frac{1}{C_{A_{0}}}+K_{R} t}
$$

We exercised TMAP4 under each of these conditions. Table 21 gives a numerical comparison of the values computed by TMAP4 when the starting pressure of species $A$ and $B$ were both $1.0 \mu \mathrm{Pa}$, and $K_{\mathrm{R}}$ was $4.14 \times 10^{-15}$ molecule. $\mathrm{m}^{3} / \mathrm{atom}^{2} . \mathrm{s}$. The calculation and theoretical value are in excellent agreement. Table 22 gives the comparison for the same input file but with $C_{B_{0}}$ reduced to $0.1 \mu \mathrm{Pa}$. These are shown graphically in Figure 7. The distinction between the TMAP4 values and the analytical results vanishes.

\section{Simple Chemical Reaction Problem}

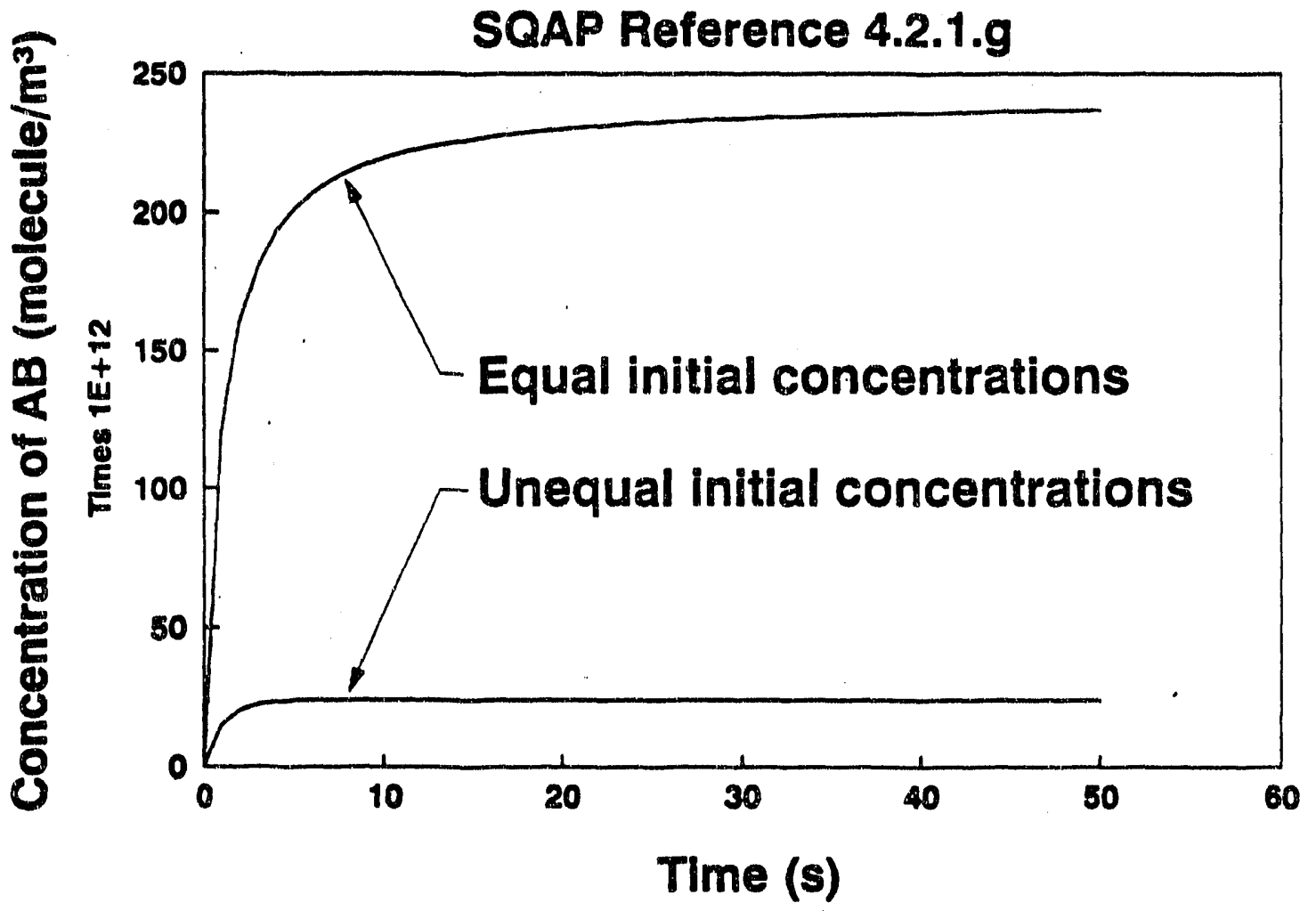

Figure 7. TMAP4 calculations agreed well with theoretical values for both equal and unequal starting reactant concentrations. 
TABle 21 Comparison of TMAP4 and ANAlytical Results fOR Simple Chemical Reaction Problem with Equal Starting Concentrations

\begin{tabular}{|c|c|c|c|}
\hline Time (s) & TMAP4 & Theory & Variance \\
\hline 0 & 0 & 0 & 0 \\
\hline 1 & $1.2036 \mathrm{e}+14$ & $1.2077 \mathrm{e}+14$ & -0.0034186 \\
\hline 2 & $1.6074 \mathrm{e}+14$ & $1.6103 e+14$ & -0.0018041 \\
\hline 3 & $1.8095 e+14$ & $1.8116 \mathrm{e}+14$ & -0.0011555 \\
\hline 4 & $1.9308 e+14$ & $1.9324 \mathrm{e}+14$ & -0.0008105 \\
\hline 5 & $2.0117 e+14$ & $2.0129 e+14$ & -0.000587 \\
\hline 6 & $2.0694 e+14$ & $2.0704 e+14$ & -0.0004794 \\
\hline 7 & $2.1127 e+14$ & $2.1135 \mathrm{e}+14$ & -0.0003906 \\
\hline 8 & $2.1464 \mathrm{e}+14$ & $2.1471 e+14$ & -0.0003138 \\
\hline 9 & $2.1734 \mathrm{e}+14$ & $2.1739 \mathrm{e}+14$ & -0.0002356 \\
\hline 10 & $2.1954 e+14$ & $2.1959 e+14$ & -0.0002144 \\
\hline 11 & $2.2138 e+14$ & $2.2142 e+14$ & -0.000167 \\
\hline 12 & $2.2293 e+14$ & $2.2297 e+14$ & -0.0001585 \\
\hline 13 & $2.2426 \mathrm{e}+14$ & $2.2429 \mathrm{e}+14$ & -0.000145 \\
\hline 14 & $2.2541 e+14$ & $2.2544 \mathrm{e}+14$ & -0.0001452 \\
\hline 15 & $2.2642 \mathrm{e}+14$ & $2.2645 \mathrm{e}+14$ & -0.0001289 \\
\hline 16 & $2.2731 e+14$ & $2.2734 \mathrm{e}+14$ & -0.0001197 \\
\hline 17 & $2.2811 e+14$ & $2.2813 e+14$ & -0.0000727 \\
\hline 18 & $2.2881 e+14$ & $2.2883 e+14$ & -0.0000999 \\
\hline 19 & $2.2945 e+14$ & $2.2947 e+14$ & -0.0000806 \\
\hline 20 & $2.3003 e+14$ & $2.3004 \mathrm{e}+14$ & -0.0000592 \\
\hline 21 & $2.3055 e+14$ & $2.3057 e+14$ & -0.0000713 \\
\hline 22 & $2.3103 e+14$ & $2.3104 e+14$ & -0.0000597 \\
\hline 23 & $2.3147 e+14$ & $2.3148 \mathrm{e}+14$ & -0.0000492 \\
\hline 24 & $2.3187 e+14$ & $2.3188 e+14$ & -0.0000602 \\
\hline 25 & $2.3224 \mathrm{e}+14$ & $2.3226 \mathrm{e}+14$ & -0.0000671 \\
\hline 26 & $2.3259 e+14$ & $2.3260 \mathrm{e}+14$ & -0.0000415 \\
\hline 27 & $2.3291 e+14$ & $2.3292 \mathrm{e}+14$ & -0.0000393 \\
\hline 28 & $2.3321 e+14$ & $2.3322 \mathrm{e}+14$ & -0.0000284 \\
\hline 29 & $2.3349 e+14$ & $2.3349 e+14$ & -0.0000183 \\
\hline 30 & $2.3375 e+14$ & $2.3375 e+14$ & -0.0000171 \\
\hline 31 & $2.3399 e+14$ & $2.3400 \mathrm{e}+14$ & -0.000032 \\
\hline 32 & $2.3422 e+14$ & $2.3423 e+14$ & -0.0000266 \\
\hline 33 & $2.3443 e+14$ & $2.3444 e+14$ & -0.0000491 \\
\hline 34 & $2.3464 \mathrm{e}+14$ & $2.3464 \mathrm{e}+14$ & -0.0000191 \\
\hline 35 & $2.3483 e+14$ & $2.3484 e+14$ & -0.0000264 \\
\hline 36 & $2.3501 e+14$ & $2.3502 \mathrm{e}+14$ & -0.0000321 \\
\hline 37 & $2.3518 \mathrm{e}+14$ & $2.3519 e+14$ & -0.0000397 \\
\hline 38 & $2.3535 e+14$ & $2.3535 e+14$ & -0.0000098 \\
\hline 39 & $2.3550 e+14$ & $2.3551 \mathrm{e}+14$ & -0.0000304 \\
\hline 40 & $2.3565 e+14$ & $2.3565 e+14$ & -0.0000188 \\
\hline
\end{tabular}


TABle 22 Comparison of TMÁP4 and ANALYTTCAL RESUlts FOR Simple Chemical Reaction Problem with Unequal Starting Concentrations

\begin{tabular}{clll}
\hline Tine $(s)$ & TMAP4 & Theory & Variance \\
0 & 0 & 0 & 0 \\
1 & $1.4897 \mathrm{e}+13$ & $1.4942 \mathrm{e}+13$ & -0.0029806 \\
2 & $2.0468 \mathrm{e}+13$ & $2.0501 \mathrm{e}+13$ & -0.0015964 \\
3 & $2.2664 \mathrm{e}+13$ & $2.2684 \mathrm{e}+13$ & -0.0008687 \\
4 & $2.3549 \mathrm{e}+13$ & $2.3559 \mathrm{e}+13$ & -0.0004227 \\
5 & $2.3908 \mathrm{e}+13$ & $2.3913 \mathrm{e}+13$ & -0.0002012 \\
6 & $2.4054 \mathrm{e}+13$ & $2.4056 \mathrm{e}+13$ & -0.0000976 \\
7 & $2.4113 \mathrm{e}+13$ & $2.4115 \mathrm{e}+13$ & -0.0000685 \\
8 & $2.4138 \mathrm{e}+13$ & $2.4138 \mathrm{e}+13$ & -0.0000144 \\
9 & $2.4148 \mathrm{e}+13$ & $2.4148 \mathrm{e}+13$ & 0.00000078 \\
10 & $2.415 \mathrm{e}+13$ & $2.4152 \mathrm{e}+13$ & 0.00000426 \\
11 & $2.4153 \mathrm{e}+13$ & $2.4153 \mathrm{e}+13$ & -0.0000203 \\
12 & $2.4154 \mathrm{e}+13$ & $2.4154 \mathrm{e}+13$ & -0.0000057 \\
13 & $2.4154 \mathrm{e}+13$ & $2.4154 \mathrm{e}+13$ & -0.0000165 \\
14 & $2.4155 \mathrm{e}+13$ & $2.4155 \mathrm{e}+13$ & 0.00002042 \\
15 & $2.4155 \mathrm{e}+13$ & $2.4155 \mathrm{e}+13$ & 0.00001862 \\
16 & $2.4155 \mathrm{e}+13$ & $2.4155 \mathrm{e}+13$ & 0.00001789 \\
17 & $2.4155 \mathrm{e}+13$ & $2.4155 \mathrm{e}+13$ & 0.00001759 \\
18 & $2.4155 \mathrm{e}+13$ & $2.4155 \mathrm{e}+13$ & 0.00001747 \\
19 & $2.4155 \mathrm{e}+13$ & $2.4155 \mathrm{e}+13$ & 0.00001742 \\
20 & $2.4155 \mathrm{e}+13$ & $2.4155 \mathrm{e}+13$ & 0.0000174 \\
21 & $2.4155 \mathrm{e}+13$ & $2.135 \mathrm{e}+13$ & 0.00001739 \\
22 & $2.4155 \mathrm{e}+13$ & $2.4155 \mathrm{e}+13$ & 0.00001739 \\
23 & $2.4155 \mathrm{e}+13$ & $2.4155 \mathrm{e}+13$ & 0.00001739 \\
24 & $2.4155 \mathrm{e}+13$ & $2.4155 \mathrm{e}+13$ & 0.00001739 \\
25 & $2.4155 \mathrm{e}+13$ & $2.4155 \mathrm{e}+13$ & 0.00001739 \\
\hline
\end{tabular}

1h) System (Multiple Enclosure Volumes) Problem (val-1h.inp)

A three-enclosure model is presented having a known convective outflow from one enclosure to the next. The first enclosure is a boundary enclosure with a constant concentration of the species " $t 2$ ". The flux, $\bar{j}$, of molecules into the first functional enclosure (number 2 ) is given by 


$$
\bar{j}=Q C_{1}
$$

where

$$
\begin{aligned}
& Q=\text { volumetric flow rate, common through the enclosures } \\
& C_{1}=\text { constant concentration in enclosure } 1 .
\end{aligned}
$$

Gas from the first enclosure flows into the second and ultimately into the third with both enclosures 2 and 3 having initial concentrations of zero. Concentrations of particles in both functional enclosures 2 and 3 are found as a function of time. The analytical solution consists of the two simultaneous equations:

$$
\begin{aligned}
& \frac{\partial C_{2}}{\partial t}=\frac{Q\left(C_{1}-C_{2}\right)}{V_{2}} \\
& \frac{\partial C_{3}}{\partial t}=\frac{Q\left(C_{2}-C_{3}\right)}{V_{3}}
\end{aligned}
$$

Solutions to this set with the initial condition that $\dot{C}_{2_{0}}=C_{3_{0}}=0$ are

$$
C_{2}=C_{1}\left[1-\exp \left(-\frac{Q t}{V_{2}}\right)\right]
$$

and

$$
C_{3}=C_{1}\left[1-\left(1+\frac{Q t}{V}\right) \exp \left(\frac{Q t}{V}\right)\right]
$$

if $V_{2}=V_{3}=V$ or

$$
C_{3}=C_{1}\left[1-\frac{V_{2}}{V_{2}-V_{3}} \exp \left(-\frac{Q t}{V_{2}}\right)+\frac{V_{3}}{V_{2}-V_{3}} \exp \left(-\frac{Q t}{V_{3}}\right)\right]
$$

otherwise. These equations were solved using Lotus $123^{\mathrm{m}}$ for volumes of $1 \mathrm{~m}^{3}$ for each enclosure, and an inlet "t2" partial pressure of $1 \mathrm{~Pa}$. Graphical comparison of results is given in Figure 8 where it is impossible to distinguish between the TMAP4 calculation 
and the theoretical result. The data are listed along with variances between TMAP4 and Lotus solutions in Table 23.

\section{Multiple Enclosure Charnber Problem SQAP Reference 4.2.1.h}

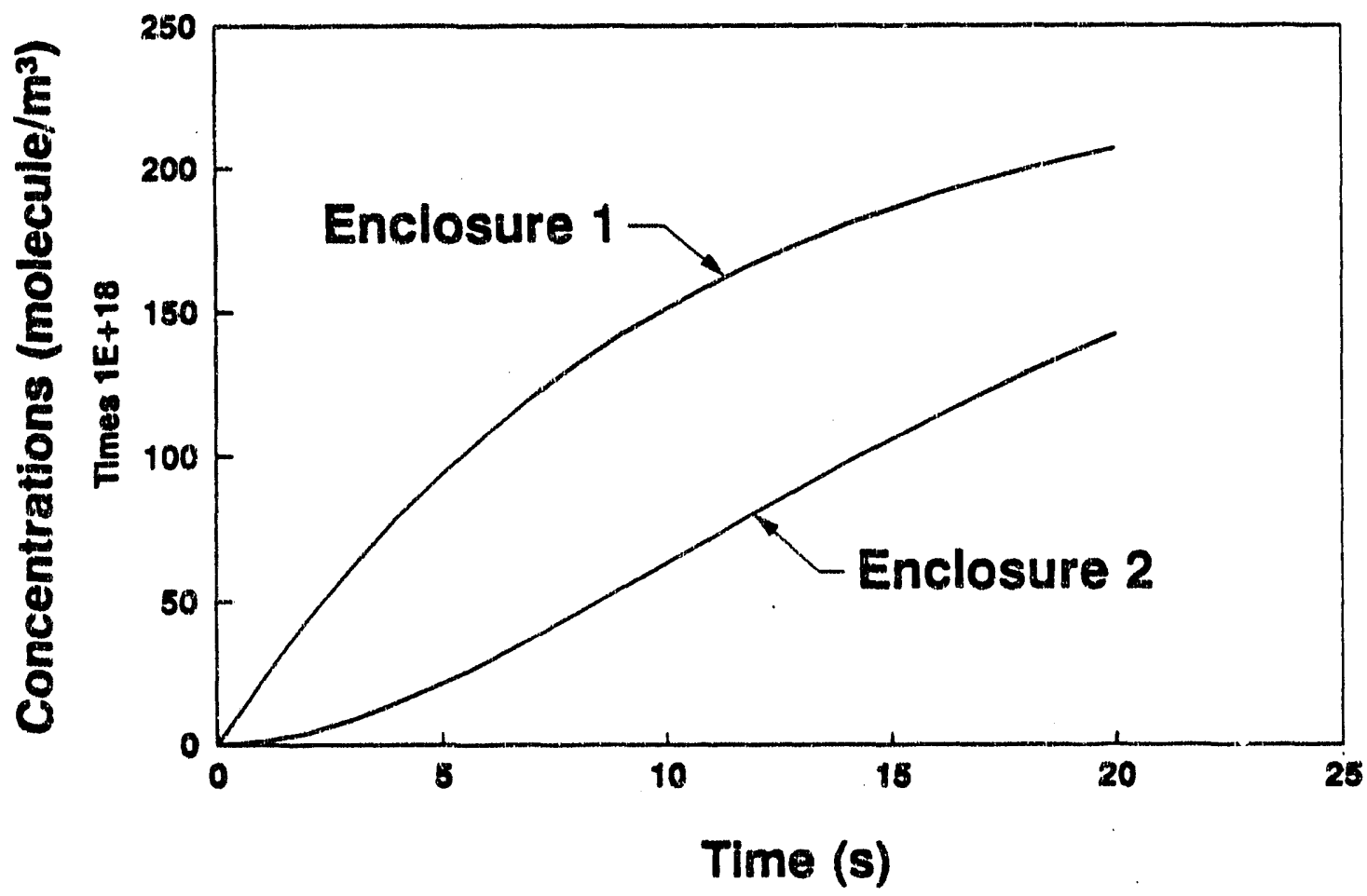

Figure 8. There is virtually no difference between TMAP4 calculations and analytical results for the case of concentration transients in multiple chambers. 


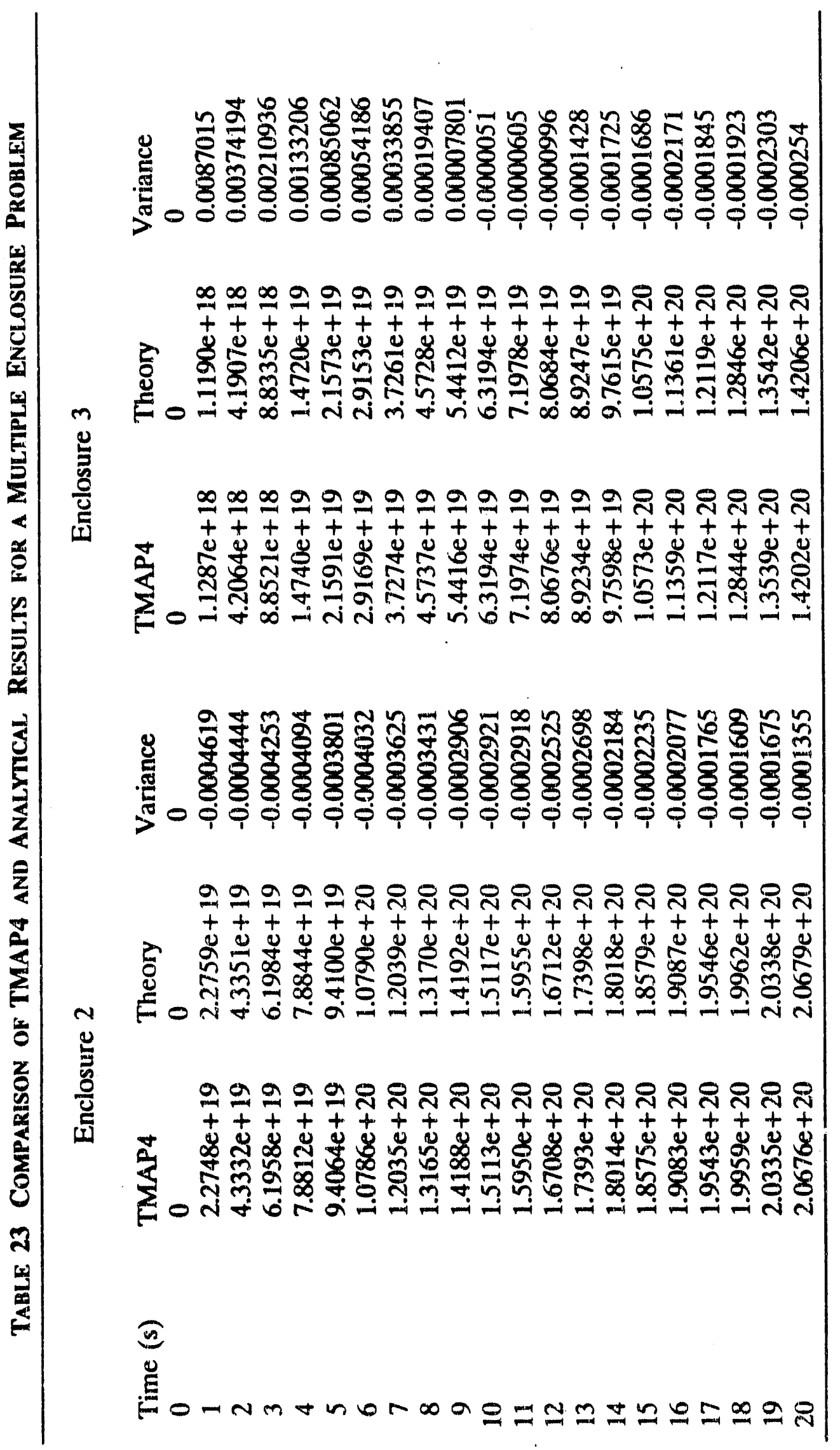




\section{Comparison with Experiments}

The second phase of code validation is the comparison of code results with actual experimental data. Three experimental data sets were selected for modelling. Each was published in a refereed journal.

\section{2a) Ion Implantation Experiment (val-2a.inp)}

This problem is the simulation of experimental results obtained at the INEL in 1985 and published. ${ }^{4}$ The experiment involved applying an ion beam to a $2.5-\mathrm{cm}$ diameter, $0.5-\mathrm{mm}$ thick sample of a modified 316 stainless steel called Primary Candidate Alloy (PCA). Details of the experiment and the means of evaluating the necessary transport parameters to get a good fit between TMAP4 results and the experimental data are given in the publication. The TRIM code was used to determine that the average implantation depth for the ions was $11 \mu \mathrm{m} \pm 5.4 \mu \mathrm{m}$. Reemission data from the TRIM calculation showed that only $75 \%$ of the incident flux remained in the metal. The other $25 \%$ was re-emitted.

One known non-physical feature in the modelling is that the cleanup of the upstream surface was modelled by a simple exponential in time rather than in ion fluence which was interrupted twice during the actual experiment. The pressures upstream and downstream could have been taken as zero and obtained essentially the same results.

The plot of Figure 9 was generated. Actual experimental data are also shown on the figure. They are fairly closely approximated by the calculated permeation. Notice in the figure, however that there is a lower permeation flux value when the beam is on, and a relatively slow trail-off, compared with the calculation, when the beam was turned off. Some of this is a consequence of the experimental technique where the walls of the experimental chamber did some pumping of the gas as it came through the sample and then provided a source of deuterium when the sample permeation ceased. Some twodimensional effects also influence the comparison. 


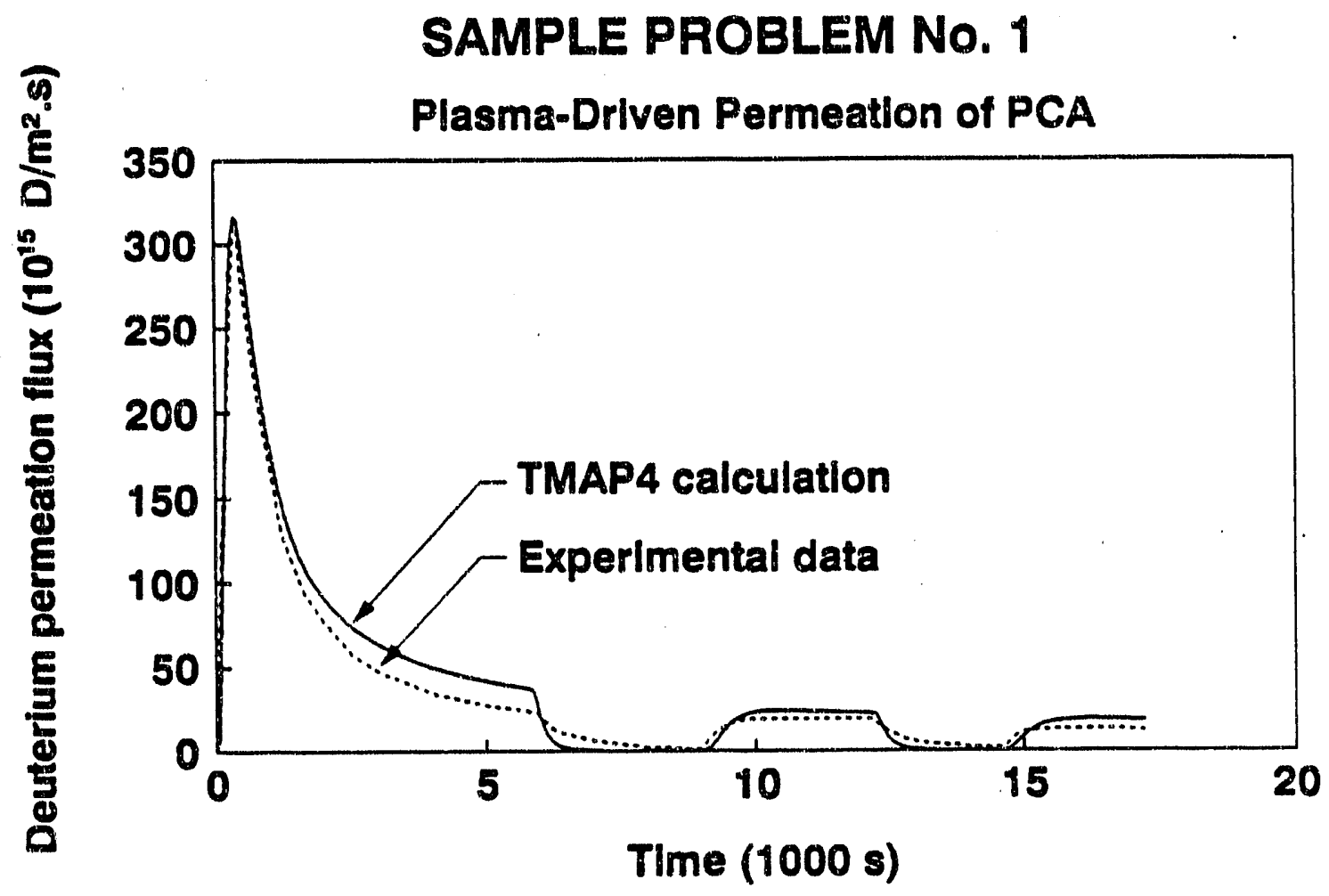

Figure 9. Computed permeation transient which is very similar to experimental data from an ion-implantation experiment.

2b) Material Diffusion Experiment (val-2ba.inp, val2bb.inp)

This problem is taken from work done by R. G. Macaulay-Newcombe at McMaster University. ${ }^{5} \mathrm{He}$ and co-workers conducted thermal absorption and desorption experiments, as well as implantation experiments, on wafers of polished beryllium. Of the several data sets presented, the one modelled here is that represented in Figure 2 (a) in their publication. The beryllium was $0.4-\mathrm{mm}$ thick and had an area of $104 \mathrm{~mm}^{2}$. It was polished to a mirror finish then exposed to $13.3 \mathrm{KPa}$ of deuterium at $773 \mathrm{~K}$ for 50 minutes. It was quickly cooled under a vacuum of about $1 \mu \mathrm{Pa}$. The cooling time constant for the apparatus is taken as 45 minutes. After removing the sample from the 
charging furnace, it was transferred in the air to a thermal desorption furnace where the temperature was increased from ambient $(300 \mathrm{~K})$ to $1073 \mathrm{~K}$ at the rate of $3 \mathrm{~K} / \mathrm{min}$. This was done under vacuum, and the pressure of the chamber was monitored by residual gas analysis and calibrated against standard leaks. In that way the emission rate from the sample could be measured as a function of temperature. Data from that measurement, given in Figure 2 (a) of their paper are reproduced in Figure 10. From Rutherford backscattering measurements made on the samples before charging with deuterium they deduced that the thickness of the oxide film was $18 \mathrm{~nm}$. This is typical for polished beryllium. The metal is so reactive in air that the film forms almost immediately after any surface oxide removal. On the other hand, it is relatively stable and would only grow slightly when exposed to air between charging and thermal desorption.

This experiment is modelled using a two-segment model in TMAP4 with the segments linked. The first is the $\mathrm{BeO}$ film, which is modelled using 18 equally spaced nodes of $1 \mathrm{~nm}$ each plus the two surface nodes. The second segment is. a half-thickness wafur of beryllium with reflective boundary conditions at the midplane. It is made up of 15 segments of varying thickness to accommodate solution stiffness plus the two surface nodes. The solubility of deuterium in beryllium used was that given by $\mathrm{K}$. L. Wilson, et al. ${ }^{6}$ based on work done by W. A. Swansiger, also of Sandia National Laboratory. The diffusivity of deuterium in beryllium was measured by E. Abramov, et al. ${ }^{7}$ They made measurements on high-grade ( $99 \%$ pure) and extra grade (99.8\% pure). The values for high-grade, consistent with Dr. Macaulay-Newcombe's measurements of the purity of his samples.

Deuterium transport properties for the $\mathrm{BeO}$ are more challenging. First, it is not clear in what state the deuterium exists in the BeO. However, it has been observed ${ }^{8}$ that an activation energy of $.78 \mathrm{~kJ} /$ mole (exothermic solution) is evident for tritium coming out of neutron irradiated beryllium in work by D. L. Baldwin of Battelle Pacific Northwest Laboratory. The same energy has appeared in other results (can be inferred from Dr. Swansiger's work cited by Wilson, et al. ${ }^{6}$, and by R. A. Causey, et al. ${ }^{9}$, among 
others), so one may be justified in using it. The solubility coefficient is not well known. Measurements reported by R. G. Macaulay-Newcombe, et al. ${ }^{10}$ and in followup telephone conversations indicate about 200 appm of $\mathrm{D}$ in $\mathrm{BeO}$ after exposure to $13.3 \mathrm{kPa}$ of $D_{2}$ at $773 \mathrm{~K}$. That suggests a coefficient of only $1.88 \times 10^{18} \mathrm{~d} / \mathrm{m}^{3} \cdot \mathrm{Pa}^{1 / 2}$. On the other hand, the integrated area under the curve in the referenced experimental data is $1.1 \times 10^{14}$ $\mathrm{d} / \mathrm{mm}^{2}$ which implies a solubility coefficient of $2.85 \times 10^{20} \mathrm{~d} / \mathrm{m}^{3} \cdot \mathrm{Pa}^{1 / 2}$. Since much of the deuterium in the oxide layer will get out during the cool-down process (and because it gives a good fit) the solubility coefficient is taken to be $5 \times 10^{20} \mathrm{~d} / \mathrm{m}^{3} \cdot \mathrm{Pa}^{1 / 2}$.

Deuterium diffusion measurements in $\mathrm{BeO}$ were made by J. D. Fowler, et al. ${ }^{11}$ They found a wide range of results for diffusivity in $\mathrm{BeO}$, depending on the physical form of the material, having measured it for single-crystal, sintered, and powdered $\mathrm{BeO}$. This model uses one expression for the charging phase and another for the thermal desorption phase, believing that the surface film changed somewhat during the transfer between the two furnaces. For the charging phase the model uses 20 times that for the sintered $\mathrm{BeO}$. Thermal expansion mismatches tend to open up cracks and channels in the oxide layer, so this seems a reasonable value. The same activation energy of $48.5 \mathrm{~kJ} / \mathrm{mole}$, is retained, however. For the thermal desorption phase, the prefactor of the sintered material $\left(7 \times 10^{-5} \mathrm{~m}^{2} / \mathrm{s}\right)$ and an activation energy of $223.7 \mathrm{~kJ} / \mathrm{mole}(53.45 \mathrm{kcal} / \mathrm{mole})$ are used. These values give good results and lie well within the scatter of Fowler's data. Exposure of the sample to air after heating should have made the oxide more like single crystal by healing the cracks that may have developed.

The model applies $13.3 \mathrm{kPa}$ of $\mathrm{D}_{2}$ for 50 hours followed by evacuation to $1 \mu \mathrm{Pa}$ and cool down with a 45 minute time constant for one hour. The deuterium concentrations in the sample are of a complex distribution that results from first charging the sample and then discharging it during the cooldown. This problem is then restarted with different equations to simulate thermal desorption in the $1-\mu \mathrm{Pa}$ environment. That begins at $300 \mathrm{~K}$ and goes to $1073 \mathrm{~K}$. Again, the concentration profiles in both the substrate beryllium and the oxide film have a peculiar interaction because of the activation energies involved, but the flux exuding from the sample when doubled to 
account for the two sides of the specimen in the laboratory gives a good fit to the experimental data.

From the extracted diffusion species data on surface flux from the left side of thermseg/diffseg 1, the solid curve in Figure 10 is constructed where it is compared with the experimental data.

\section{Sample Problem No. 2 \\ Thermal Desorption Test of Beryllium}

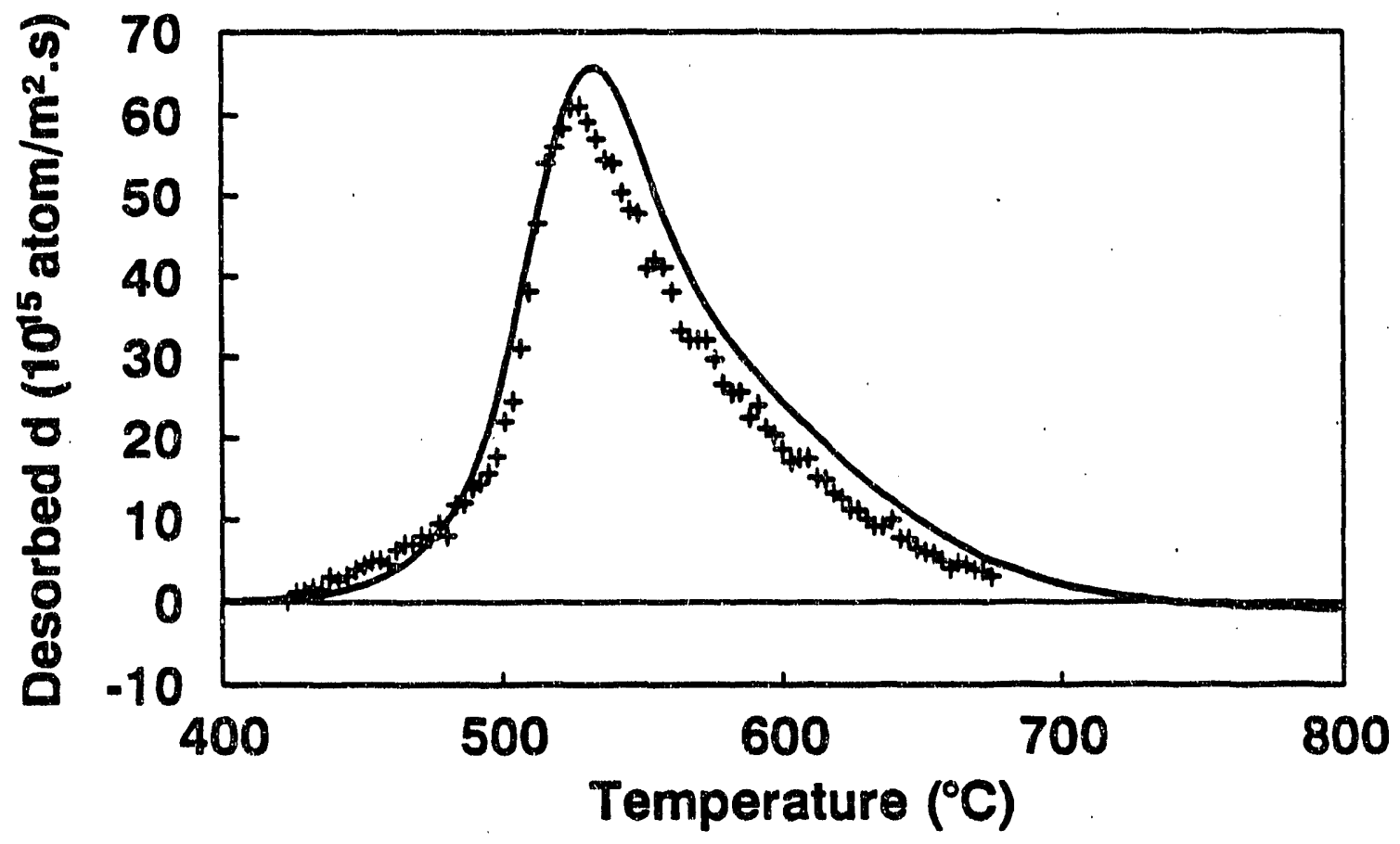

Figure 10. Comparison of calculated results (line) with experimental data $(+++)$ for thermal desorption test of beryllium after charging for 50 hours. 


\section{2c) Test Cell Release Experiment (val-2c.inp)}

This is a problem that involves multiple enclosures and chemical reactions. It is an experiment that was conducted at the Tritium Systems Test Assembly (TSTA) at Los Alamos National Laboratory and documented by Holland and Jalbert. ${ }^{12}$ The main part of the experiment was an exposure chamber with a nominal volume of $1 \mathrm{~m}^{3}$ which was lined with epoxy paint that is $0.16 \mathrm{~mm}$ thick. Tritium was admitted to the chamber as $\mathrm{T}_{2}$ at the commencement of the experiment. Normally moist (20\% R.H.) air was admitted to the chamber at the rate of $0.54 \mathrm{~m}^{3} / \mathrm{hr}$ constantly throughout the test. Samples of glycol taken from a bubbler just downstream from the exposure chamber were taken at intervals and scintillation counted to determine the time averaged HTO concentration in the chamber as a histogram in time. Tritium and water were absorbed into the paint during the initial part of the test and re-emitted later. Chemical reactions described by the formulae

$$
\begin{aligned}
& \mathrm{T}_{2}+\mathrm{H}_{2} \mathrm{O}=H \mathrm{H} \mathrm{O}+H T \\
& H T+\mathrm{H}_{2} \mathrm{O}=\mathrm{HTO}+\mathrm{H}_{2}
\end{aligned}
$$

took place within the exposure chamber, mainly as a consequence of the radioactivity of the tritium itself. Results from the measurement of the resulting HTO concentration in the exposure chamber following a $10 \mathrm{Ci}$ initial injection (effectively instantaneously) while purging with room air are shown in Figure 3 of the referenced article.

Modelling consists of three enclosures (1) the room from which air is drawn, (2) the exposure chamber, and (3) the tritium waste treatment system (TWT) to which the exhaust gases are directed. Only enclosure (2) is treated as "functional" or chemically active. The paint on the inside of the exposure chamber is treated as a diffusive segment with Henry's law solubility governing the concentration at its interface with enclosure (2) and non-flow conditions at the interface of the paint with the underlying aluminum foil. Experiments had previously demonstrated that there is virtually no transport of tritium into the aluminum foil. The technique for determining the constants and other 
information required to generate a model that gives reasonable results are given in the paper and are not duplicated here.

Data were extracted for the HTO concentration in the exposure chamber, enclosure 2. A solid curve representing these data is compared in Figure 11 with measurements made in bubblers in line with the exposure chamber exhaust. The period over which the bubblers were active in collecting HTO from the exposure chamber is shown on the time scale. They were integrated measurements over the intervals shown. The model fits best at extended times where the intercepts with the "average-value" line segments are at the correct times. Additional uptake and release channels for short times, beyond those modelled, may be responsible for the early-time disparity.

SAMPLE PROBLEM No. 3

HTO Concentration in TSTA Exposure Chamber

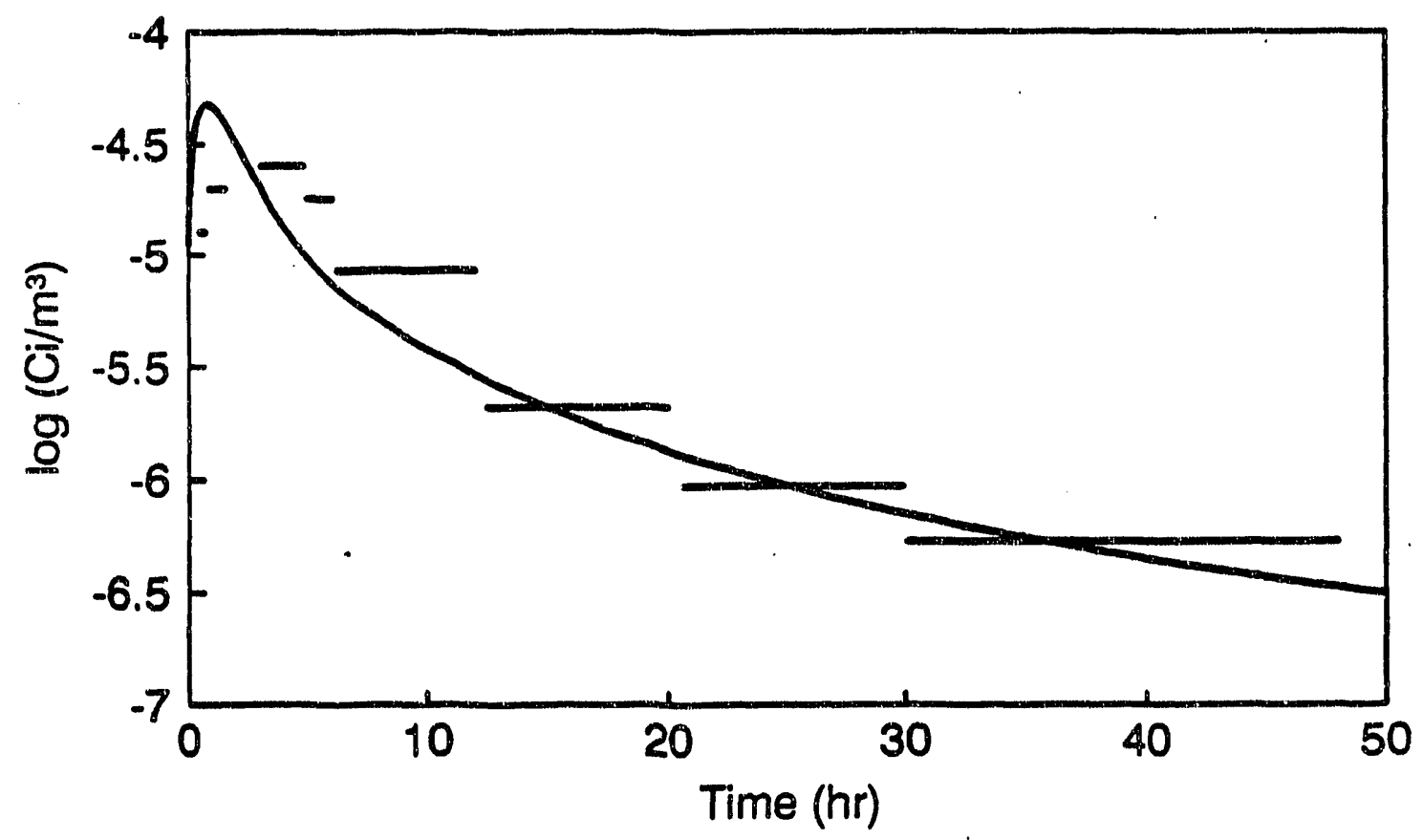

Figure 11. Comparison of TMAP4 calculational result with actual experimental data for the test cell release experiment. 


\section{INPUT FILES}

Input files are in two groups. One consists of the set of 37 files used in the verification coverage analysis. The second is the set of 14 files used in the validation process. All these files are listed on a diskette available with this report. Only the names of the first group, found in directory "verify" on the riskette are provided here. Files in the validation set are located in directory "valid" on the diskette. They are also listed here for convenience in evaluating the validation problems.

\section{Verification Files}

\begin{tabular}{|c|c|c|c|c|c|c|c|}
\hline BEO-PROB. INP & 3762 & $03-23-92$ & $7: 31 \mathrm{p}$ & GEN14. INP & 53951 & $03-05-92$ & $2: 55 p$ \\
\hline CHEMI . INP & 1516 & $03-23-92$ & $10: 06 p$ & GEN15. INP & 3769 & $05-14-92$ & $10: 57 a$ \\
\hline CHEM2 I INP. & 1997 & $03-23-92$ & $10: 17 p$ & GEN16. INP & 3890 & $05-14-92$ & $10: 58 a$ \\
\hline CLEANUP.INP & 3242 & $03-23-92$ & $7: 31 p$ & GENERAL. INP & 17803 & $08-07-91$ & $12: 54 p$ \\
\hline COMP.INP & 3593 & $12-10-90$ & $10: 34 a$ & HE.INP & 3343 & $03-23-92$ & $7: 32 p$ \\
\hline GENI. INP & 51304 & $03-05-92$ & $2: 05 p$ & MOD5-IN. INP & 23747 & $05-14-92$ & $11: 07 a$ \\
\hline GENIRST.INP & 481 & $05-14-92$ & $12: 14 p$ & PROB-1.INP & 2381 & $05-05-92$ & $6: 02 p$ \\
\hline GEN2. INP & 47015 & $03-05-92$ & $2: 19 p$ & PROB2CHG. INP & 3382 & $05-19-92$ & $11: 45 a$ \\
\hline GEN3. INP & 48607 & $03-05-92$ & $2: 20 p$ & PROB2HT. INP & 3660 & $05-19-92$ & $11: 43 a$ \\
\hline GEN4. INP & 52954 & $03-05-92$ & $2: 22 p$ & PROB-3. INP & 2446 & $05-21-92$ & $1: 29 p$ \\
\hline GEN5. INP & 52912 & $03-05-92$ & $2: 23 p$ & SHI.INP & 1458 & $05-14-92$ & $11: 39 a$ \\
\hline GEN6. INP & 1824 & $03-05-92$ & $2: 37 p$ & SH2.INP & 1369 & $05-14-92$ & $11: 41 a$ \\
\hline GEN7. INP & 1821 & $03-05-92$ & $2: 36 p$ & SH3. INP & 1301 & $05-14-92$ & $11: 42 a$ \\
\hline GEN8. INP & 1822 & $03-05-92$ & $2: 36 p$ & SH4. INP & 1519 & $05-14-92$ & $11: 45 a$ \\
\hline GEN9.INP & 1844 & $03-05-92$ & $2: 37 p$ & SH5. INP & 1851 & $05-14-92$ & $11: 45 a$ \\
\hline GEN10. INP & 1846 & $03-05-92$ & $2: 50 p$ & SH6:INP & 15865 & $03-05-92$ & $2: 01 p$ \\
\hline GENI1. INP & 1821 & $03-05-92$ & $2: 50 p$ & THERM1. INP & 1353 & $05-14-92$ & $11: 46 a$ \\
\hline GEN12. INP & 1814 & $03-05-92$ & $2: 50 p$ & THERM2. INP & 1346 & $05-14-92$ & $11: 48 a$ \\
\hline GEN13. INP & 1868 & $03-05-92$ & $2: 51 p$ & & & & \\
\hline
\end{tabular}




\section{Validation Files}

\section{VAL-1A. INP}

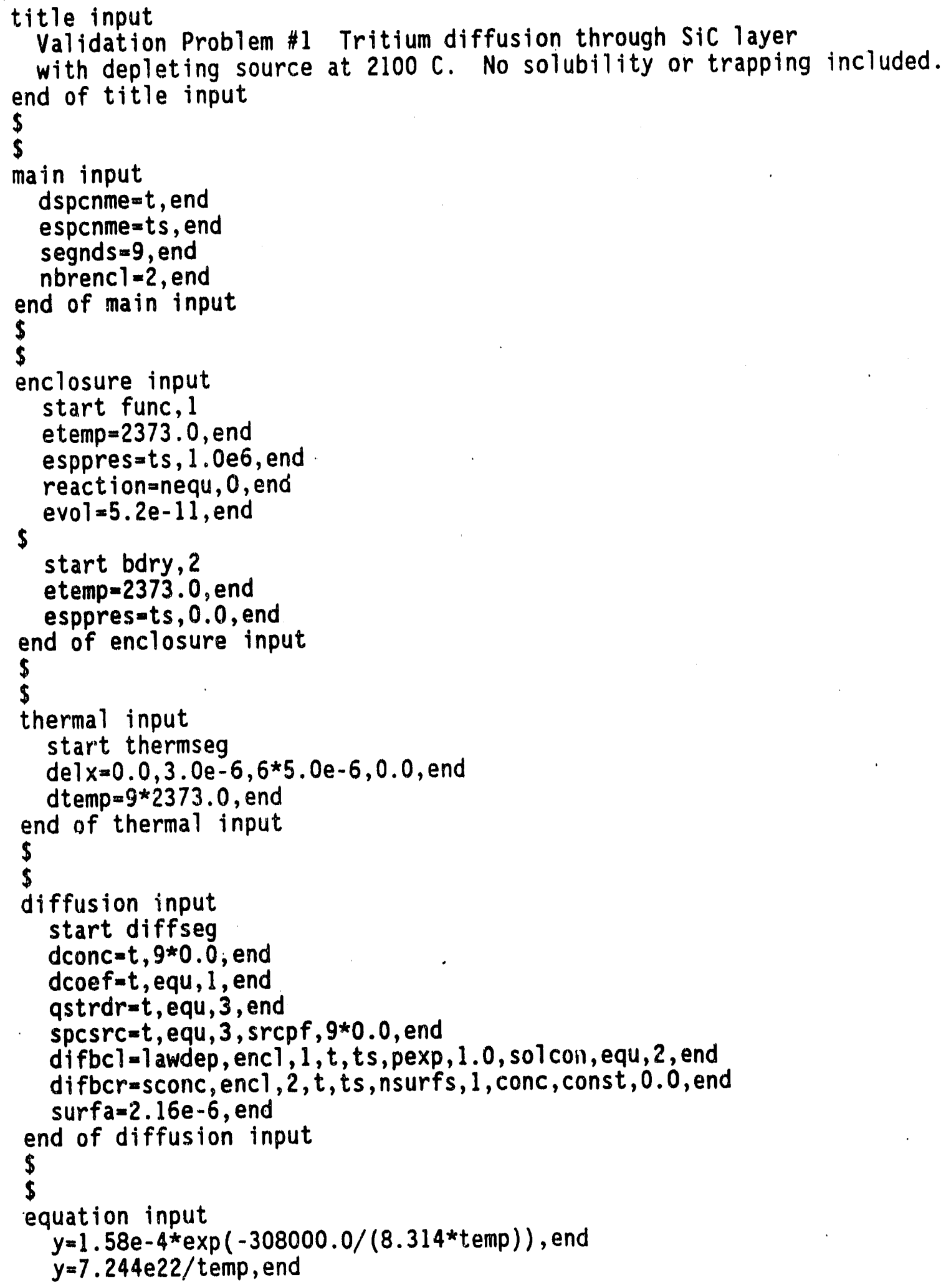




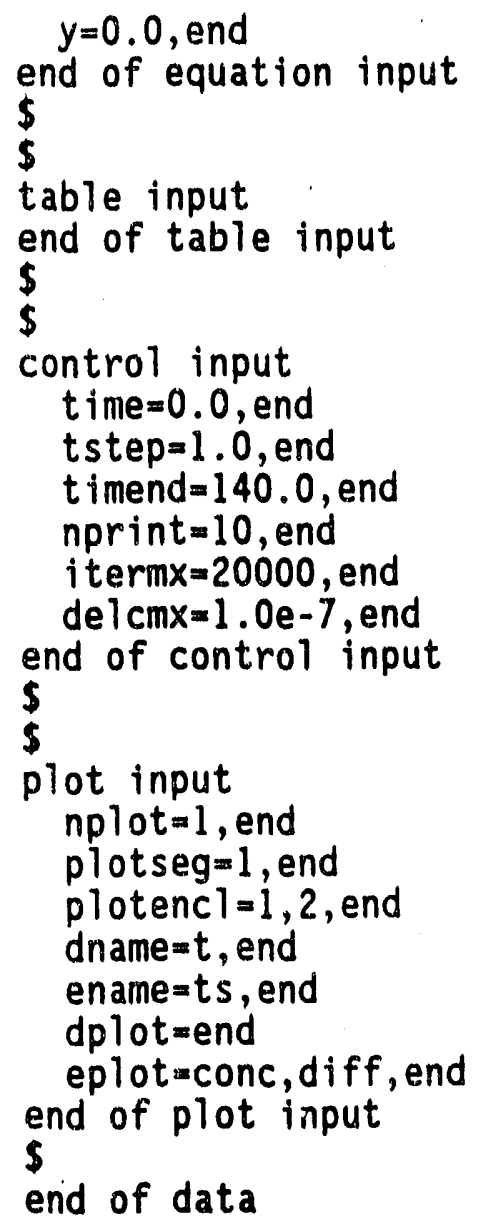

end of data 


\section{VAL-1B. INP}

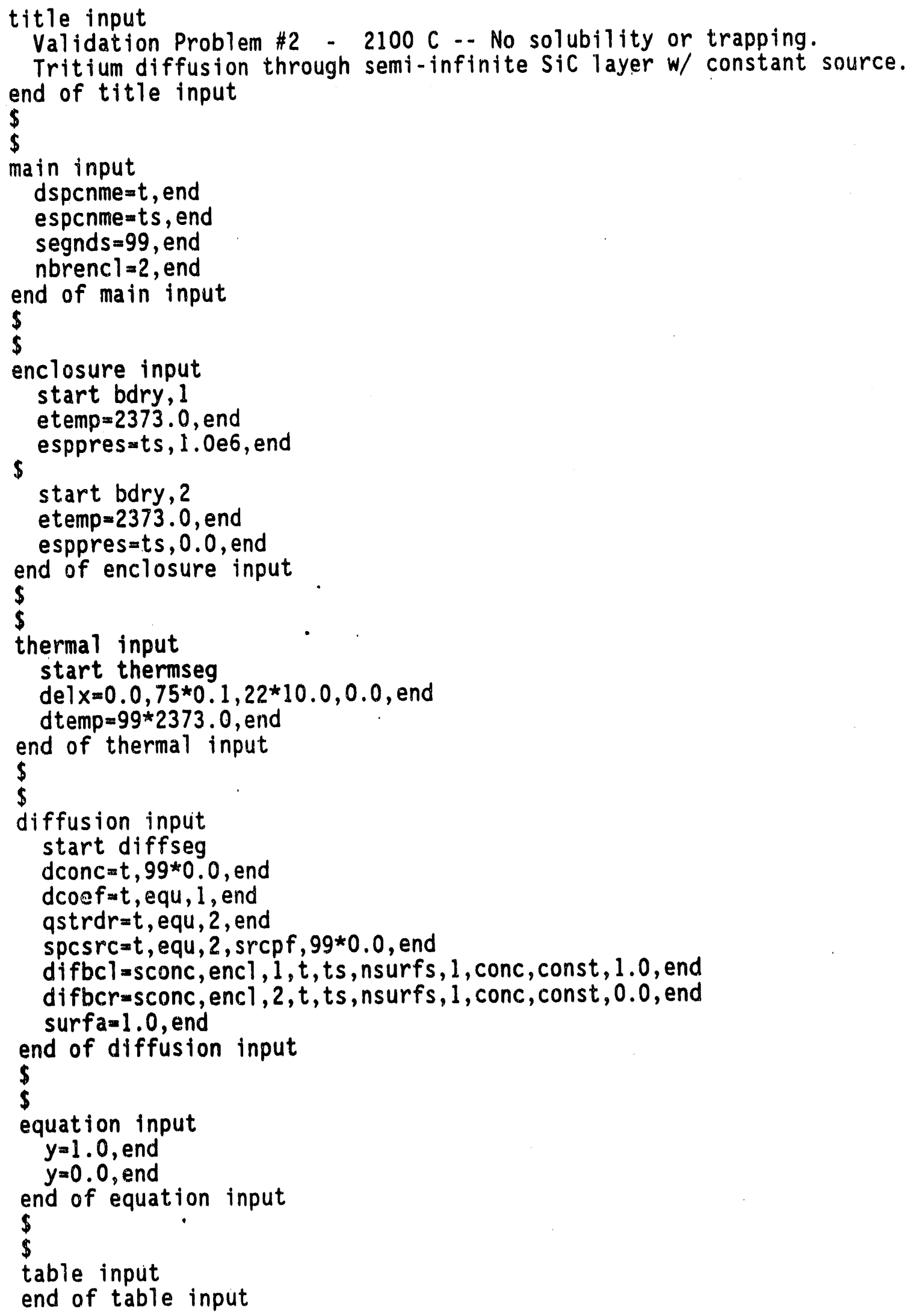




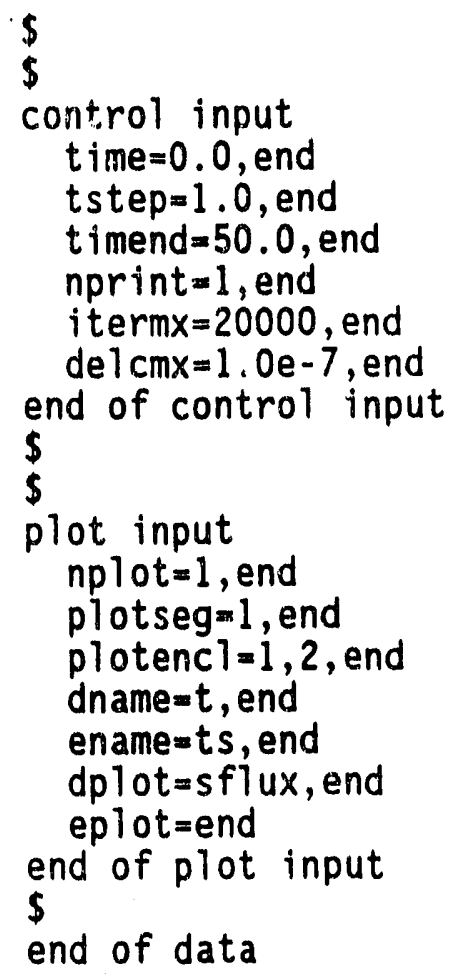




\section{VAL-1C. INP}

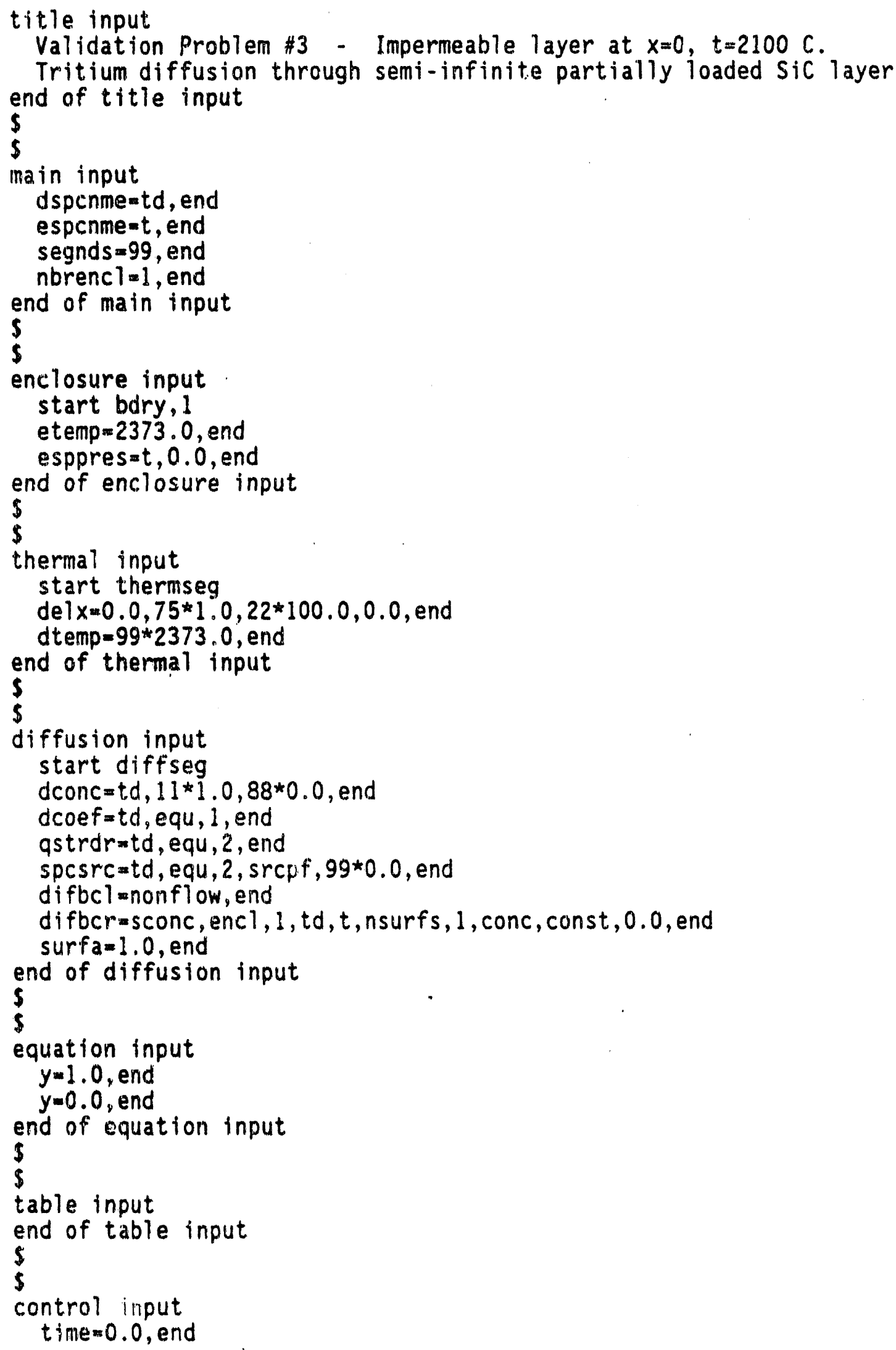




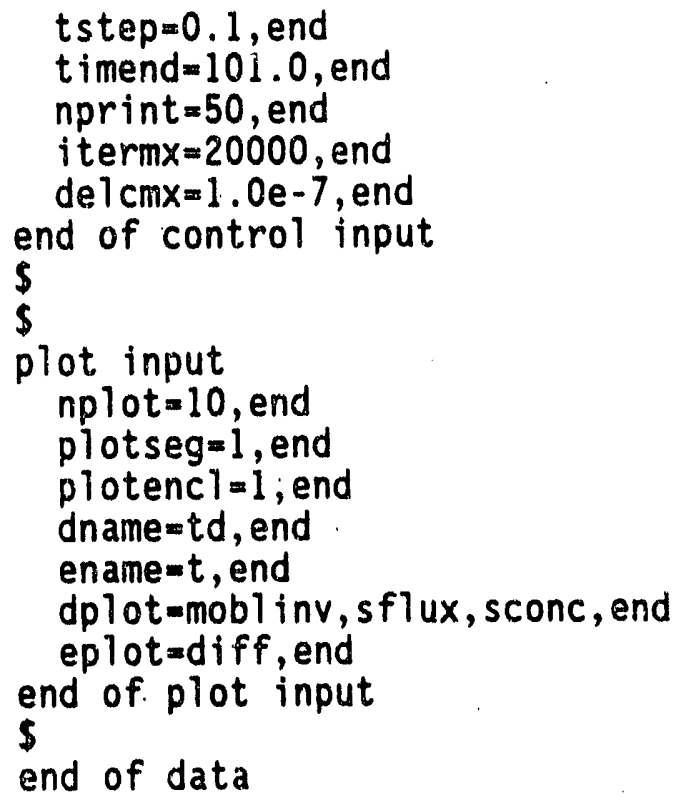


title input

Validation Problem \#4a - Trapping in a slab of constant upstream concentration - effective diffusivity limit end of title input

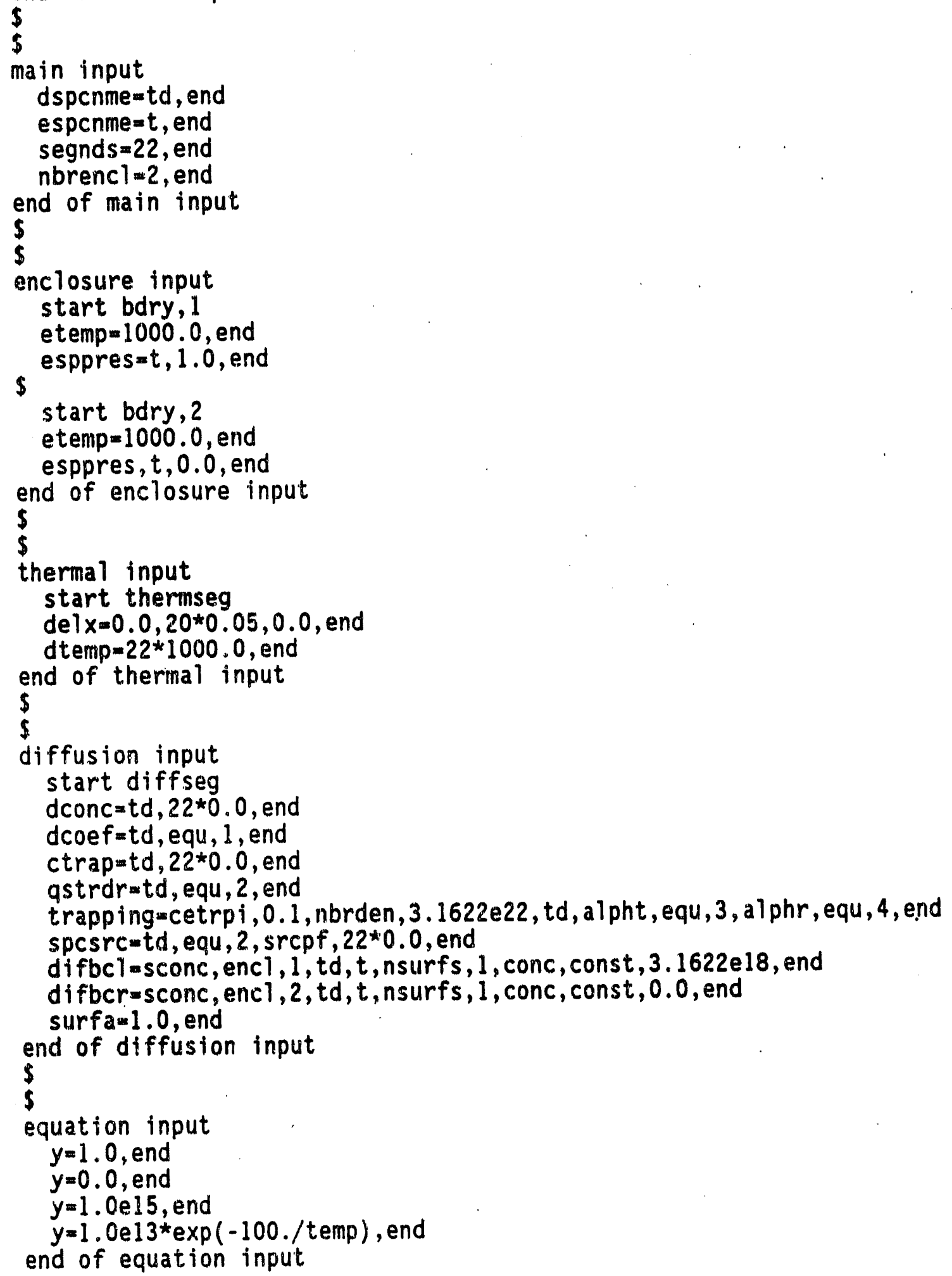




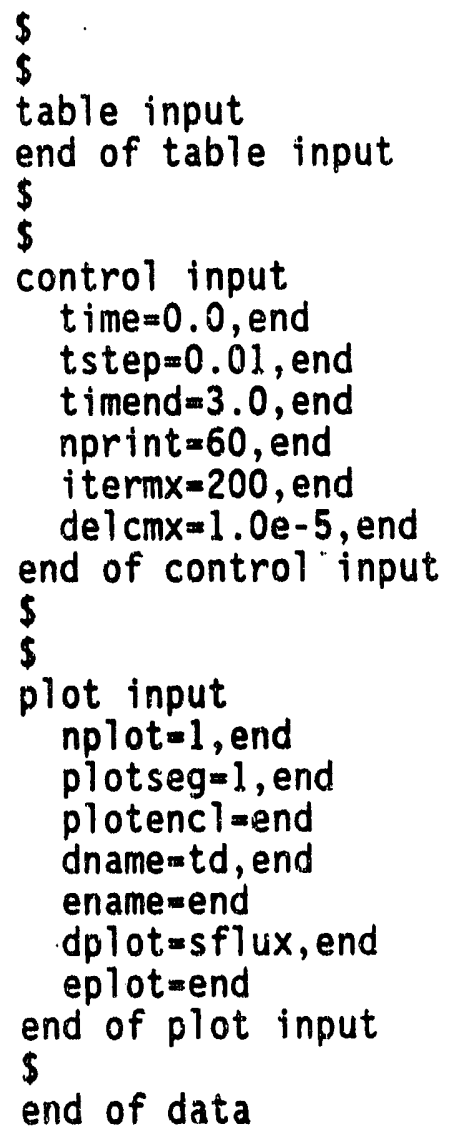




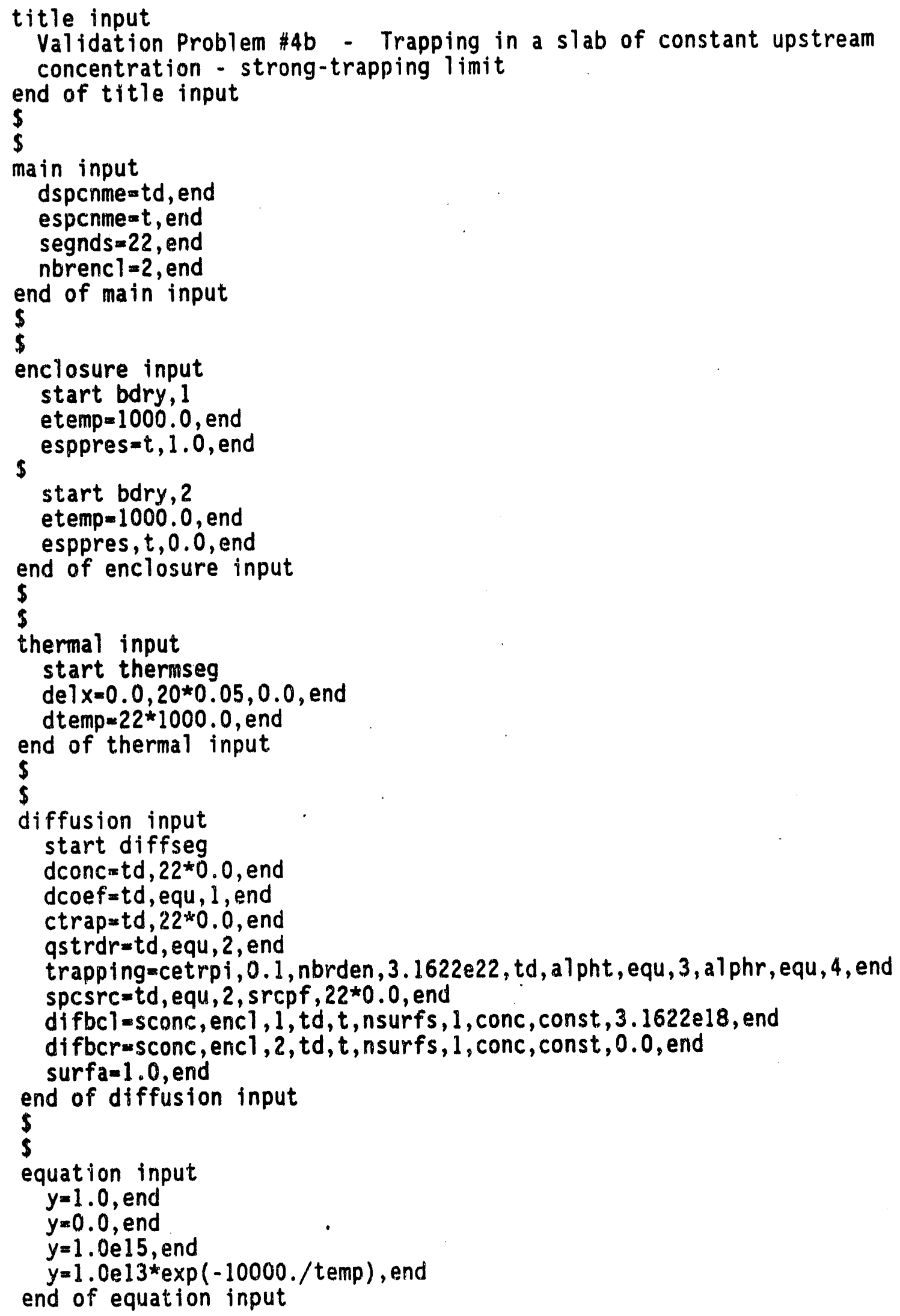




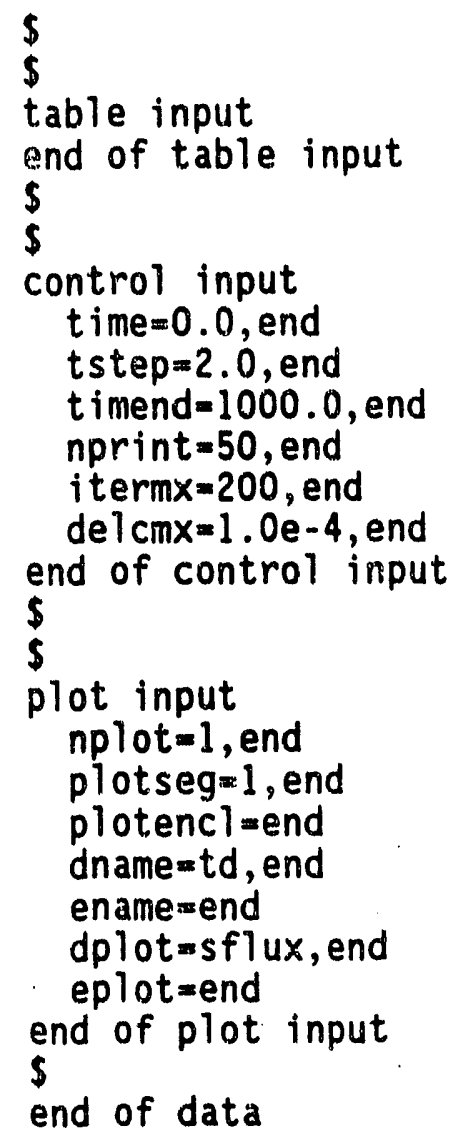




\section{VAL-1E. INP}

title input

Validation Problem \#5 - Tritium diffusion through PyC/SiC layer in NPR

fuel particles at $2100 \mathrm{C}$ with constant source and no solubility.

end of title input

s

$\$$

main input

dspcnme $=t d$, end

espcnme $=t$, end

segnds $=9,9$, end

nbrenc $7=2$, end

linksegs $=1,2$, end

end of main input

$\$$

$\$$

enclosure input

start bdry, 1

etemp $=2373.0$, end

$\$$

esppres $=t, 1.0 \mathrm{e} 6$, end

start bdry, 2

etemp $=2373.0$, end

esppres, $t, 0.0$, end

end of enclosure input

S

$\$$

thermal input

start thermseg

de $x=0.0,3.0 e-6,6 * 1.0 e-5,0.0$, end

$\$$

dtemp $=9 * 2373.0$, end

start thermseg

del $x=0.0,3.0 e-6,5.0 e-6,0.0,4 \star 6.25 e-6,0.0$, end

dtemp $=9 \star 2373,0$, end

end of thermal input

$\$$

$\$$

diffusion input

start diffseg

dconc $=t d, 9 * 0.0$, end

dcoef $=t d$, equ, 1 , end

qstrdr=td, equ, 3 , end

spcsrc $=t d$, equ, 3, srcpf, $9 * 0.0$, end

difbcl=sconc, encl $, 1, t d, t$, nsurfs, 1 , conc, const, $3.0537 \mathrm{e} 25$, end

di fbcr $=1$ ink, td, solcon, equ, 4, end

$\$$ surfa $=2.16 e-6$, end

start diffseg

dconc $=t d, 9 * 0.0$, end

dcoef $=t d$, equ, 2 , end

qstrdr $=t d$, equ, 3 , end

spcsrc $=t d, e q u, 3$, srcpf, $9 * 0.0$, end 
di fbcr $=$ sconc, enc $1,2, t d, t$, nsurfs, 1 , conc, const, 0.0 , end difbcl $=1$ ink, $t d$, solcon, equ, 4 , end

surfa $=2.16 e-6$, end

end of diffusion input

$\$$

$\$$

equation input

$y=1.0 e-1 * \exp (-64000.0 /(1.987 *$ temp $))$, end

$y=1.58 e-4 * \exp (-308000.0 /(8.314 *$ temp $))$, end

$y=0.0$, end

$y=1.0$, end

end of equation input

$\$$

$\$$

table input

end of table input

$\$$

control input

time $=0.0$, end

tstep $=1.0$, end

timend $=200.0$, end

nprint $=5$, end

iterm $x=20000$, end

de $7 \mathrm{~cm} x=1.0 \mathrm{e}-6$, end

end of control input

$\$$

$\$$

plot input

nplot $=1$, end

plotseg $=1$, end

plotenc $7=1,2$, end

dname $=t d$, end

en ame $=t$, end

dplot=moblinv, end

ep Tot=diff, end

end of plot input

$s$

end of data 


\section{VAL-1FA. INP}

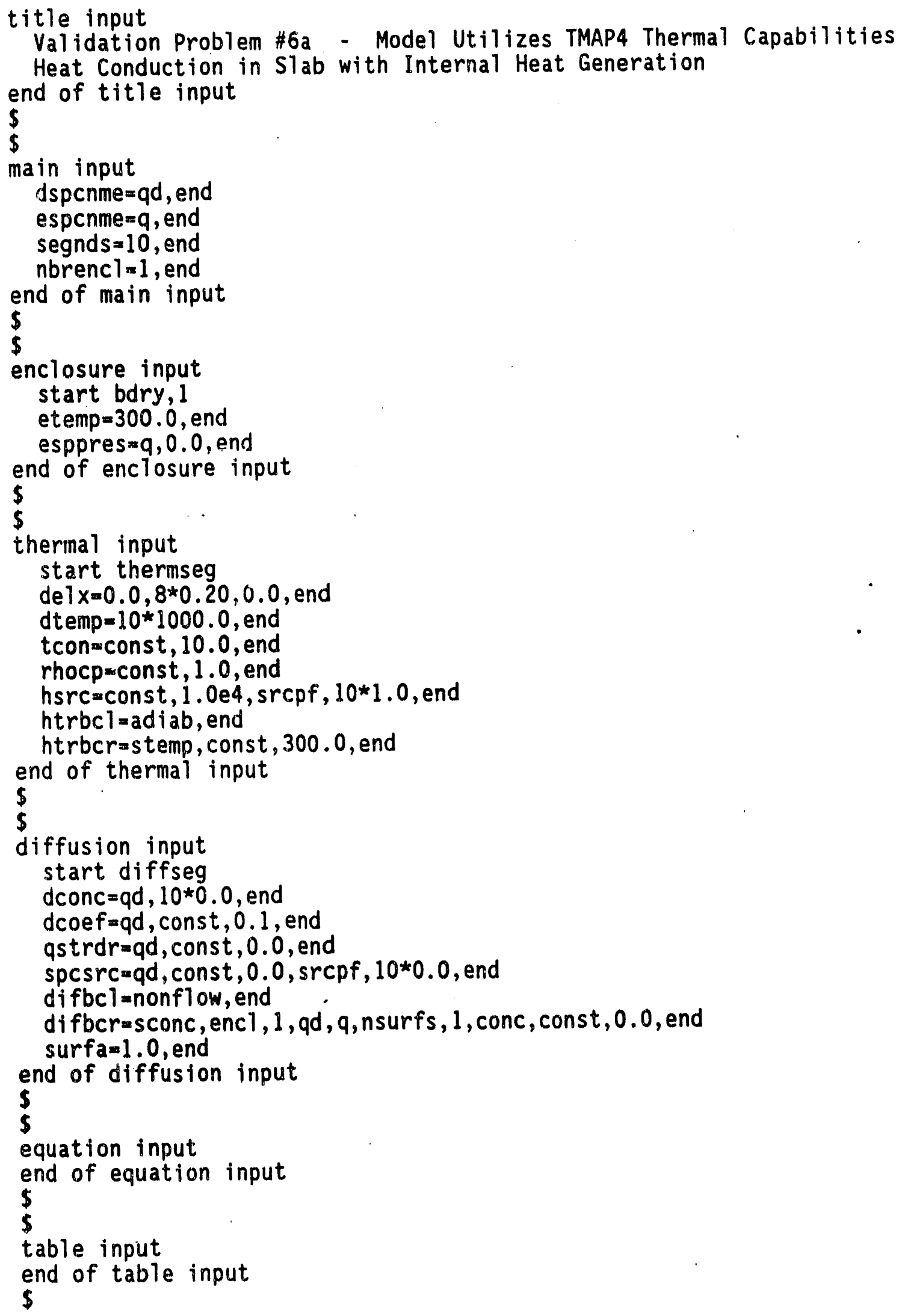




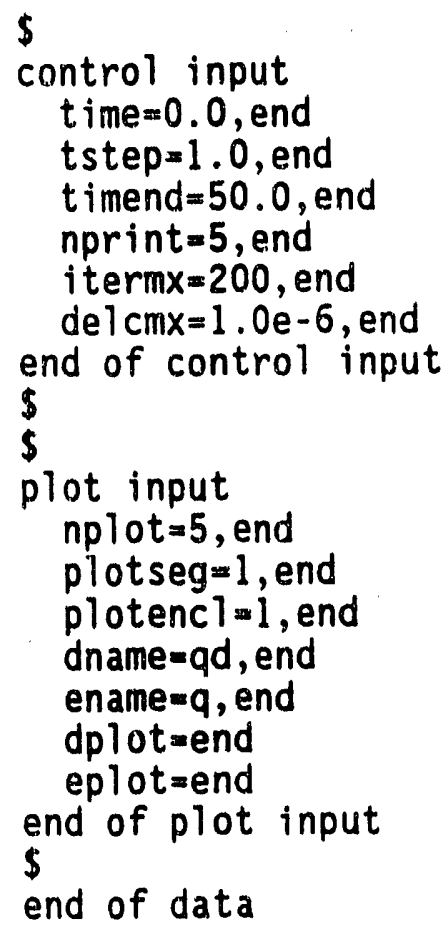




\section{VAL-1FB. INP}

title input

Validation Problem \#6b - Model Utilizes TMAP4 Thermal Capabilities

Prediction of Slab Temperature as a function of Time

end of title input

$\$$

main input

dspcnme $=t d$, end

espcnme $=t$, end

segnds $=18$, end

nbrencl $1=1$, end

end of main input

$\$$

$\$$

enclosure input

start biry, 1

etemp $=373.0$, end

esppres $=t, 0.0$, end

end of enclosure input

$\$$

$\$$

thermal input

start thermseg

de $7 x=0.0,1.25 e-2,14 * 2.5 e-2,1.25 e-2,0.0$, end

dtemp $=18 * 300.0$, end

end of thermal input

$\$$

$\$$

diffusion input

start diffseg

dconc $=$ td, $18 * 300.0$, end

dcoef $=t d$, const, $1.29035 e-3$, end

astrdr $=$ td, const, 0.0 , end

spcsrc $=$ td , const, 0.0 , srcpf, $18 * 0.0$, end

difbclanorrflow, end

difbcr $=$ sconc, enc $1,1, t d, t$, nsurfs, 1 , conc, const, 373.0 , end

surfa $=1.0$, end

end of diffusion input

$\$$

$\$$

equation input

end of equation input

$\$$

$\$$

table input

end of table input

$\$$

$\$$

control input

time $=0.0$, end

tstep $=10.0$, end

timend $=180.0$, end 


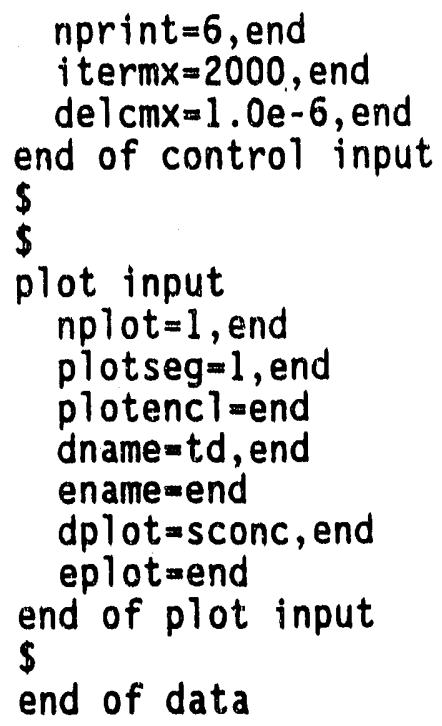




\section{VAL-1GA. INP}

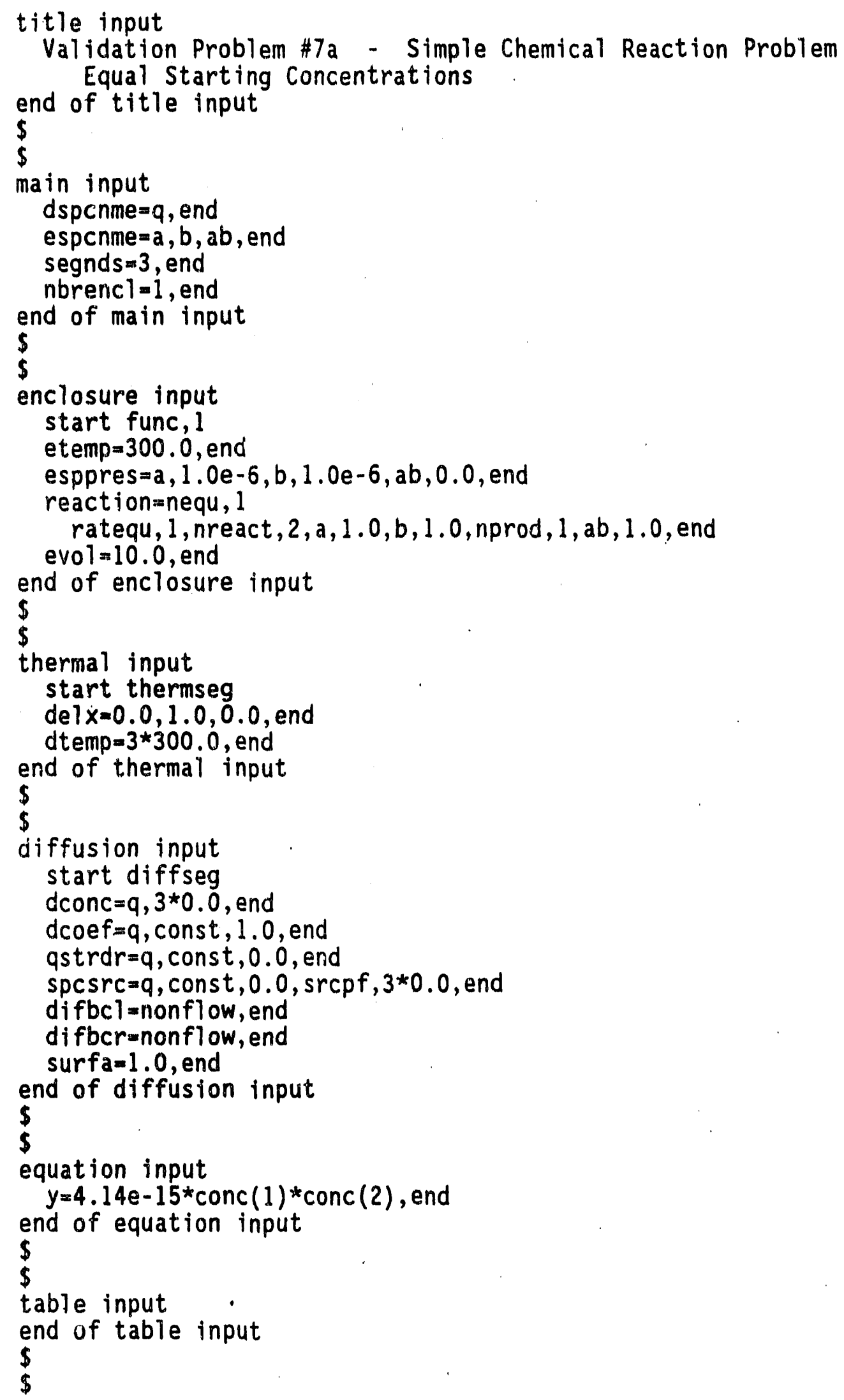




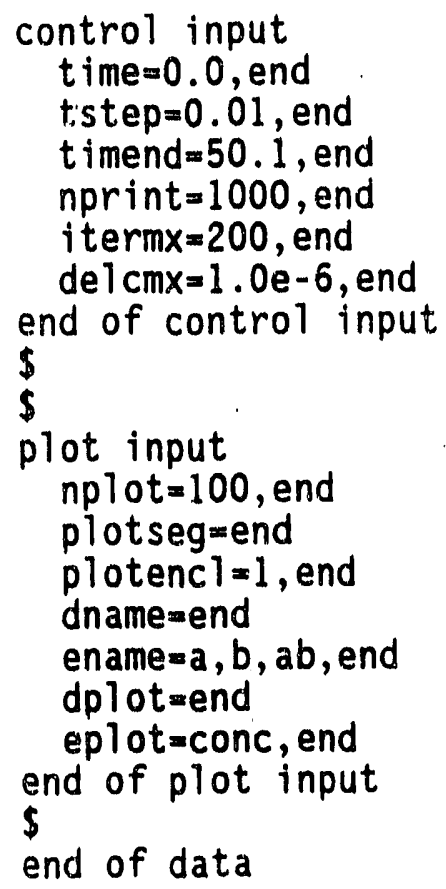




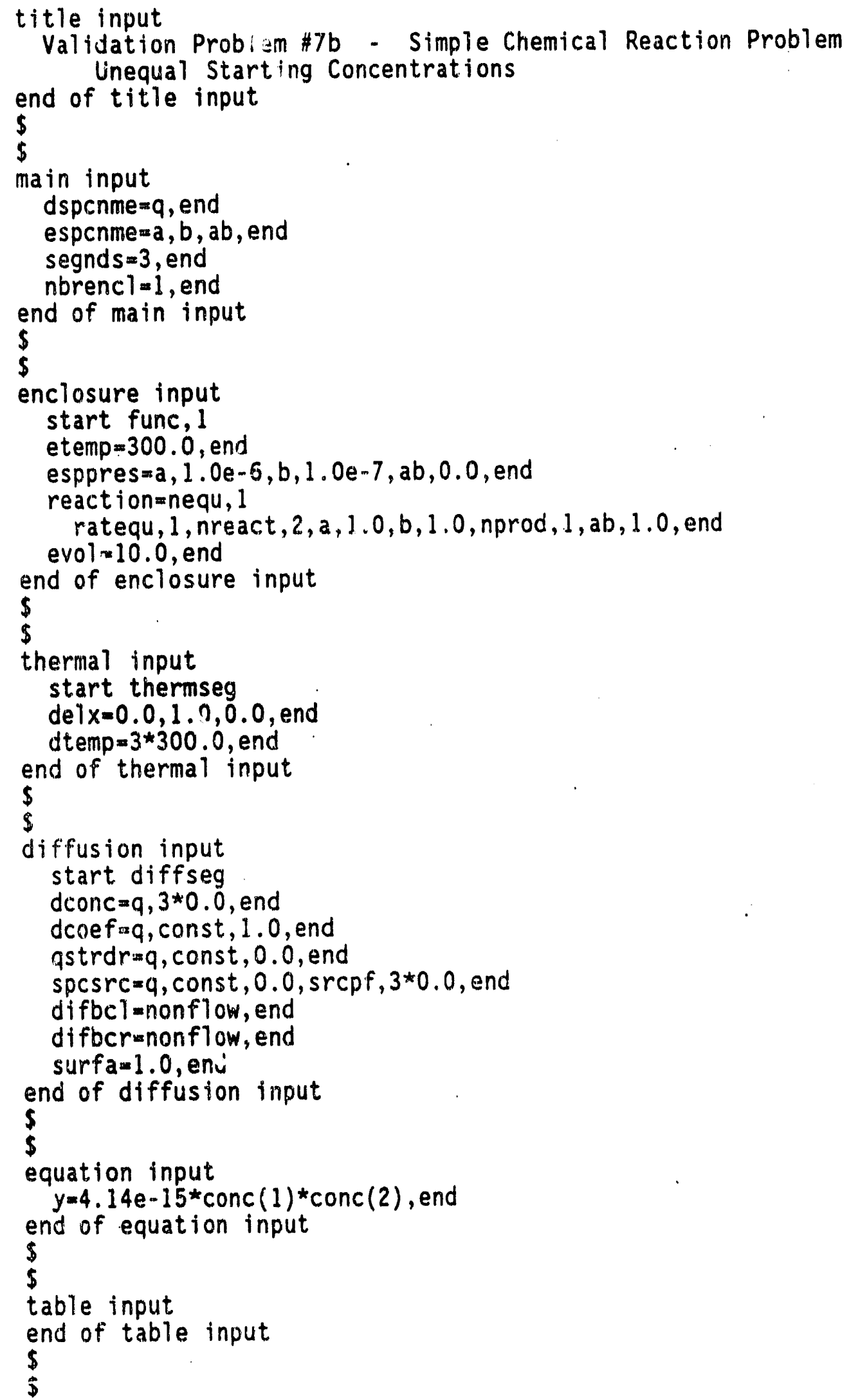




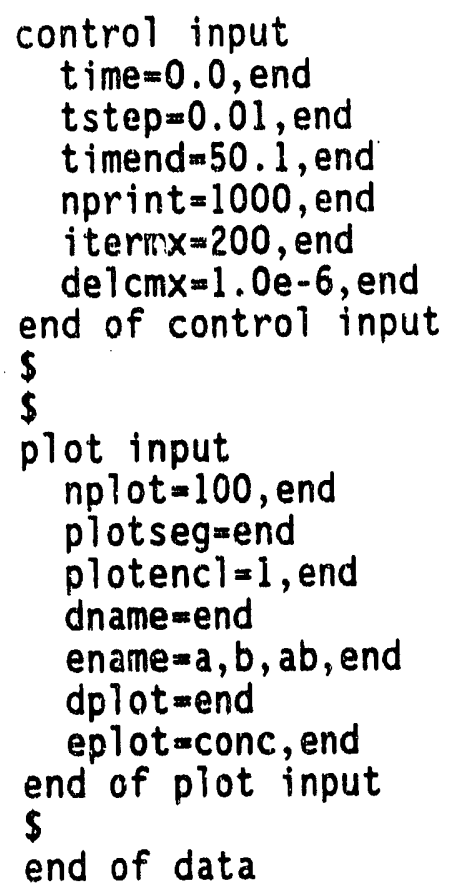


title input

TMAP Validation Problem \#8 - System (Multiple Enclosure Volumes) Problem end of title input

$\$$

main input

dspcnme $=t$, end

e spcnme $=$ t2, end

segnds $=3$, end

nbrencl $=3$, end

end of main input

$\$$

enclosure input

$\$$

start func, 2

etemp $=303$. , end

esppres $=t 2,0.0$, end

reaction $=$ nequ, 0 , end

evol = 1.0 , end

outflow $=$ nbrfiwp, 1 , qflow, const, 0.1 , rencl, 3 , end

start func, 3

etemp $=303$. , end

esppres $=t 2,0.0$, end

reaction = nequ, 0 , end

evol $=1.0$, end

outflow = nbrfiwp, 1 , qfiow, const, 0.1 , rencl, 1 , end

start bdry, 1

etemp $=303$., end

esppres $=t 2$, const, 1.0 , end

outflow = nbrflwp, 1 , qfiow, const, 0.1 , rencl, 2 , end

end of enclosure input

$\$$

thermal input

$\$$

start thermseg

del $x=0.0,1.0,0.0$, end

dtemp $=3 * 303.0$, end

end of thermal input

s

diffusion input

start diffseg

dconc $=t, 3 * 0.0$, end

dcoef $=t$, const, 1.0 , end

astrdr $=t$, const, 0.0 , end

spcsrc $=t$, const, 0.0 , srcpf, $3 * 0.0$, end

difbcl=nonflow, end

difber=nonflow, end

surfa $=1.0$, end

end of diffusion input

$\$$

equation input

end of equation input

$\$$ 
table input

end of table input

$\$$

control input

time $=0.0$, end

tstep $=0.01$, end

timend $=20.0$, end

nprint $=50$, end

iterm $x=20$, end

delcm $x=1 . e-6$, end

end of control input

$s$

plot input

nplot $=100$, end

plotseg=end

plotenc $1=2,3$, end

dname $=$ end

enamext2, end

dplot $=$ end

eplot=conc, conv, end

end of plot input

$\$$

end of data 


\section{VAL-2A. INP}

title input

SAMPLE PROBLEM No.1 - Plasma driven permeation of PCA end of title input

$\$$

main input

dspcnme $=d$, end

espcnme $=d 2$, end

segnds $=21$, end

nbrencl $=2$, end

end of main input

$\$$

enclosure input

S

start bdry, 1 , end

SImplantation side

etemp $=703$., end

esppres $=d 2$, tab 1,1 , end

start bdry, 2, end

\$Downstream side

etemp $=703$. , end

esppres $=\mathrm{d} 2$, const, $2 . e-6$, end

end of enclosure input

$\$$

thermal input

start thermseg, end

de $x=0.0,5 * 4.0 e-9,1.0 e-8,1.0 e-7,1.0 e-6,1.0 e-5,10 * 4.88 e-5,0.0$, end

dtemp $=21 * 703$. , end

end of thermal input

$\$$

diffusion input

start diffseg, end

dconc $=d, 21 * 0.0$, end

dcoef $=d$, const, $3.0 e-10$, end \$Diffusivity (m2/s)

qstrdr $=d$, const, 0.0 , end

spcsrc $=d$, tabl, 2 , srcpf $, 3 * 0.0,0.25,1.0,0.25,15 * 0.0$, end

di fbe $l=$ ratedep, enc $7,1, d$

d2, ksubd, equ, l, d, ksubr, equ, 2 , end

di fbcr $=$ ratedep, enc $7,2, d$ $\mathrm{d} 2$, ksubd, const, $1.7918 \mathrm{e} 15, \mathrm{~d}, \mathrm{ksubr}$, const, $2.0 \mathrm{e}-31$, end

surfa=1.0, end

SNo temperature gradient

end of diffusion input

$\$$

equation input

$\$$ Dissociation constant (d $\left.2 / m^{\wedge} 2 . S . P a^{\wedge} 1 / 2\right)$

$y=8.959 \mathrm{e} 18 *(1.0-0.9999 * \exp (-6.0 \mathrm{e}-5 *$ time $))$, end SEq.1

$\$$ Recombination constant ( $\left.m^{\wedge} 4 / d_{2} 2 . s\right)$

$y=1.0 \mathrm{e}-27 *(1.0-0.9999 * \exp (-6.0 \mathrm{e}-5 *$ time $))$, end $\$ E q .2$

end of equation input

$\$$

table input

$\$$ Upstream enclosure pressure history

$0.0,4.0 e-5,6420.0,4.0 e-5,6420: 1,9.0 e-6,9420.0,9.0 e-6,9420.1,4.0 e-5$

$12480.0,4.0 \mathrm{e}-5,12480.1,9.0 \mathrm{e}-6,14940.0,1.9 \mathrm{e}-6,14940.1,4.0 \mathrm{e}-5,18180.0$

$4.0 e-5,18180.1,9.0 e-6,1.0 e 10,9.0 e-6$, end

\$Table 1 
\$ Implantation fiux (d/m2s)

$0.0,4.9 \mathrm{e} 19,6420.0,4.9 \mathrm{e} 19,6420.1,0.0,9420.0,0.0,9420.1,4.9 \mathrm{e} 19$

$12480.0,4.9 \mathrm{e} 19,12480.1,0.0,14940.0,0.0,14940.1,4.9 \mathrm{e} 19,18180.0$

$4.9 \mathrm{e} 19,18180.1,0.0,1.0 \mathrm{e} 10,0.0$, end

sTable 2

end of table input

$\$$

control input

time $=0.0$, and

tstep $=20.0$, end

timend $=1980$, and

nprint $=60$, and

itermx $=90$, end

de $1 c m x=1 . e-8$, end

end of control input

$\$$

plot input

nplot $=3$, end

plotseg $=1$, end

\$ Plot points every minute

plotenc $l=1,2$, end

dname $=d$, end

ename $=\mathrm{d} 2$, end

dplot=moblinv, sflux, end

eplot $=$ end

end of plot input

end of data 
title input

R. G. Macaulay-Newcombe's thermal charging problem for gas absorption into a wafer of polished beryllium with a thin oxide film.

end of title input

$\$$

main input

dspcnine $=d$, end

espcnme $=\mathrm{d} 2$, end

segnds $=20,17$, end

nbrencl $=1$, end

linksegs $=1,2$, end

end of main input

$\$$

enclosure input

$\$$

start bdry, 1 , end

etemp $=773.00$, end

esppres $=\mathrm{d} 2$, equ, 6 , end

end of enclosure input

$\$$ 0xide has 20, Be has 17

$\$$

thermal input

S Segment 1 - BeO film

start thermseg, end

del $x=0.0 ; 18 * 1.0 e-9,0.0$, end

dtemp $=20 * 773.0$, end

tcon $=$ const, 159.2 , end

rhocp=const $3.0 \mathrm{e} 6$, end

hsrc $=$ const , 0.0 , srcpf , $20 * 0.0$, end

htrbcl = stemp, equ, 1 , end

htrbcr $=1$ ink, end

hgap $=$ const, $1 . e 6$, end

\$ Segment 2 - Be metal - half thick

start thermseg, end

de $7 x=0.0,1.0 e-9,1 . e-8,1 . e-7,1 . e-6,1 . e-5,10 * 1.888 e-5,0.0$, end

dtemp $=17 * 773.0$, end

tcon $=$ const, 168.0 , end

rhocp $=$ const, $3.37 e 6$, end

hsrc $=$ const, 0.0 , srcpf, $17 * 0.0$, end

htrbclimink, end

htrbcr $=$ adiab, end

end of thermal input

\$

diffusion input

$\$$ Seg No. 1 BeO film

start diffseg, end

$\mathrm{dconc}=\mathrm{d}, 20 * 0.0$, end

$d c o e f=d$, equ, 2 , end

qstrdr $=d$, const, 0.0 , end

spcsrc $=d$, const, 0.0 , srcpf, $20 * 0.0$, end

\$ Outside of sample

\$ Pressure history in Eq. 6

$\$$ The oxide and Be are joined

difbcl $=1$ awdep, encl 1

d

$d 2$, pexp, 0.5 , solcon, equ, 3 , end

$\$ D$ for $d$ in $B e O$ in $E q .2$

$\$ Q^{*}$ of $n e$ ronsequence 
Eq.3

difbcr $=1$ ink, $d$, solcon, equ, 3 , end

\$ Solubility for $D$ in $B e O$ in

surfa $=1.04 \mathrm{e}-4$, end

$\$$ Seg No. 2 Be foil - half thickness

start diffseg, end

dconc $=d, 17 * 0.0$, end

dcoef $=d$, equ, 4 , end

qstrdr $=d$, const, 0.0 , end

spcsrc $=d$, const, 0.0 , srcpf, $17 * 0.0$, end

Eq. 5 difbcl $=1$ ink, $d$, solcon, equ, 5 , end

difber=nonflow, end

surfa $=1.04 e-4$, end

end of diffusion input

$\$$

equation input

$\$$ Temperature history equation

$y=773$. - int (time/180000.)*(1-exp(-(time-180000.)/2700.) )*475., end \$Eq.1 Temp

$\$$ Diffusion and solubility equations

$y=1.40 \mathrm{e}-4 * \exp (-24408$. $/$ temp $)$, end

$y=5.00 \mathrm{e} 20 * \exp (9377.7 /$ temp $)$, end

$y=8.0 e-9 * \exp (-4220 . /$ temp $)$, end

$\mathrm{Be}-2)$.

$y=7.156 \mathrm{e} 27 * \exp (-11606 . /$ temp), end

* Pressure history equation $13300.0 *(1-$ int (time/180015.) $)+1.0 e-6$, end. \$Eq. 6 Pressure history

(a) f equation input

$\$$

table input

end of table input

$\$$

control input

time $=0.0$, end

tstep $=60.0$, end

timend $=182400$. , end

nprint $=300$, end

iterm $x=90$, end

del $c m x=1 . e-8$, end

end of control input

$s$

plot input

nplot $=10$, end

plotseg=1,2, end

\$Eq.2 $D$ of $d$ in BeO (Fowler 1)

SEq.3 $S$ for $d$ in $\mathrm{BeO}$

$\$ D$ for $d$ in Be in Eq. 4

$\$ Q^{*}$ of no consequence

Solubility for $D$ in Be in

$\$$ Midplane of foil - no flow

SEq.4 0 of $d$ in Be (Abramov

\$Eq.5 $S$ for $d$ in Be (Swarisiger)

plotencl=end

dname $=d$, end

ename=end

dplot=mobl inv, sflux, end

eplot=end

end of plot input

end of data

$\$$ Cycles

$\$$ Seconds

$\$$ One minute time step

$\$ 50 \mathrm{hr}+45 \mathrm{~min}$ cooling

$\$$ Output at 5-hr intervals 


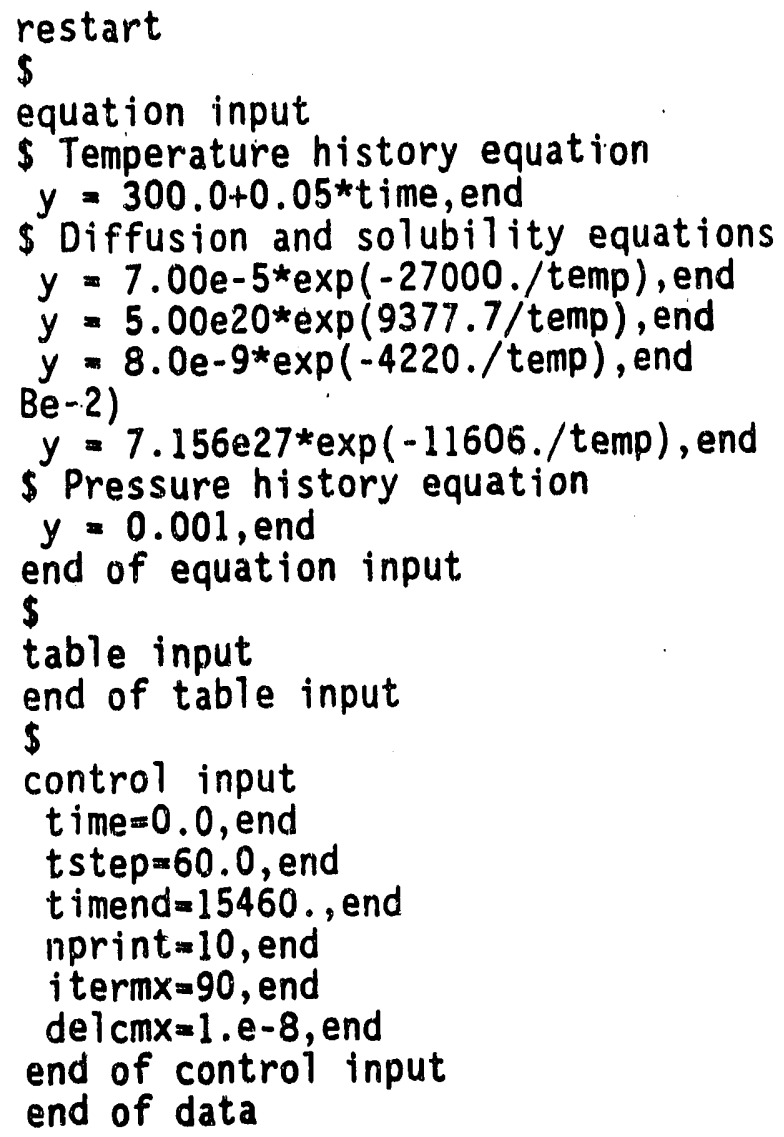

SEq. 1 Heat rate $3 \mathrm{~K} / \mathrm{min}$

\$Eq.2 $D$ of $d$ in BeO (Fowler 2)

$\$ E q .3 S$ for $d$ in $B e O$

SEq.4 $D$ of $d$ in Be (Abramov

SEq.5 $S$ for $d$ in Be (Swansiger)

\$Eq. 6 Pressure history
\$ One minute sampling
$\$$ Time to $800 \mathrm{C}$
$\$$ Cyciles 


\section{VAL-1C.INP}

title input

SAMPLE PROBLEM 3 - HTO history in an exposure chamber at TSTA end of title input

$\$$

main input

dspcnme $=t 2 d$, htd, htod, h2od, end

espcnme $=$ t2, ht , hto, h2o, end

segnds $=12$, end

nbrencl $=3$, end

end of main input

S

enclosure input

start func, 2 , end

etemp $=303$, , end

esppres $=t 2,0.434, \mathrm{ht}, 0$. , hto $0 ., \mathrm{h} 20,714$. , end

outflaw=nbrfiwp, 1, qflow, const, $1.5 e-4$, rencl, 3 , end

reaction $=$ nequ, 2 , ratequ, 1

nreact $, 2, \mathrm{t2}, 1$., h2o, 1., nprod, 2, hto, 1 . , ht , 1 .

ratequ, 2

nreact, 2, ht , 1., h20,1., nprod, 1, hto , 1., end

evol $=0.96$, end

start bdry, 1 , end

$\$$ Source air from room

etemp=303., end

esppres $=t 2,0$. , ht, 0 ., hto 0 . , h20,714., end

outflowanbrfiwp, 1 , qflow, const, $1.5 e-5$, rencl, 2 , end \$Low by $10 \times$

start bdry, 3 , end

s Sink, TWT system

etemp=303., end

esppres $=$ t2, 0 . , ht, 0 . hto 0 . , h20,714., end

end of enclosure input

$\$$

thermal input

start thermseg

del $x=0 ., 10 * 1.6 e-5,0 .$, end

\$ Paint inside the exposure chamber

dtemp $=12 * 303$. , end

end of thermal input

$\$$

diffusion input

start diffseg

dconc $=$ t $2 d, 12 * 0$. , htd , 12*0., htod, 12*0., h2od, 12*0., end

dcoef $=t 2 d$, const, $4 . e-12$, htd, const, $4 . e-12$

htod, const, 1.e-14, h2od, const, 1.e-14, end

qstrdr $=t 2 d$, const, 0 ., htd, const, 0 . , htod, const, 0 ., h2od, const, J. end

spcsrc $=t 2 d$, const, 0 ., srcpf, $12 * 0$. , htd, const, 0 . , srcpf, $12 * 0$.

htod, const, 0 ., srcpf, $12 * 0$., h2od, const, 0 ., srcpf, $12 * 0$. , end

difbcl $=1$ awdep, enc $1,2, t 2 d, t 2$, pexp, 1., solcon, const, 4. el 19

htd, ht, pexp, 1., solcon, const, 4.e19

htod, hto, pexp, 1., solcon, const, 6.e19

difbcr=nonflow, end

h2od, h20: pexp, 1., solcon, const, 6. e24, end

surfa $=5.6$, end

end of diffusion input

$\$$ 
equation input

$\$$ Reaction rate equations

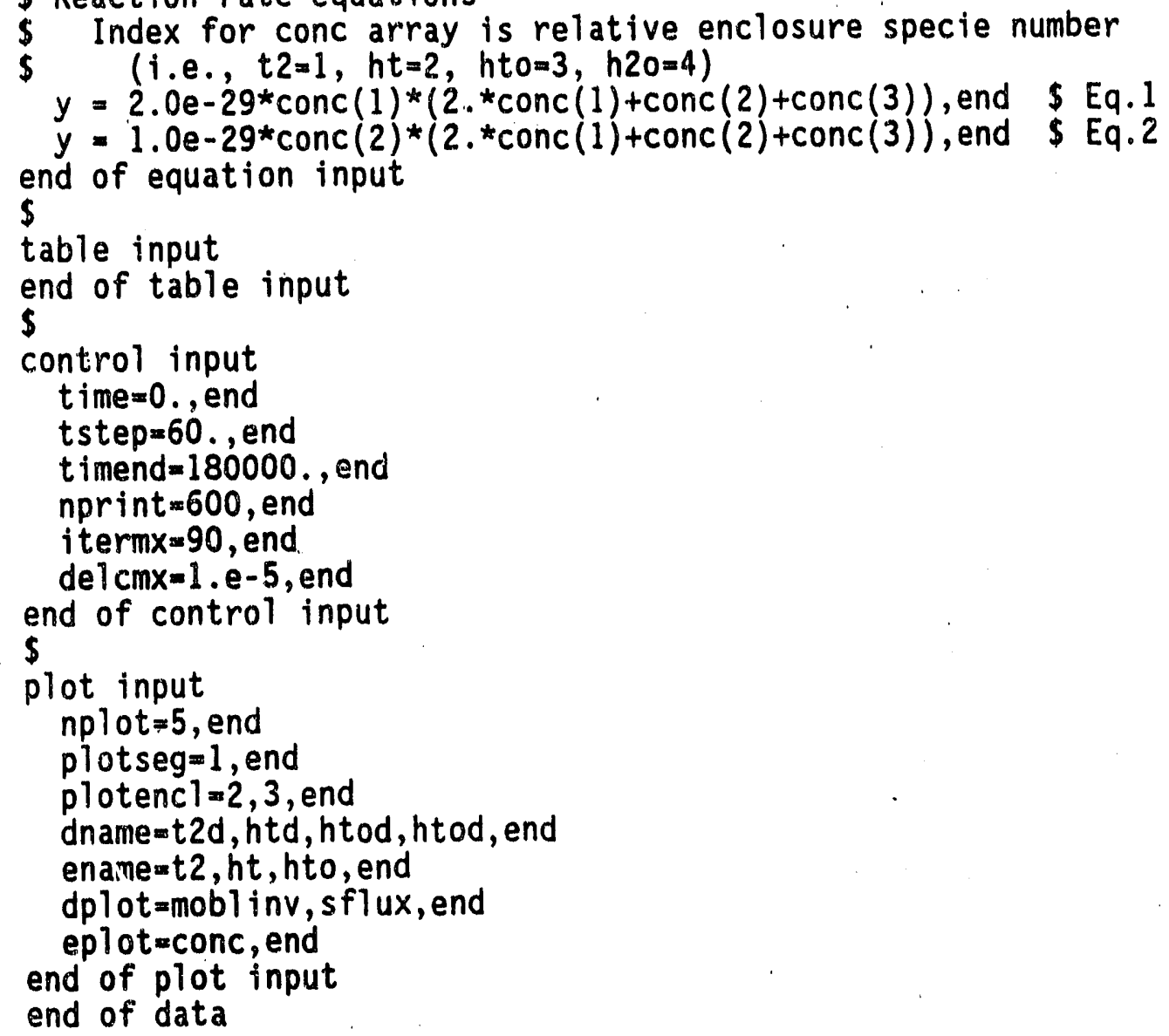




\section{REFERENCES}

1. G. R. Longhurst, D. F. Holland, J. L. Jones, and B. J. Merrill, "TMAP4 User's Manual," EGG-FSP-10315, June 12, 1992, Idaho National Engineering Laboratory, Idaho Falls, ID 83415.

2. S. L. Harms, "Comparison of TMAP Results with Selected Analytical Solutions," EDF No. NPR-MHTGR-0265, July 9, 1991, EG\&G Idaho, Inc., Idaho Falls, ID 83415.

3. G. R. Longhurst, "Trapping Effects on diffusion Transients," EGG-FSP-9789, July 1991, Idaho National Engineering Laboratory, Idaho Falls, ID 83415.

4. R. A. Anderl, D. F. Holland, D. A. Struttmann, G. R. Longhurst, and B. J. Merrill, "Tritium Permeation in Stainless Steel Structures Exposed to Plasma Ions," Proceedings, Eleventh Symposium on Fusion Engineering, November 18-22, 1985, Austin, TX, Vol I, pp. 644-649, IEEE Cat. No. CH2251-7.

5. R. G. Macaulay-Newcombe, D. A. Thompson, and W. W. Smeltzer, "Deuterium Diffusion, Trapping and Release in Ion-implanted Beryllium," Fusion Engineering and Design 8 (1991) 419.

6. K. L. Wilson, et al., "Beryllium--a better tokamak plasma-facing material?" Journal of Vacuum Science and Technology A 8 (3) (May/June-1990) 1750.

7. E. Abramov, et al., "Deuterium Permeation and Diffusion in High Purity Beryllium," CFFTP.G-9013, May 1990.

8. G. R. Longhurst, "Tritium Behavior in ITER Beryllium," EGG-FSP-9304, October 1990.

9. R. A. Causey, et al., "Tritium retention and migration in beryllium," Journal of Nuclear Materials, 176 \& 177 (1990) 654.

10. R. G. Macaulay-Newcombe, et al., "Thermal Absorption and Desorption of Deuterium in Beryllium and Beryllium Oxide," Fifth International Conference on Fusion Reactor Materials, Clearwater, FL, November 20-24, 1991.

11. J. D. Fowler, et al., "Tritium Diffusion in $\mathrm{Al}_{2} \mathrm{O}_{3}$ and $\mathrm{BeO}$," Journal of the American Ceramic Society, 60 (3-4) (Mar/Apr 1977) 155.

12. D. F. Holland and R. A. Jalbert, "A Model for Tritium Concentration Following Tritium Release into a Test Cell and Subsequent Operation of an Atmospheric Cleanup System," Proceedings, Eleventh Symposium on Fusion Engineering, November 18-22, 1985, Austin, TX, Vol I, pp. 638-643, IEEE Car. No. CH2251-7. 

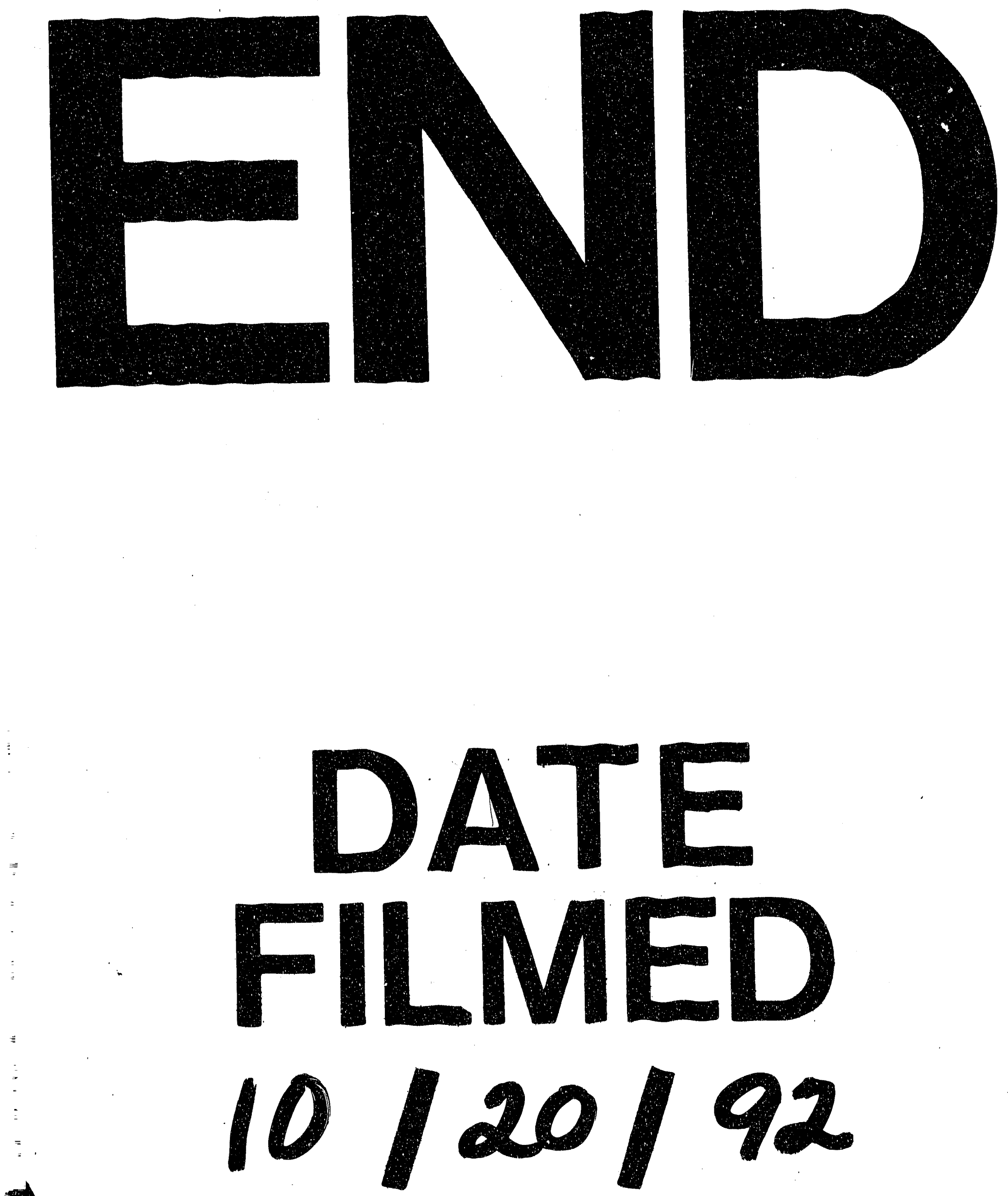

7 
